\title{
Highly Chemo- and Regioselective Construction of Spirocarbocycles by a Pd(0)-Catalyzed Dearomatization of Phenol-Based Biaryls with 1,3- Dienes
}

\author{
Lei Luo, ${ }^{\dagger}$ Huayu Zheng, ${ }^{\dagger}$ Jingjing Liu,${ }^{\dagger}$ Hui Wang, ${ }^{\dagger}$ Yaoyu Wang, ${ }^{\dagger}$ and Xinjun Luan $*^{\dagger, \ddagger}$ \\ ${ }^{\dagger}$ Key Laboratory of Synthetic and Natural Functional Molecule Chemistry of the Ministry of \\ Education, College of Chemistry \& Materials Science, Northwest University, Xi'an 710127, China \\ ${ }^{\ddagger}$ State Key Laboratory of Elemento-organic Chemistry, Nankai University, Tianjin 300071, China
}

Email: xluan@nwu.edu.cn

\section{Supporting Information}

Table of Contents:

A. General information:

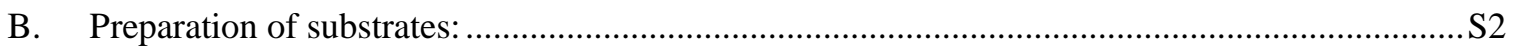

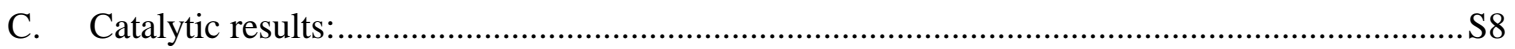

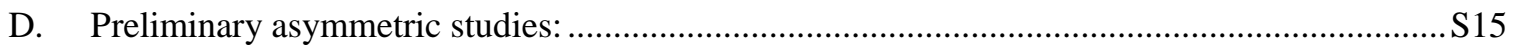

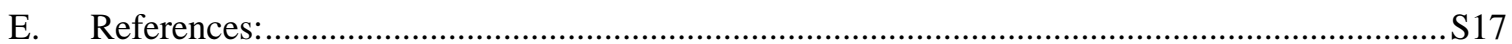

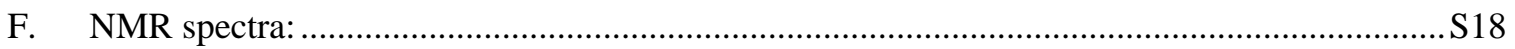

\section{A. General information:}

All reactions were carried out under an argon atmosphere using Standard Schlenk-Lines or a glovebox (Innovative Technology). All reagents were used as received unless otherwise noted. DME, MeCN and DMF were dried over $\mathrm{CaH}_{2}$. Toluene, 1,4-dioxane and THF were dried over sodium. Analytical thin-layer chromatography was performed with $0.25 \mathrm{~mm}$ coated commercial silica gel plates (TLC Silica Gel $60 \mathrm{~F}_{254}$ ); visualization of the developed chromatogram was performed by fluorescence. Flash Chromatography was performed with silica gel (300-400 mesh). Proton nuclear magnetic resonance ( ${ }^{1} \mathrm{H}$ NMR) data were acquired on a WNMR-I $400(400 \mathrm{MHz})$ or Bruker Ascend $400(400 \mathrm{MHz})$ spectrometer, and chemical shifts are reported in delta $(\delta)$ units, in parts per million $(\mathrm{ppm})$ downfield from tetramethylsilane. Splitting patterns are designated as s, singlet; $d$, doublet; dd, doublet of doublets; $t$, triplet; q, quartet; m, multiplet, coupling constants $J$ are quoted in $\mathrm{Hz}$. Carbon-13 nuclear magnetic resonance $\left({ }^{13} \mathrm{C} \mathrm{NMR}\right)$ data were acquired at $100 \mathrm{MHz}$ on a WNMR-I $400(400 \mathrm{MHz})$ or Bruker Ascend $400(400 \mathrm{MHz})$ spectrometer, and chemical shifts are reported in ppm relative to the center line of a triplet at $77.0 \mathrm{ppm}$ for chloroform- $d$ and a heptet at $40.0 \mathrm{ppm}$ for dimethyl sulfoxide- $d_{6}$. Infrared spectra were recorded on a TENSOR 27 FT-IR spectrophotometer and reported in wave numbers $\left(\mathrm{cm}^{-1}\right)$. High resolution mass spectra were acquired on a Bruker Daltonics MicroTof-Q II mass spectrometer. HPLC analyses were performed on an Agilent Technologies 1260 Series using Daicel Chiralpak columns (IA, IB, IC,) in $n$-hexane/i-PrOH. Optical rotations were measured on a Rudolph Research Analytical Autopol II automatic polarimeter. Substrate $\mathbf{1 p}^{1}$ and conjugated dienes and triene $\mathbf{2} \mathbf{a}-\mathbf{g}^{2}, \mathbf{2} \mathbf{i}^{3}$ and $\mathbf{2} \mathbf{j}^{4}$ were prepared according to literature methods. 


\section{B. Preparation of substrates:}

General procedure for the preparation of phenol substrates 1a-e, g-l:

The following phenol substrates 1a-e, $\mathbf{g}$-l were prepared by Suzuki-Miyaura coupling reaction between (4-methoxy-3,5-dimethylphenyl)boronic acid and corresponding ortho-bromoaniline derivatives, followed by Sandmeyer reaction ${ }^{5}$ and demethylation with $\mathrm{BBr}_{3}$.

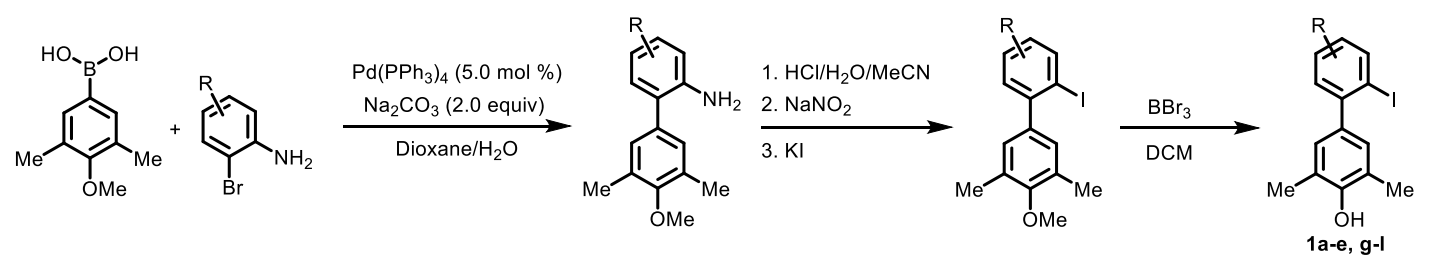

A $25 \mathrm{~mL}$ round bottom flask with a stir bar was fitted with a rubber septum and flame dried under high vacuum. The flask was purged with argon and charged with $\mathrm{Pd}\left(\mathrm{PPh}_{3}\right)_{4}(0.14 \mathrm{~g}, 0.12 \mathrm{mmol}), \mathrm{Na}_{2} \mathrm{CO}_{3}(0.53$ g, $5.0 \mathrm{mmol})$, (4-methoxy-3,5-dimethylphenyl)boronic acid $(0.54 \mathrm{~g}, 3.0 \mathrm{mmol})$, ortho-bromoanilin derivatives $(2.5 \mathrm{mmol}), 10.0 \mathrm{~mL}$ deoxygenated dioxane and $2.0 \mathrm{~mL}$ deoxygenated water. The mixture was stirred at $80{ }^{\circ} \mathrm{C}$ until the reaction was judged to be completed by TLC analysis. Water was added and extracted with ethyl acetate. The organic phase was dried over anhydrous $\mathrm{MgSO}_{4}$ and concentrated under reduced pressure. The residue was then chromatographed on silica gel to afford the desired compound.

To a solution of the above product dissolved in $\mathrm{MeCN}(8.0 \mathrm{~mL})$ was added aq. $\mathrm{HCl}(1.5 \mathrm{~mL}$ conc. $\mathrm{HCl}$ in $5.0 \mathrm{~mL}$ water), then the mixture was cooled to $0{ }^{\circ} \mathrm{C}$, and it was added a solution of $\mathrm{NaNO}_{2}(0.24 \mathrm{~g}$ in 5.0 $\mathrm{mL}$ water). After addition, the reaction was kept at the temperature lower than $5{ }^{\circ} \mathrm{C}$ for $1.0 \mathrm{~h}$ and it was added a solution of $\mathrm{KI}(0.73 \mathrm{~g}$ in $5.0 \mathrm{~mL}$ water). The reaction was kept at room temperature overnight. Water was added and extracted with ethyl acetate. The organic phase was dried over anhydrous $\mathrm{MgSO}_{4}$ and concentrated under reduced pressure. The residue was then chromatographed on silica gel to afford the desired compound.

Under an argon atmosphere, a solution of $\mathrm{BBr}_{3}(2.0$ equiv) in $\mathrm{DCM}(1.0 \mathrm{~mL})$ was slowly added to a solution of the above product in DCM $(20.0 \mathrm{~mL})$ at $0{ }^{\circ} \mathrm{C}$ and the mixture was stirred at room temperature for $3 \mathrm{~h}$. After cooling to $0{ }^{\circ} \mathrm{C}$, the reaction was quenched with cold water and extracted with DCM. The organic phase was dried over anhydrous $\mathrm{MgSO}_{4}$ and concentrated under reduced pressure. The residue was then chromatographed on silica gel to afford the desired product.

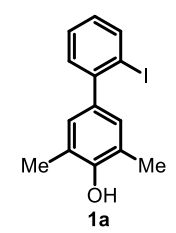

\section{2'-Iodo-3,5-dimethyl-[1,1'-biphenyl]-4-ol (1a)}

Starting material $=2$-bromoaniline. White solid $(0.66 \mathrm{~g}, 82 \%$ yield $) .{ }^{1} \mathrm{H}$ NMR $\left(400 \mathrm{MHz}, \mathrm{CDCl}_{3}\right): \delta$ $7.92(\mathrm{~d}, J=7.8 \mathrm{~Hz}, 1 \mathrm{H}), 7.33(\mathrm{t}, J=7.2 \mathrm{~Hz}, 1 \mathrm{H}), 7.29-7.23(\mathrm{~m}, 1 \mathrm{H}), 7.02-6.92(\mathrm{~m}, 3 \mathrm{H}), 4.68(\mathrm{~s}, 1 \mathrm{H}), 2.29$ (s, 6H). ${ }^{13} \mathrm{C}$ NMR $\left(100 \mathrm{MHz}, \mathrm{CDCl}_{3}\right): \delta 151.8,146.5,139.4,136.4,130.2,129.6,128.4,128.1,122.5,99.2$, 16.0. IR (KBr): 3447, 3020, 2920, 1606, 1491, 1461, 1385, 878, 754, $722 \mathrm{~cm}^{-1}$. HRMS (ESI) m/z calculated for $\mathrm{C}_{14} \mathrm{H}_{13} \mathrm{IONa}[\mathrm{M}+\mathrm{Na}]^{+} 346.9909$, found 346.9901 .

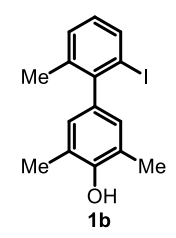




\section{2'-Iodo-3,5,6'-trimethyl-[1,1'-biphenyl]-4-ol (1b)}

Starting material $=2$-bromo-3-methylaniline. Yellow solid $(0.54 \mathrm{~g}, 64 \%$ yield $) .{ }^{1} \mathrm{H}$ NMR $(400 \mathrm{MHz}$, $\left.\mathrm{CDCl}_{3}\right): \delta 7.61(\mathrm{~d}, J=7.9 \mathrm{~Hz}, 1 \mathrm{H}), 7.04(\mathrm{~d}, J=7.5 \mathrm{~Hz}, 1 \mathrm{H}), 6.74(\mathrm{t}, J=7.7 \mathrm{~Hz}, 1 \mathrm{H}), 6.60(\mathrm{~s}, 2 \mathrm{H}), 4.67(\mathrm{~s}$, $1 \mathrm{H}), 2.14(\mathrm{~s}, 6 \mathrm{H}), 1.97(\mathrm{~s}, 3 \mathrm{H}) .{ }^{13} \mathrm{C}$ NMR $\left(100 \mathrm{MHz}, \mathrm{CDCl}_{3}\right): \delta 151.4,146.2,138.0,136.5,136.4,129.7$, 129.1, 128.7, 122.8, 102.0, 22.5, 16.1. IR (KBr): 3463, 3023, 2921, 2857, 1604, 1553, 1490, 1442, 877, 768 , $742 \mathrm{~cm}^{-1}$. HRMS (ESI) $\mathrm{m} / \mathrm{z}$ calculated for $\mathrm{C}_{15} \mathrm{H}_{15} \mathrm{IONa}[\mathrm{M}+\mathrm{Na}]^{+} 361.0065$, found 361.0050 .

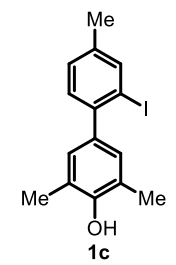

2'-Iodo-3,5,5'-trimethyl-[1,1'-biphenyl]-4-ol (1c)

Starting material $=2$-bromo-5-methylaniline. White solid $(0.63 \mathrm{~g}, 75 \%$ yield $) .{ }^{1} \mathrm{H}$ NMR $(400 \mathrm{MHz}$, $\left.\mathrm{CDCl}_{3}\right): \delta 7.77(\mathrm{~s}, 1 \mathrm{H}), 7.15(\mathrm{~s}, 2 \mathrm{H}), 6.95(\mathrm{~s}, 2 \mathrm{H}), 4.66(\mathrm{~s}, 1 \mathrm{H}), 2.33(\mathrm{~s}, 3 \mathrm{H}), 2.29(\mathrm{~s}, 6 \mathrm{H}) \cdot{ }^{13} \mathrm{C}$ NMR $(100$ $\left.\mathrm{MHz}, \mathrm{CDCl}_{3}\right): \delta 151.7,143.6,139.9,138.4,136.2,129.9,129.7,128.9,122.4,99.1,20.5,16.0 . \mathrm{IR}(\mathrm{KBr}):$ 3450, 3025, 2920, 2859, 1598, 1499, 1474, 1384, 878, 821, $764 \mathrm{~cm}^{-1}$. HRMS (ESI) m/z calculated for $\mathrm{C}_{15} \mathrm{H}_{15} \mathrm{IONa}[\mathrm{M}+\mathrm{Na}]^{+} 361.0065$, found 361.0051 .

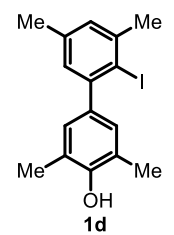

\section{2'-Iodo-3,3',5,5'-tetramethyl-[1,1'-biphenyl]-4-ol (1d)}

Starting material $=2$-bromo-4,6-dimethylaniline. White solid $(0.64 \mathrm{~g}, 73 \%$ yield $) .{ }^{1} \mathrm{H}$ NMR $(400 \mathrm{MHz}$, $\left.\mathrm{CDCl}_{3}\right): \delta 7.02(\mathrm{~d}, J=1.5 \mathrm{~Hz}, 1 \mathrm{H}), 6.92(\mathrm{~s}, 2 \mathrm{H}), 6.89(\mathrm{~s}, 1 \mathrm{H}), 4.65$ (s, 1H), 2.49 (s, 3H), 2.29 (s, 6H), 2.27 (s, 3H). ${ }^{13} \mathrm{C}$ NMR $\left(100 \mathrm{MHz}, \mathrm{CDCl}_{3}\right): \delta 151.6,147.5,142.0,137.8,137.3,129.6,129.2,128.4,122.4$, 102.6, 30.0, 20.7, 16.1. IR (KBr): 3422, 3022, 2923, 2861, 1605, 1528, 1453, 1385, 895, 863, $764 \mathrm{~cm}^{-1}$. HRMS (ESI) $\mathrm{m} / \mathrm{z}$ calculated for $\mathrm{C}_{16} \mathrm{H}_{17} \mathrm{IONa}[\mathrm{M}+\mathrm{Na}]^{+} 375.0222$, found 375.0211 .

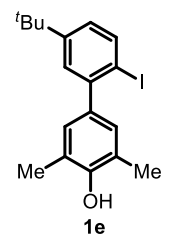

\section{5'-(Tert-butyl)-2'-iodo-3,5-dimethyl-[1,1'-biphenyl]-4-ol (1e)}

Starting material $=2$-bromo-4-(tert-butyl)aniline. Yellow solid $(0.63 \mathrm{~g}, 66 \%$ yield $) .{ }^{1} \mathrm{H}$ NMR $(400$ $\left.\mathrm{MHz}, \mathrm{CDCl}_{3}\right): \delta 7.81(\mathrm{~d}, J=8.3 \mathrm{~Hz}, 1 \mathrm{H}), 7.29(\mathrm{~d}, J=2.5 \mathrm{~Hz}, 1 \mathrm{H}), 7.02-6.98(\mathrm{~m}, 1 \mathrm{H}), 6.97(\mathrm{~s}, 2 \mathrm{H}), 4.77(\mathrm{~s}$, $1 \mathrm{H}), 2.28(\mathrm{~s}, 6 \mathrm{H}), 1.29$ (s, 9H). ${ }^{13} \mathrm{C}$ NMR $\left(100 \mathrm{MHz}, \mathrm{CDCl}_{3}\right): \delta 151.7,151.4,145.9,139.0,136.8,129.6$, 127.6, 125.9, 122.5, 95.4, 34.6, 31.3, 16.1. IR (KBr): 3444, 3026, 2963, 2867, 1606, 1523, 1489, 1460, $1383,1362,875,818,772,734 \mathrm{~cm}^{-1}$. HRMS (ESI) $\mathrm{m} / \mathrm{z}$ calculated for $\mathrm{C}_{18} \mathrm{H}_{21} \mathrm{IONa}[\mathrm{M}+\mathrm{Na}]^{+} 403.0535$, found 403.0518 . 


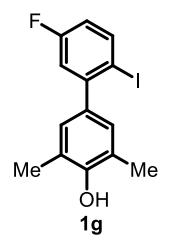

\section{5'-Fluoro-2'-iodo-3,5-dimethyl-[1,1'-biphenyl]-4-ol (1g)}

Starting material $=2$-bromo-4-fluoroaniline. Yellow solid $(0.60 \mathrm{~g}, 70 \%$ yield $) .{ }^{1} \mathrm{H}$ NMR $(400 \mathrm{MHz}$, $\left.\mathrm{CDCl}_{3}\right): \delta$ 7.87-7.81 (m, 1H), 7.04-6.99 (m, 1H), $6.95(\mathrm{~s}, 2 \mathrm{H}), 6.79-6.71(\mathrm{~m}, 1 \mathrm{H}), 4.72(\mathrm{~s}, 1 \mathrm{H}), 2.29(\mathrm{~s}, 6 \mathrm{H})$. ${ }^{13} \mathrm{C}$ NMR $\left(100 \mathrm{MHz}, \mathrm{CDCl}_{3}\right): \delta 162.8(\mathrm{~d}, J=246.6 \mathrm{~Hz}), 152.1,148.4(\mathrm{~d}, J=7.7 \mathrm{~Hz}), 140.5(\mathrm{~d}, J=7.8 \mathrm{~Hz})$, $135.4(\mathrm{~d}, J=2.3 \mathrm{~Hz}), 129.4,122.7,117.4(\mathrm{~d}, J=21.8 \mathrm{~Hz}), 115.7$ (d, $J=21.5 \mathrm{~Hz}), 92.0,16.0$. IR $(\mathrm{KBr})$ : 3448, 3021, 2921, 1596, 1569, 1491, 1386, 1261, 869, 808, $764 \mathrm{~cm}^{-1}$. HRMS (ESI) m/z calculated for $\mathrm{C}_{14} \mathrm{H}_{12} \mathrm{IFONa}[\mathrm{M}+\mathrm{Na}]^{+} 364.9815$, found 364.9802 .

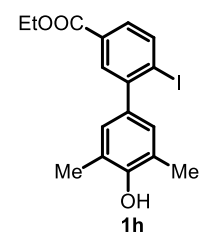

\section{Ethyl 4'-hydroxy-6-iodo-3',5'-dimethyl-[1,1'-biphenyl]-3-carboxylate (1h)}

Starting material = ethyl 4-amino-3-bromobenzoate. White solid $(0.66 \mathrm{~g}, 67 \%$ yield $) .{ }^{1} \mathrm{H}$ NMR (400 $\left.\mathrm{MHz}, \mathrm{CDCl}_{3}\right): \delta 8.01(\mathrm{~d}, J=8.2 \mathrm{~Hz}, 1 \mathrm{H}), 7.91(\mathrm{~d}, J=2.1 \mathrm{~Hz}, 1 \mathrm{H}), 7.65-7.60(\mathrm{~m}, 1 \mathrm{H}), 6.97(\mathrm{~s}, 2 \mathrm{H}), 4.82(\mathrm{~s}$, $1 \mathrm{H}), 4.37(\mathrm{q}, J=7.1 \mathrm{~Hz}, 2 \mathrm{H}), 2.31(\mathrm{~s}, 6 \mathrm{H}), 1.38(\mathrm{t}, J=7.1 \mathrm{~Hz}, 3 \mathrm{H}) .{ }^{13} \mathrm{C}$ NMR $\left(100 \mathrm{MHz}, \mathrm{CDCl}_{3}\right): \delta 166.4$, 152.1, 146.9, 139.7, 135.5, 130.7, 130.4, 129.5, 128.9, 122.7, 105.5, 61.3, 16.0, 14.3. IR (KBr): 3454, 3026, 2984, 1701, 1587, 1559, 1490, 1458, 1386, 1315, 877, 849, $762 \mathrm{~cm}^{-1}$. HRMS (ESI) m/z calculated for $\mathrm{C}_{17} \mathrm{H}_{17} \mathrm{IO}_{3} \mathrm{Na}[\mathrm{M}+\mathrm{Na}]^{+}$419.0120, found 419.0106.

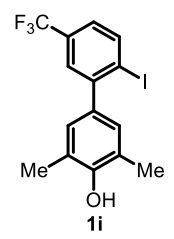

2'-Iodo-3,5-dimethyl-5'-(trifluoromethyl)-[1,1'-biphenyl]-4-ol (1i)

Starting material $=2$-bromo-4-(trifluoromethyl)aniline. Yellow solid $\left(0.70 \mathrm{~g}, 72 \%\right.$ yield). ${ }^{1} \mathrm{H}$ NMR $\left(400 \mathrm{MHz}, \mathrm{CDCl}_{3}\right): \delta 8.04(\mathrm{~d}, J=8.2 \mathrm{~Hz}, 1 \mathrm{H}), 7.51(\mathrm{~d}, J=2.1 \mathrm{~Hz}, 1 \mathrm{H}), 7.26-7.18(\mathrm{~m}, 1 \mathrm{H}), 6.97(\mathrm{~s}, 2 \mathrm{H})$, $4.77(\mathrm{~s}, 1 \mathrm{H}), 2.30(\mathrm{~s}, 6 \mathrm{H}) .{ }^{13} \mathrm{C} \mathrm{NMR}\left(100 \mathrm{MHz}, \mathrm{CDCl}_{3}\right): \delta 152.3,147.4,140.1,135.1,130.6$ (q, $J=32.5$ $\mathrm{Hz}), 129.4,126.5(\mathrm{q}, J=3.7 \mathrm{~Hz}), 124.7(\mathrm{q}, J=3.6 \mathrm{~Hz}), 124.0(\mathrm{q}, J=270.8 \mathrm{~Hz}), 122.8,103.6,16.0 . \mathrm{IR}$ (KBr): 3373, 3027, 2912, 2858, 1599, 1570, 1489, 1463, 1167, 870, 823, $798 \mathrm{~cm}^{-1}$. HRMS (ESI) m/z calculated for $\mathrm{C}_{15} \mathrm{H}_{12} \mathrm{IF}_{3} \mathrm{ONa}[\mathrm{M}+\mathrm{Na}]^{+} 414.9783$, found 414.9772 .

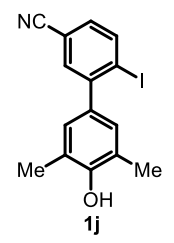

4'-Hydroxy-6-iodo-3',5'-dimethyl-[1,1'-biphenyl]-3-carbonitrile (1j)

Starting material $=4$-amino-3-bromobenzonitrile. Yellow solid $(0.51 \mathrm{~g}, 59 \%$ yield $) .{ }^{1} \mathrm{H}$ NMR (400 $\left.\mathrm{MHz}, \mathrm{CDCl}_{3}\right): \delta 7.97(\mathrm{~d}, J=8.2 \mathrm{~Hz}, 1 \mathrm{H}), 7.44(\mathrm{~d}, J=2.0 \mathrm{~Hz}, 1 \mathrm{H}), 7.16-7.12(\mathrm{~m}, 1 \mathrm{H}), 6.85(\mathrm{~s}, 2 \mathrm{H}), 4.90(\mathrm{~s}$, $1 \mathrm{H}), 2.22$ (s, 6H). ${ }^{13} \mathrm{C}$ NMR (100 MHz, $\left.\mathrm{CDCl}_{3}\right): \delta 152.6,148.0,140.5,134.2,132.8,130.9,129.3,123.1$, 
118.3, 112.1, 105.7, 16.1. IR (KBr): 3443, 3027, 2919, 2232, 1636, 1490, 1458, 1385, 1317, 873, 820, 764 $\mathrm{cm}^{-1}$. HRMS (ESI) $\mathrm{m} / \mathrm{z}$ calculated for $\mathrm{C}_{15} \mathrm{H}_{12} \mathrm{INONa}[\mathrm{M}+\mathrm{Na}]^{+} 371.9861$, found 371.9845.

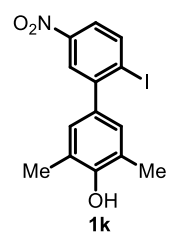

2'-Iodo-3,5-dimethyl-5'-nitro-[1,1'-biphenyl]-4-ol (1k)

Starting material $=2$-bromo-4-nitroaniline. Yellow solid $(0.52 \mathrm{~g}, 56 \%$ yield $) .{ }^{1} \mathrm{H}$ NMR $(400 \mathrm{MHz}$, $\left.\mathrm{CDCl}_{3}\right): \delta$ 8.16-8.11 (m, 2H), 7.86-7.82 (m, 1H), $7.01(\mathrm{~s}, 2 \mathrm{H}), 4.85(\mathrm{~s}, 1 \mathrm{H}), 2.34(\mathrm{~s}, 6 \mathrm{H}) \cdot{ }^{13} \mathrm{C}$ NMR $(100$ $\left.\mathrm{MHz}, \mathrm{CDCl}_{3}\right): \delta 152.5,148.3,148.1,140.5,134.3,129.3,124.2,123.0,122.4,107.5,15.9$. IR (KBr): 3443 , 3030, 2918, 1636, 1516, 1488, 1456, 1384, 1275, 871, 824, $764 \mathrm{~cm}^{-1}$. HRMS (ESI) m/z calculated for $\mathrm{C}_{14} \mathrm{H}_{12} \mathrm{INO}_{3} \mathrm{Na}[\mathrm{M}+\mathrm{Na}]^{+}$391.9760, found 391.9742.

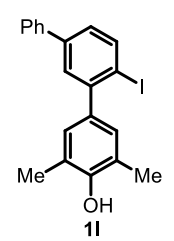

6'-Iodo-3,5-dimethyl-[1,1':3',1'-terphenyl]-4-ol (11)

Starting material $=3$-bromo-[1,1'-biphenyl]-4-amine. White solid $\left(0.78 \mathrm{~g}, 78 \%\right.$ yield). ${ }^{1} \mathrm{H}$ NMR (400 $\left.\mathrm{MHz}, \mathrm{CDCl}_{3}\right): \delta 7.91(\mathrm{~d}, J=8.2 \mathrm{~Hz}, 1 \mathrm{H}), 7.55-7.50(\mathrm{~m}, 2 \mathrm{H}), 7.49(\mathrm{~d}, J=2.3 \mathrm{~Hz}, 1 \mathrm{H}), 7.38-7.32(\mathrm{~m}, 2 \mathrm{H})$, 7.31-7.27 (m, 1H), 7.17-7.12 (m, 1H), $6.99(\mathrm{~s}, 2 \mathrm{H}), 4.75(\mathrm{~s}, 1 \mathrm{H}), 2.25(\mathrm{~s}, 6 \mathrm{H}) .{ }^{13} \mathrm{C} \mathrm{NMR}\left(100 \mathrm{MHz}, \mathrm{CDCl}_{3}\right)$ : $\delta$ 152.1, 147.1, 141.3, 140.1, 140.0, 136.5, 129.8, 129.1, 129.0, 127.9, 127.2, 127.1, 122.8, 98.1, 16.2. IR (KBr): 3446, 3030, 2922, 1604, 1491, 1462, 1379, 878, 825, 790, 762, $699 \mathrm{~cm}^{-1}$. HRMS (ESI) m/z calculated for $\mathrm{C}_{20} \mathrm{H}_{17} \mathrm{IONa}[\mathrm{M}+\mathrm{Na}]^{+} 423.0222$, found 423.0204 .

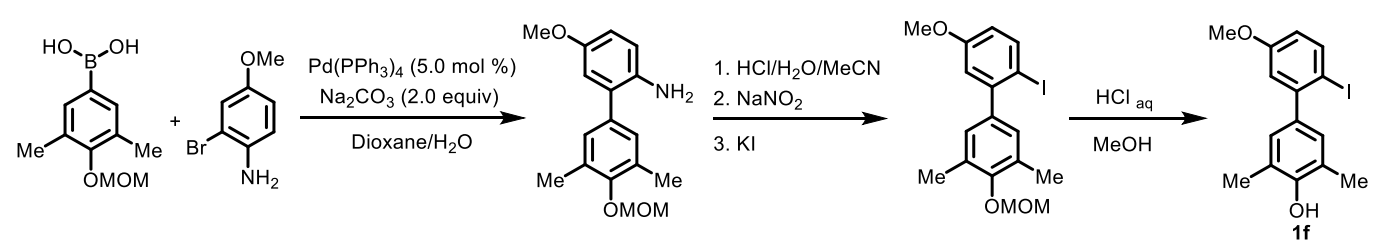

\section{2'-Iodo-5'-methoxy-3,5-dimethyl-[1,1'-biphenyl]-4-ol (1f)}

A $25 \mathrm{~mL}$ round bottom flask with a stir bar was fitted with a rubber septum and flame dried under high vacuum. The flask was purged with argon and charged with $\mathrm{Pd}\left(\mathrm{PPh}_{3}\right)_{4}(0.14 \mathrm{~g}, 0.12 \mathrm{mmol}), \mathrm{Na}_{2} \mathrm{CO}_{3}(0.53 \mathrm{~g}$, $5.0 \mathrm{mmol}$ ), (4-(methoxymethoxy)-3,5-dimethylphenyl)boronic acid $(0.63 \mathrm{~g}, 3.0 \mathrm{mmol})$, 2-bromo-4methoxyaniline $(0.50 \mathrm{~g}, 2.5 \mathrm{mmol}), 10.0 \mathrm{~mL}$ deoxygenated dioxane and $2.0 \mathrm{~mL}$ deoxygenated water. The mixture was stirred at $80{ }^{\circ} \mathrm{C}$ until the reaction was judged to be completed by TLC analysis. Water was added and extracted with ethyl acetate. The organic phase was dried over anhydrous $\mathrm{MgSO}_{4}$ and concentrated under reduced pressure. The residue was then chromatographed on silica gel to afford the desired compound.

To a solution of the above product dissolved in $\mathrm{MeCN}(8.0 \mathrm{~mL})$ was added aq. $\mathrm{HCl}(1.5 \mathrm{~mL}$ conc. $\mathrm{HCl}$ in $5.0 \mathrm{~mL}$ water), then the mixture was cooled to $0{ }^{\circ} \mathrm{C}$, and it was added a solution of $\mathrm{NaNO}_{2}(0.24 \mathrm{~g}$ in 5.0 $\mathrm{mL}$ water). After addition, the reaction was kept at the temperature lower than $5{ }^{\circ} \mathrm{C}$ for $1.0 \mathrm{~h}$ and it was added a solution of $\mathrm{KI}(0.73 \mathrm{~g}$ in $5.0 \mathrm{~mL}$ water). The reaction was kept at room temperature overnight. Water was added and extracted with ethyl acetate. The organic phase was dried over anhydrous $\mathrm{MgSO}_{4}$ and 
concentrated under reduced pressure. The residue was then chromatographed on silica gel to afford the desired compound.

The above product was dissolved in $30.0 \mathrm{~mL} \mathrm{MeOH}$ in a $100 \mathrm{~mL}$ round bottom flask, warmed to $70{ }^{\circ} \mathrm{C}$ and conc. $\mathrm{HCl}$ aq. (35\%, 3 drops) was added. After stirring for $3 \mathrm{~h}$, the flask was removed from the oil bath and cooled to room temperature, $40.0 \mathrm{~mL}$ of distilled water was added and extracted with ethyl acetate. The organic phase was dried over anhydrous $\mathrm{MgSO}_{4}$ and concentrated under reduced pressure to give a crude product of $\mathbf{1 f}$, which was purified by column chromatography to afford $\mathbf{1 f}$ as a brown solid $(0.51 \mathrm{~g}, 58 \%$ yield). ${ }^{1} \mathrm{H}$ NMR (400 MHz, $\left.\mathrm{CDCl}_{3}\right): \delta 7.77(\mathrm{~d}, J=8.7 \mathrm{~Hz}, 1 \mathrm{H}), 6.97(\mathrm{~s}, 2 \mathrm{H}), 6.85(\mathrm{~d}, J=2.9 \mathrm{~Hz}, 1 \mathrm{H}), 6.64-$ $6.58(\mathrm{~m}, 1 \mathrm{H}), 4.70(\mathrm{~s}, 1 \mathrm{H}), 3.79(\mathrm{~s}, 3 \mathrm{H}), 2.30(\mathrm{~s}, 6 \mathrm{H}) .{ }^{13} \mathrm{C} \mathrm{NMR}\left(100 \mathrm{MHz}, \mathrm{CDCl}_{3}\right): \delta 159.7,151.8,147.4$, 139.9, 136.2, 129.4, 122.5, 115.9, 114.9, 87.7, 55.4, 16.0. IR (KBr): 3445, 3025, 2920, 2908, 1587, 1563, 1492, 1464, 1387, 1263, 877, 814, $766 \mathrm{~cm}^{-1}$. HRMS (ESI) $\mathrm{m} / \mathrm{z}$ calculated for $\mathrm{C}_{15} \mathrm{H}_{15} \mathrm{IO}_{2} \mathrm{Na}[\mathrm{M}+\mathrm{Na}]^{+}$ 377.0014, found 377.0004 .

\section{General procedure for the preparation of phenol substrates 1m-n:}

The following phenol substrates were prepared by Suzuki-Miyaura reaction between substituted (4methoxyphenyl)boronic acid and 2-bromoaniline, followed by Sandmeyer reaction and demethylation with $\mathrm{BBr}_{3}$.

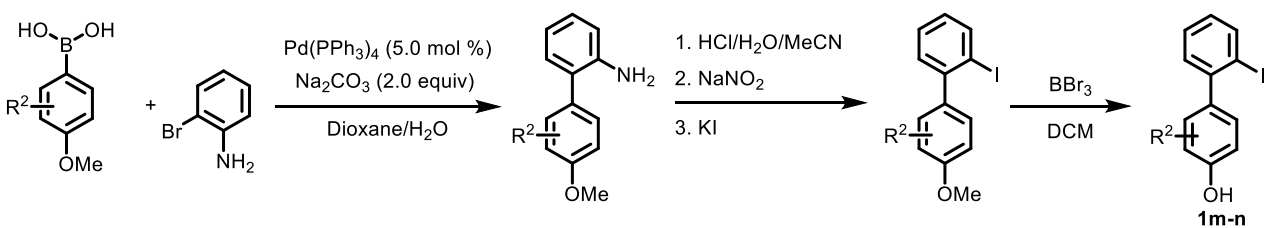

A $25 \mathrm{~mL}$ round bottom flask with a stir bar was fitted with a rubber septum and flame dried under high vacuum. The flask was purged with argon and charged with $\mathrm{Pd}\left(\mathrm{PPh}_{3}\right)_{4}(0.14 \mathrm{~g}, 0.12 \mathrm{mmol}), \mathrm{Na}_{2} \mathrm{CO}_{3}(0.53$ $\mathrm{g}, 5.0 \mathrm{mmol})$, substituted (4-methoxyphenyl)boronic acid $(3.0 \mathrm{mmol})$, 2-bromoaniline $(0.43 \mathrm{~g}, 2.5 \mathrm{mmol})$, $10.0 \mathrm{~mL}$ deoxygenated dioxane and $2.0 \mathrm{~mL}$ deoxygenated water. The mixture was stirred at $80{ }^{\circ} \mathrm{C}$ until the reaction was judged to be completed by TLC analysis. Water was added and extracted with ethyl acetate. The organic phase was dried over anhydrous $\mathrm{MgSO}_{4}$ and concentrated under reduced pressure. The residue was then chromatographed on silica gel to afford the desired compound.

To a solution of the above product dissolved in $\mathrm{MeCN}(8.0 \mathrm{~mL})$ was added aq. $\mathrm{HCl}(1.5 \mathrm{~mL}$ conc. $\mathrm{HCl}$ in $5.0 \mathrm{~mL}$ water), then the mixture was cooled to $0{ }^{\circ} \mathrm{C}$, and it was added a solution of $\mathrm{NaNO}_{2}(0.24 \mathrm{~g}$ in 5.0 $\mathrm{mL}$ water). After addition, the reaction was kept at the temperature lower than $5{ }^{\circ} \mathrm{C}$ for $1.0 \mathrm{~h}$ and it was added a solution of $\mathrm{KI}(0.73 \mathrm{~g}$ in $5.0 \mathrm{~mL}$ water). The reaction was kept at room temperature overnight. Water was added and extracted with ethyl acetate. The organic phase was dried over anhydrous $\mathrm{MgSO}_{4}$ and concentrated under reduced pressure. The residue was then chromatographed on silica gel to afford the desired compound.

Under an argon atmosphere, a solution of $\mathrm{BBr}_{3}(2.0$ equiv) in DCM $(1.0 \mathrm{~mL})$ was slowly added to a solution of the above product in DCM $(20.0 \mathrm{~mL})$ at $0{ }^{\circ} \mathrm{C}$ and the mixture was stirred at room temperature for $3 \mathrm{~h}$. After cooling to $0{ }^{\circ} \mathrm{C}$, the reaction was quenched with cold water and extracted with DCM. The organic phase was dried over anhydrous $\mathrm{MgSO}_{4}$ and concentrated under reduced pressure. The residue was then chromatographed on silica gel to afford the desired product $\mathbf{1 m}-\mathbf{n}$.

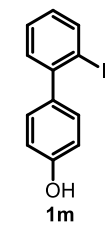

\section{2'-Iodo-[1,1'-biphenyl]-4-ol (1m)}


Starting material $=\left(4\right.$-methoxyphenyl)boronic acid. White solid $(0.56 \mathrm{~g}, 76 \%$ yield $) .{ }^{1} \mathrm{H}$ NMR $(400$ $\left.\mathrm{MHz}, \mathrm{CDCl}_{3}\right): \delta$ 7.96-7.92 (m, 1H), 7.39-7.34 (m, 1H), 7.30-7.27 (m, 1H), 7.25-7.24 (m, 1H), 7.23-7.21 (m, $1 \mathrm{H}), 7.04-6.98(\mathrm{~m}, 1 \mathrm{H}), 6.93-6.85(\mathrm{~m}, 2 \mathrm{H}), 4.92(\mathrm{~s}, 1 \mathrm{H}) .{ }^{13} \mathrm{C}$ NMR $\left(100 \mathrm{MHz}, \mathrm{CDCl}_{3}\right): \delta 155.0,146.2$, 139.5, 137.1, 130.7, 130.2, 128.6, 128.2, 114.9, 99.2. IR (KBr): 3160, 1896, 1609, 1591, 1515, 1459, 835, $820,760 \mathrm{~cm}^{-1}$. HRMS (ESI) $\mathrm{m} / \mathrm{z}$ calculated for $\mathrm{C}_{12} \mathrm{H}_{9} \mathrm{IONa}[\mathrm{M}+\mathrm{Na}]^{+} 318.9596$, found 318.9583 .

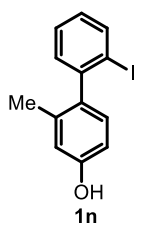

2'-Iodo-2-methyl-[1,1'-biphenyl]-4-ol (1n)

Starting material $=(4$-methoxy-2-methylphenyl $)$ boronic acid. White solid $(0.56 \mathrm{~g}, 72 \%$ yield $) .{ }^{1} \mathrm{H}$ NMR (400 MHz, DMSO- $\left.d_{6}\right): \delta 9.40(\mathrm{~s}, 1 \mathrm{H}), 7.93(\mathrm{~d}, J=7.8 \mathrm{~Hz}, 1 \mathrm{H}), 7.43(\mathrm{t}, J=7.2 \mathrm{~Hz}, 1 \mathrm{H}), 7.20(\mathrm{~d}, J=$ $7.4 \mathrm{~Hz}, 1 \mathrm{H}), 7.08(\mathrm{t}, J=7.1 \mathrm{~Hz}, 1 \mathrm{H}), 6.80(\mathrm{~d}, J=8.1 \mathrm{~Hz}, 1 \mathrm{H}), 6.67(\mathrm{~s}, 1 \mathrm{H}), 6.63(\mathrm{~d}, J=8.2 \mathrm{~Hz}, 1 \mathrm{H}), 1.91(\mathrm{~s}$, $3 \mathrm{H}) .{ }^{13} \mathrm{C}$ NMR $\left(100 \mathrm{MHz}, \mathrm{DMSO}-d_{6}\right): \delta 157.3,146.7,138.9,136.5,135.6,130.7,130.4,129.3,128.7$, 116.8, 113.0, 102.0, 20.3. IR (KBr): 2426, 3054, 2921, 1610, 1585, 1505, 1457, 1384, 896, 813, 763, 750 $\mathrm{cm}^{-1}$. HRMS (ESI) $\mathrm{m} / \mathrm{z}$ calculated for $\mathrm{C}_{13} \mathrm{H}_{11} \mathrm{IONa}[\mathrm{M}+\mathrm{Na}]^{+} 332.9752$, found 332.9737 .

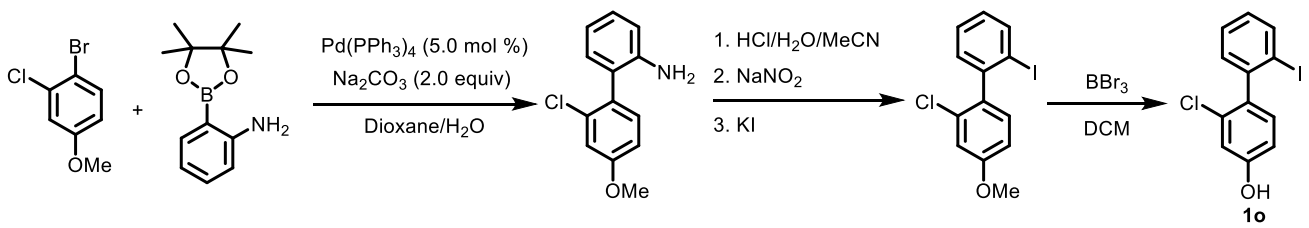

\section{2-Chloro-2'-iodo-[1,1'-biphenyl]-4-ol (10)}

A $25 \mathrm{~mL}$ round bottom flask with a stir bar was fitted with a rubber septum and flame dried under high vacuum. The flask was purged with argon and charged with $\mathrm{Pd}\left(\mathrm{PPh}_{3}\right)_{4}(0.14 \mathrm{~g}, 0.12 \mathrm{mmol}), \mathrm{Na}_{2} \mathrm{CO}_{3}(0.53 \mathrm{~g}$, $5.0 \mathrm{mmol}), 2$-(4,4,5,5-tetramethyl-1,3,2-dioxaborolan-2-yl)aniline (0.66 g, $3.0 \mathrm{mmol}), 1$-bromo-2-chloro-4methoxybenzene $(0.55 \mathrm{~g}, 2.5 \mathrm{mmol}), 10.0 \mathrm{~mL}$ deoxygenated dioxane and $2.0 \mathrm{~mL}$ deoxygenated water. The mixture was stirred at $80{ }^{\circ} \mathrm{C}$ until the reaction was judged to be completed by TLC analysis. Water was added and extracted with ethyl acetate. The organic phase was dried over anhydrous $\mathrm{MgSO}_{4}$ and concentrated under reduced pressure. The residue was then chromatographed on silica gel to afford the desired compound.

To a solution of the above product dissolved in $\mathrm{MeCN}(8.0 \mathrm{~mL})$ was added aq. $\mathrm{HCl}(1.5 \mathrm{~mL}$ conc. $\mathrm{HCl}$ in $5.0 \mathrm{~mL}$ water), then the mixture was cooled to $0{ }^{\circ} \mathrm{C}$, and it was added a solution of $\mathrm{NaNO}_{2}(0.24 \mathrm{~g}$ in 5.0 $\mathrm{mL}$ water). After addition, the reaction was kept at the temperature lower than $5{ }^{\circ} \mathrm{C}$ for $1.0 \mathrm{~h}$ and it was added a solution of $\mathrm{KI}(0.73 \mathrm{~g}$ in $5.0 \mathrm{~mL}$ water). The reaction was kept at room temperature overnight. Water was added and extracted with ethyl acetate. The organic phase was dried over anhydrous $\mathrm{MgSO}_{4}$ and concentrated under reduced pressure. The residue was then chromatographed on silica gel to afford the desired compound.

Under an argon atmosphere, a solution of $\mathrm{BBr}_{3}(2.0$ equiv) in DCM $(1.0 \mathrm{~mL})$ was slowly added to a solution of the above product in DCM $(20.0 \mathrm{~mL})$ at $0{ }^{\circ} \mathrm{C}$ and the mixture was stirred at room temperature for $3 \mathrm{~h}$. After cooling to $0{ }^{\circ} \mathrm{C}$, the reaction was quenched with cold water and extracted with DCM. The organic phase was dried over anhydrous $\mathrm{MgSO}_{4}$ and concentrated under reduced pressure. The residue was then chromatographed on silica gel to afford the desired product $1 \mathrm{o}$ as a white solid $\left(0.46 \mathrm{~g}, 56 \%\right.$ yield). ${ }^{1} \mathrm{H}$ NMR $\left(400 \mathrm{MHz}, \mathrm{DMSO}-d_{6}\right): \delta 10.03(\mathrm{~s}, 1 \mathrm{H}), 7.95-7.91(\mathrm{~m}, 1 \mathrm{H}), 7.46-7.41(\mathrm{~m}, 1 \mathrm{H}), 7.26-7.21(\mathrm{~m}, 1 \mathrm{H})$, 7.14-7.08 (m, 1H), $7.02(\mathrm{~d}, J=8.4 \mathrm{~Hz}, 1 \mathrm{H}), 6.91(\mathrm{~d}, J=2.4 \mathrm{~Hz}, 1 \mathrm{H}), 6.83-6.78(\mathrm{~m}, 1 \mathrm{H}) .{ }^{13} \mathrm{C}$ NMR $(100$ MHz, DMSO- $\left.d_{6}\right): \delta 163.2,149.3,143.7,138.7,137.5,137.0,135.7,134.7,133.4,120.8,119.5,106.4$. IR 
(KBr): 3405, 3028, 2994, 1770, 1758, 1655, 1460, 1246, 902, 852, $758 \mathrm{~cm}^{-1}$. HRMS (ESI) m/z calculated for $\mathrm{C}_{12} \mathrm{H}_{8} \mathrm{IClONa}[\mathrm{M}+\mathrm{Na}]^{+}$352.9206, found 352.9194 .

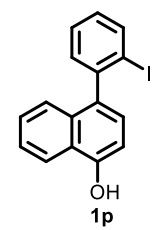

\section{4-(2-Iodophenyl)naphthalen-1-ol (1p)}

White solid $\left(0.55 \mathrm{~g}, 63 \%\right.$ yield). ${ }^{1} \mathrm{H}$ NMR $\left(400 \mathrm{MHz}, \mathrm{CDCl}_{3}\right): \delta 8.26(\mathrm{~d}, J=8.3 \mathrm{~Hz}, 1 \mathrm{H}), 8.00(\mathrm{~d}, J=$ $7.9 \mathrm{~Hz}, 1 \mathrm{H}), 7.52-7.48(\mathrm{~m}, 1 \mathrm{H}), 7.45-7.41(\mathrm{~m}, 2 \mathrm{H}), 7.38-7.32(\mathrm{~m}, 2 \mathrm{H}), 7.15(\mathrm{~d}, J=7.6 \mathrm{~Hz}, 1 \mathrm{H}), 7.13-7.09$ $(\mathrm{m}, 1 \mathrm{H}), 6.87(\mathrm{~d}, J=7.6 \mathrm{~Hz}, 1 \mathrm{H}), 5.35(\mathrm{~s}, 1 \mathrm{H})$. Analytical data are in accordance with the literature values. ${ }^{1}$

\section{Catalytic results:}

General procedure for the $\operatorname{Pd}(0)$-Catalyzed Dearomatization:

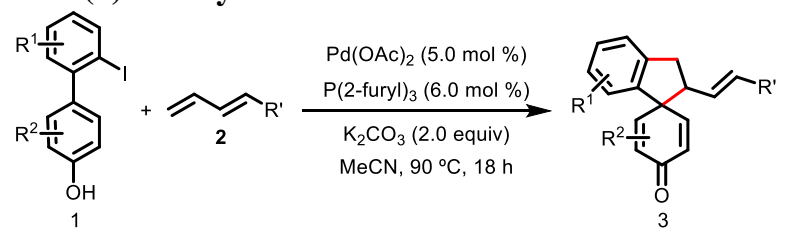

In a glovebox, a $5.0 \mathrm{~mL}$ vial equipped with a stir bar was charged with $\mathrm{Pd}(\mathrm{OAc})_{2}(2.2 \mathrm{mg}, 0.01 \mathrm{mmol})$, tri(2-furyl)phosphine (2.8 $\mathrm{mg}, 0.012 \mathrm{mmol}), \mathrm{K}_{2} \mathrm{CO}_{3}(55.2 \mathrm{mg}, 0.4 \mathrm{mmol})$ and $\mathrm{MeCN}(1.6 \mathrm{~mL})$ was then added. After the catalyst mixture was stirred at room temperature for $10 \mathrm{~min}$, substrate $\mathbf{1}(0.2 \mathrm{mmol})$ and 1,3-diene $2(0.4 \mathrm{mmol})$ were sequentially added. The vial was sealed with a Teflon screw cap and the reaction mixture was heated at $90{ }^{\circ} \mathrm{C}$ for $18 \mathrm{~h}$. After the reaction vessel was cooled to room temperature, the mixture was extracted with ethyl acetate, dried over anhydrous $\mathrm{MgSO}_{4}$ and concentrated under reduced pressure. The residue was then chromatographed on silica gel to afford the desired product 3 .

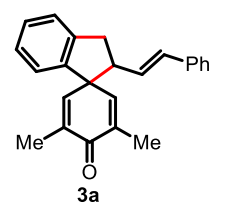

(E)-3,5-Dimethyl-2'-styryl-2',3'-dihydrospiro[cyclohexa[2,5]diene-1,1'-inden]-4-one (3a)

Yellow solid (55.5 mg, 85\% yield). PE/EA $=20: 1, \mathrm{R}_{\mathrm{f}}=0.46 .{ }^{1} \mathrm{H}$ NMR $\left(400 \mathrm{MHz}, \mathrm{CDCl}_{3}\right): \delta 7.35(\mathrm{~d}, J$ $=7.4 \mathrm{~Hz}, 1 \mathrm{H}), 7.31-7.27(\mathrm{~m}, 4 \mathrm{H}), 7.25-7.15(\mathrm{~m}, 3 \mathrm{H}), 6.92(\mathrm{~d}, J=7.5 \mathrm{~Hz}, 1 \mathrm{H}), 6.74(\mathrm{~d}, J=1.3 \mathrm{~Hz}, 1 \mathrm{H})$, $6.66(\mathrm{~d}, J=1.3 \mathrm{~Hz}, 1 \mathrm{H}), 6.45(\mathrm{~d}, J=15.8 \mathrm{~Hz}, 1 \mathrm{H}), 6.11(\mathrm{dd}, J=15.8,7.4 \mathrm{~Hz}, 1 \mathrm{H}), 3.49-3.39(\mathrm{~m}, 1 \mathrm{H})$, 3.35-3.19 (m, 2H), 2.02 (s, 3H), 1.87 (s, 3H). ${ }^{13} \mathrm{C}$ NMR (100 MHz, $\left.\mathrm{CDCl}_{3}\right): \delta$ 187.7, 147.6, 144.4, 143.6, 142.6, 136.9, 136.7, 133.8, 131.8, 128.6, 128.1, 127.7, 127.6, 127.1, 126.3, 125.3, 124.1, 57.4, 54.9, 37.0, 16.4, 16.3. IR (KBr): 3062, 3026, 2924, 2852, 1638, 1602, 1496, 1476, 1449, 1461, 1375, 1264, 966, 761, $751,696 \mathrm{~cm}^{-1}$. HRMS (ESI) m/z calculated for $\mathrm{C}_{24} \mathrm{H}_{22} \mathrm{ONa}[\mathrm{M}+\mathrm{Na}]^{+} 349.1568$, found 349.1553.

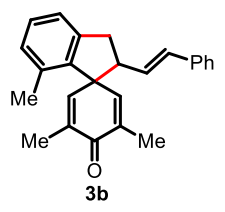

(E)-3,5,7'-Trimethyl-2'-styryl-2',3'-dihydrospiro[cyclohexa[2,5]diene-1,1'-inden]-4-one (3b) 
Yellow solid (57.2 mg, 84\% yield). PE/EA $=20: 1, \mathrm{R}_{\mathrm{f}}=0.44 .{ }^{1} \mathrm{H}$ NMR $\left(400 \mathrm{MHz}, \mathrm{CDCl}_{3}\right): \delta 7.28-7.25$ (m, 4H), 7.24-7.15 (m, 3H), 7.00-6.91 (m, 1H), 6.82-6.77 (m, 1H), 6.63-6.59 (m, 1H), $6.38(\mathrm{~d}, J=15.7 \mathrm{~Hz}$, $1 \mathrm{H}), 6.00(\mathrm{dd}, J=15.8,7.4 \mathrm{~Hz}, 1 \mathrm{H}), 3.41-3.12(\mathrm{~m}, 3 \mathrm{H}), 2.01(\mathrm{~s}, 6 \mathrm{H}), 1.90(\mathrm{~d}, J=1.2 \mathrm{~Hz}, 3 \mathrm{H}) .{ }^{13} \mathrm{C} \mathrm{NMR}$ $\left(100 \mathrm{MHz}, \mathrm{CDCl}_{3}\right): \delta 187.4,148.9,143.2,143.0,140.9,136.9,136.1,135.7,135.2,131.9,129.3,128.6$, 128.2, 127.5, 127.3, 126.3, 122.9, 57.8, 54.8, 37.2, 18.2, 16.3, 16.2. IR (KBr): 3056, 3026, 2923, 2852 , $1633,1495,1460,1447,1398,1264,964,753,748,704 \mathrm{~cm}^{-1}$. HRMS (ESI) $\mathrm{m} / \mathrm{z}$ calculated for $\mathrm{C}_{25} \mathrm{H}_{24} \mathrm{ONa}$ $[\mathrm{M}+\mathrm{Na}]^{+} 363.1725$, found 363.1713 .

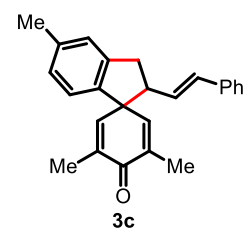

(E)-3,5,5'-Trimethyl-2'-styryl-2',3'-dihydrospiro[cyclohexa[2,5]diene-1,1'-inden]-4-one (3c)

Yellow solid (51.7 mg, 76\% yield). PE/EA = 20:1, $\mathrm{R}_{\mathrm{f}}=0.47 .{ }^{1} \mathrm{H} \mathrm{NMR}\left(400 \mathrm{MHz}, \mathrm{CDCl}_{3}\right): \delta 7.29-7.28$ (m, 3H), 7.27-7.15 (m, 3H), $6.99(\mathrm{~d}, J=7.4 \mathrm{~Hz}, 1 \mathrm{H}), 6.80(\mathrm{~d}, J=7.7 \mathrm{~Hz}, 1 \mathrm{H}), 6.73-6.69(\mathrm{~m}, 1 \mathrm{H}), 6.66-6.62$ (m, 1H), 6.48-6.40 (m, 1H), $6.10(\mathrm{dd}, J=15.8,7.5 \mathrm{~Hz}, 1 \mathrm{H}), 3.48-3.36(\mathrm{~m}, 1 \mathrm{H}), 3.30-3.14(\mathrm{~m}, 2 \mathrm{H}), 2.36(\mathrm{~s}$, $3 \mathrm{H}), 2.00(\mathrm{~d}, J=1.2 \mathrm{~Hz}, 3 \mathrm{H}), 1.85(\mathrm{~d}, J=1.2 \mathrm{~Hz}, 3 \mathrm{H}) .{ }^{13} \mathrm{C} \mathrm{NMR}\left(100 \mathrm{MHz}, \mathrm{CDCl}_{3}\right): \delta 187.8,147.8,144.6$, 142.8, 140.5, 138.0, 136.9, 136.6, 133.6, 131.7, 128.6, 127.9, 127.8, 127.5, 126.3, 126.0, 123.8, 57.0, 55.0, 36.8, 21.4, 16.4, 16.3. IR (KBr): 3057, 3025, 2923, 2855, 1634, 1481, 1447, 1399, 1374, 1317, 965, 879, 849, 748, $694 \mathrm{~cm}^{-1}$. HRMS (ESI) $\mathrm{m} / \mathrm{z}$ calculated for $\mathrm{C}_{25} \mathrm{H}_{24} \mathrm{ONa}[\mathrm{M}+\mathrm{Na}]^{+} 363.1725$, found 363.1711 .

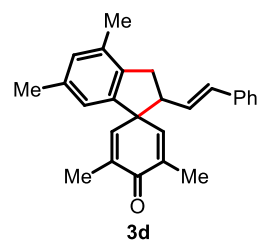

(E)-3,4',5,6'-Tetramethyl-2'-styryl-2',3'-dihydrospiro[cyclohexa[2,5]diene-1,1'-inden]-4-one (3d)

Light yellow solid (58.1 mg, 82\% yield). PE/EA $=20: 1, \mathrm{R}_{\mathrm{f}}=0.41 .{ }^{1} \mathrm{H} \mathrm{NMR}\left(400 \mathrm{MHz}, \mathrm{CDCl}_{3}\right): \delta$ 7.32-7.28 (m, 4H), 7.25-7.19 (m, 1H), $6.92(\mathrm{~s}, 1 \mathrm{H}), 6.73(\mathrm{~s}, 1 \mathrm{H}), 6.65(\mathrm{~s}, 1 \mathrm{H}), 6.56(\mathrm{~s}, 1 \mathrm{H}), 6.46(\mathrm{~d}, J=15.8$ $\mathrm{Hz}, 1 \mathrm{H}), 6.13(\mathrm{dd}, J=15.8,7.4 \mathrm{~Hz}, 1 \mathrm{H}), 3.47-3.36(\mathrm{~m}, 1 \mathrm{H}), 3.29-3.20(\mathrm{~m}, 1 \mathrm{H}), 3.12-3.02(\mathrm{~m}, 1 \mathrm{H}), 2.32(\mathrm{~s}$, $3 \mathrm{H}), 2.27$ (s, 3H), 2.03 (s, 3H), 1.87 (s, 3H). ${ }^{13} \mathrm{C} \mathrm{NMR}\left(100 \mathrm{MHz}, \mathrm{CDCl}_{3}\right): \delta 187.8,148.0,144.8,143.4$, 138.5, 137.2, 137.0, 136.5, 134.6, 133.5, 131.7, 129.9, 128.6, 127.9, 127.5, 126.3, 121.9, 57.6, 54.6, 35.1, 21.2, 19.2, 16.4, 16.3. IR (KBr): 3060, 3026, 2923, 2853, 1636, 1595, 1494, 1477, 1447, 1397, 1373, 1264 , $963,746,694 \mathrm{~cm}^{-1}$. HRMS (ESI) $\mathrm{m} / \mathrm{z}$ calculated for $\mathrm{C}_{26} \mathrm{H}_{26} \mathrm{ONa}[\mathrm{M}+\mathrm{Na}]^{+} 377.1881$, found 377.1867.

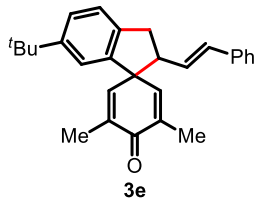

(E)-6'-(Tert-butyl)-3,5-dimethyl-2'-styryl-2',3'-dihydrospiro[cyclohexa[2,5]diene-1,1'-inden]-4-one (3e)

Light yellow solid (49.7 mg, 65\% yield). PE/EA $=20: 1, \mathrm{R}_{\mathrm{f}}=0.43 .{ }^{1} \mathrm{H}$ NMR $\left(400 \mathrm{MHz}, \mathrm{CDCl}_{3}\right): \delta$ 7.36-7.15 (m, 7H), $6.88(\mathrm{~d}, J=1.4 \mathrm{~Hz}, 1 \mathrm{H}), 6.77-6.73(\mathrm{~m}, 1 \mathrm{H}), 6.69-6.65(\mathrm{~m}, 1 \mathrm{H}), 6.46-6.39(\mathrm{~m}, 1 \mathrm{H}), 6.09$ (dd, $J=15.8,7.5 \mathrm{~Hz}, 1 \mathrm{H}), 3.51-3.37(\mathrm{~m}, 1 \mathrm{H}), 3.30-3.12(\mathrm{~m}, 2 \mathrm{H}), 2.03(\mathrm{~d}, J=1.2 \mathrm{~Hz}, 3 \mathrm{H}), 1.87(\mathrm{~d}, J=1.2$ $\mathrm{Hz}, 3 \mathrm{H}), 1.28$ (s, 9H). ${ }^{13} \mathrm{C}$ NMR $\left(100 \mathrm{MHz}, \mathrm{CDCl}_{3}\right): \delta 187.9,150.6,147.8,144.8,143.5,139.7,136.9$, 136.7, 133.6, 131.7, 128.6, 127.8, 127.5, 126.3, 125.3, 124.8, 120.7, 57.6, 55.2, 36.5, 34.8, 31.5, 16.4, 16.3. 
IR (KBr): 3059, 3026, 2961, 2925, 2868, 1636, 1492, 1448, 1397, 1384, 1375, 1262, 964, 897, 825, 750, $695 \mathrm{~cm}^{-1}$. HRMS (ESI) m/z calculated for $\mathrm{C}_{28} \mathrm{H}_{30} \mathrm{ONa}[\mathrm{M}+\mathrm{Na}]^{+} 405.2194$, found 405.2180 .

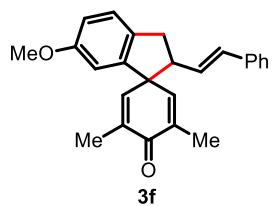

(E)-6'-Methoxy-3,5-dimethyl-2'-styryl-2',3'-dihydrospiro[cyclohexa[2,5]diene-1,1'-inden]-4-one (3f)

Yellow solid (29.9 mg, $42 \%$ yield). $\mathrm{PE} / \mathrm{EA}=20: 1, \mathrm{R}_{\mathrm{f}}=0.36 .{ }^{1} \mathrm{H} \mathrm{NMR}\left(400 \mathrm{MHz}, \mathrm{CDCl}_{3}\right): \delta 7.23-7.19$ (m, 4H), 7.18-7.10 (m, 2H), $6.86(\mathrm{~d}, J=8.0 \mathrm{~Hz}, 1 \mathrm{H}), 6.65(\mathrm{~s}, 1 \mathrm{H}), 6.57(\mathrm{~s}, 1 \mathrm{H}), 6.43-6.28(\mathrm{~m}, 2 \mathrm{H}), 6.16$ (dd, $J=16.0,7.6 \mathrm{~Hz}, 1 \mathrm{H}), 3.67(\mathrm{~s}, 3 \mathrm{H}), 3.43-3.28(\mathrm{~m}, 1 \mathrm{H}), 3.22-3.01(\mathrm{~m}, 2 \mathrm{H}), 1.94(\mathrm{~s}, 3 \mathrm{H}), 1.80(\mathrm{~s}, 3 \mathrm{H}) .{ }^{13} \mathrm{C}$ NMR $\left(100 \mathrm{MHz}, \mathrm{CDCl}_{3}\right): \delta 187.7,159.2,147.5,145.0,144.3,136.9,136.8,134.4,133.8,131.7,128.6$, 127.6, 127.5, 126.3, 125.9, 114.1, 109.1, 57.5, 55.5, 55.4, 36.1, 16.4, 16.3. IR (KBr): 3058, 3025, 2953, 2924, 2854, 1636, 1609, 1489, 1464, 1448, 1374, 1350, 1214, 964, 871, 814, 746, $694 \mathrm{~cm}^{-1}$. HRMS (ESI) $\mathrm{m} / \mathrm{z}$ calculated for $\mathrm{C}_{25} \mathrm{H}_{24} \mathrm{O}_{2} \mathrm{Na}[\mathrm{M}+\mathrm{Na}]^{+} 379.1674$, found 379.1662 .

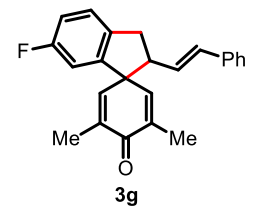

(E)-6'-Fluoro-3,5-dimethyl-2'-styryl-2',3'-dihydrospiro[cyclohexa[2,5]diene-1,1'-inden]-4-one (3g)

Light yellow solid (61.3 mg, 89\% yield). PE/EA $=20: 1, \mathrm{R}_{\mathrm{f}}=0.44 .{ }^{1} \mathrm{H} \mathrm{NMR}\left(400 \mathrm{MHz}, \mathrm{CDCl}_{3}\right): \delta$ 7.30-7.19 (m, 6H), 6.98-6.91 (m, 1H), 6.73-6.68 (m, 1H), 6.66-6.57 (m, 2H), 6.48-6.40 (m, 1H), 6.08 (dd, $J$ $=15.8,7.5 \mathrm{~Hz}, 1 \mathrm{H}), 3.51-3.40(\mathrm{~m}, 1 \mathrm{H}), 3.30-3.13(\mathrm{~m}, 2 \mathrm{H}), 2.01(\mathrm{~d}, J=1.3 \mathrm{~Hz}, 3 \mathrm{H}), 1.87(\mathrm{~d}, J=1.3 \mathrm{~Hz}$, $3 \mathrm{H}) .{ }^{13} \mathrm{C}$ NMR (100 MHz, $\left.\mathrm{CDCl}_{3}\right): \delta 187.4,162.3(\mathrm{~d}, J=246.3 \mathrm{~Hz}), 146.7,145.8(\mathrm{~d}, J=7.3 \mathrm{~Hz}), 143.6$, $137.8(\mathrm{~d}, J=2.6 \mathrm{~Hz}), 137.1,136.7,134.2,132.0,128.6,127.6,127.2,126.3,126.2(\mathrm{~d}, J=8.6 \mathrm{~Hz}), 115.0(\mathrm{~d}$, $J=22.3 \mathrm{~Hz}), 111.2(\mathrm{~d}, J=22.5 \mathrm{~Hz}), 57.3,55.4,36.2,16.4,16.3$. IR (KBr): 3059, 3026, 2924, 2853, 1635 , $1483,1448,1375,1317,1262,965,870,815,749,696 \mathrm{~cm}^{-1}$. HRMS (ESI) $\mathrm{m} / \mathrm{z}$ calculated for $\mathrm{C}_{24} \mathrm{H}_{21} \mathrm{FONa}$ $[\mathrm{M}+\mathrm{Na}]^{+} 367.1474$, found 367.1462 .

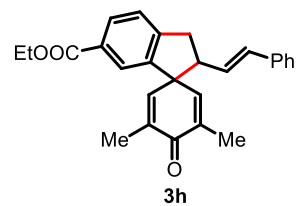

(E)-Ethyl 3,5-dimethyl-4-oxo-2'-styryl-2',3'-dihydrospiro[cyclohexa[2,5]diene-1,1'-indene]-6'-carboxylate (3h)

Light yellow solid (69.3 mg, 87\% yield). PE/EA $=20: 1, \mathrm{R}_{\mathrm{f}}=0.34 .{ }^{1} \mathrm{H} \mathrm{NMR}\left(400 \mathrm{MHz}, \mathrm{CDCl}_{3}\right): \delta$ 7.99-7.95 (m, 1H), $7.55(\mathrm{~d}, J=1.2 \mathrm{~Hz}, 1 \mathrm{H}), 7.40(\mathrm{~d}, J=7.9 \mathrm{~Hz}, 1 \mathrm{H}), 7.31-7.28(\mathrm{~m}, 3 \mathrm{H}), 7.26(\mathrm{~s}, 1 \mathrm{H}), 7.25-$ $7.20(\mathrm{~m}, 1 \mathrm{H}), 6.74-6.70(\mathrm{~m}, 1 \mathrm{H}), 6.63-6.59(\mathrm{~m}, 1 \mathrm{H}), 6.49-6.42(\mathrm{~m}, 1 \mathrm{H}), 6.08(\mathrm{dd}, J=15.8,7.5 \mathrm{~Hz}, 1 \mathrm{H})$, $4.36(\mathrm{q}, J=7.1 \mathrm{~Hz}, 2 \mathrm{H}), 3.49-3.42(\mathrm{~m}, 1 \mathrm{H}), 3.38-3.22(\mathrm{~m}, 2 \mathrm{H}), 2.03(\mathrm{~d}, J=1.3 \mathrm{~Hz}, 3 \mathrm{H}), 1.88(\mathrm{~d}, J=1.3 \mathrm{~Hz}$, $3 \mathrm{H}), 1.38(\mathrm{t}, J=7.1 \mathrm{~Hz}, 3 \mathrm{H}) .{ }^{13} \mathrm{C}$ NMR $\left(100 \mathrm{MHz}, \mathrm{CDCl}_{3}\right): \delta 187.5,166.4,147.8,146.7,144.2,143.5$, 137.2, 136.7, 134.3, 132.1, 129.9, 129.7, 128.6, 127.7, 127.0, 126.3, 125.2, 125.1, 61.1, 57.0, 54.9, 36.9, 16.4, 16.3, 14.4. IR (KBr): 3058, 3027, 2924, 2853, 1715, 1637, 1610, 1578, 1494, 1447, 1397, 1368, 1281, 964, 906, 845, 747, $695 \mathrm{~cm}^{-1}$. HRMS (ESI) $\mathrm{m} / \mathrm{z}$ calculated for $\mathrm{C}_{27} \mathrm{H}_{26} \mathrm{O}_{3} \mathrm{Na}[\mathrm{M}+\mathrm{Na}]^{+} 421.1780$, found 421.1763. 


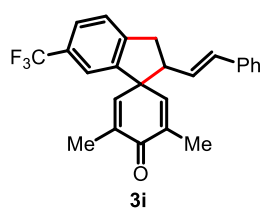

(E)-3,5-Dimethyl-2'-styryl-6'-(trifluoromethyl)-2',3'-dihydrospiro[cyclohexa[2,5]diene-1,1'-inden]-4one (3i)

Light yellow solid (59.2 mg, 75\% yield). PE/EA $=20: 1, \mathrm{R}_{\mathrm{f}}=0.39 .{ }^{1} \mathrm{H}$ NMR $\left(400 \mathrm{MHz}, \mathrm{CDCl}_{3}\right): \delta$ 7.55-7.50 (m, 1H), $7.44(\mathrm{~d}, J=7.9 \mathrm{~Hz}, 1 \mathrm{H}), 7.31-7.20(\mathrm{~m}, 5 \mathrm{H}), 7.15(\mathrm{~s}, 1 \mathrm{H}), 6.73-6.69(\mathrm{~m}, 1 \mathrm{H}), 6.62-6.59$ (m, 1H), 6.49-6.42 (m, 1H), 6.08 (dd, $J=15.8,7.5 \mathrm{~Hz}, 1 \mathrm{H}), 3.52-3.42(\mathrm{~m}, 1 \mathrm{H}), 3.39-3.22(\mathrm{~m}, 2 \mathrm{H}), 2.04(\mathrm{~d}$, $J=1.3 \mathrm{~Hz}, 3 \mathrm{H}), 1.89(\mathrm{~d}, J=1.3 \mathrm{~Hz}, 3 \mathrm{H}) .{ }^{13} \mathrm{C}$ NMR $\left(100 \mathrm{MHz}, \mathrm{CDCl}_{3}\right): \delta 187.3,146.6,146.3,144.8,143.1$, $137.4,136.6,134.5,132.3,129.8(\mathrm{q}, J=32.0 \mathrm{~Hz}), 128.6,127.7,126.8,126.3,125.6,125.2(\mathrm{q}, J=3.6 \mathrm{~Hz})$, $124.1(\mathrm{q}, J=270.6 \mathrm{~Hz}), 121.0(\mathrm{q}, J=3.7 \mathrm{~Hz}), 57.0,55.0,36.8$, 16.4, 16.3. IR (KBr): 3057, 3027, 2924, 2853, 1637, 1495, 1447, 1425, 1374, 1274, 1239, 965, 893, 832, 747, $694 \mathrm{~cm}^{-1}$. HRMS (ESI) m/z calculated for $\mathrm{C}_{25} \mathrm{H}_{21} \mathrm{~F}_{3} \mathrm{ONa}[\mathrm{M}+\mathrm{Na}]^{+} 417.1442$, found 417.1430 .

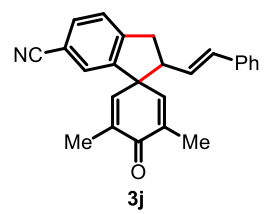

(E)-3,5-Dimethyl-4-oxo-2'-styryl-2',3'-dihydrospiro[cyclohexa[2,5]diene-1,1'-indene]-6'-carbonitrile $(3 \mathbf{j})$

Yellow solid (32.3 mg, 46\% yield). PE/EA $=20: 1, \mathrm{R}_{\mathrm{f}}=0.32 .{ }^{1} \mathrm{H} \mathrm{NMR}\left(400 \mathrm{MHz}, \mathrm{CDCl}_{3}\right): \delta 7.55(\mathrm{~d}, J$ $=7.8 \mathrm{~Hz}, 1 \mathrm{H}), 7.44(\mathrm{~d}, J=7.8 \mathrm{~Hz}, 1 \mathrm{H}), 7.30-7.26(\mathrm{~m}, 5 \mathrm{H}), 7.21(\mathrm{~s}, 1 \mathrm{H}), 6.67(\mathrm{~s}, 1 \mathrm{H}), 6.58(\mathrm{~s}, 1 \mathrm{H}), 6.46(\mathrm{~d}$, $J=15.8 \mathrm{~Hz}, 1 \mathrm{H}), 6.06(\mathrm{dd}, J=15.8,7.4 \mathrm{~Hz}, 1 \mathrm{H}), 3.48-3.42(\mathrm{~m}, 1 \mathrm{H}), 3.39-3.24(\mathrm{~m}, 2 \mathrm{H}), 2.03(\mathrm{~s}, 3 \mathrm{H}), 1.88$ (s, 3H). ${ }^{13} \mathrm{C}$ NMR $\left(100 \mathrm{MHz}, \mathrm{CDCl}_{3}\right): \delta 187.1,148.0,145.7,145.3,142.5,137.7,136.5,134.8,132.6$, 132.1, 128.6, 127.9, 127.8, 126.3, 126.2, 118.7, 111.1, 56.9, 54.7, 37.1, 16.4, 16.3. IR (KBr): 3059, 3028, 2924, 2854, 2228, 1636, 1495, 1482, 1448, 1375, 1266, 966, 902, 829, 740, $696 \mathrm{~cm}^{-1}$. HRMS (ESI) m/z calculated for $\mathrm{C}_{25} \mathrm{H}_{21} \mathrm{NONa}[\mathrm{M}+\mathrm{Na}]^{+} 374.1521$, found 374.1507.

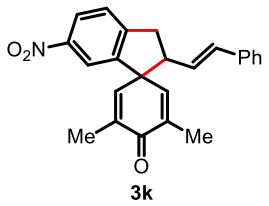

(E)-3,5-Dimethyl-6'-nitro-2'-styryl-2',3'-dihydrospiro[cyclohexa[2,5]diene-1,1'-inden]-4-one (3k)

Yellow solid (36.4 mg, $49 \%$ yield). PE/EA $=20: 1, \mathrm{R}_{\mathrm{f}}=0.30 .{ }^{1} \mathrm{H}$ NMR $\left(400 \mathrm{MHz}, \mathrm{CDCl}_{3}\right): \delta 8.17-8.13$ (m, 1H), $7.76(\mathrm{~d}, J=2.1 \mathrm{~Hz}, 1 \mathrm{H}), 7.49(\mathrm{~d}, J=8.3 \mathrm{~Hz}, 1 \mathrm{H}), 7.33-7.27(\mathrm{~m}, 4 \mathrm{H}), 7.25-7.20(\mathrm{~m}, 1 \mathrm{H}), 6.70(\mathrm{~d}, J$ $=1.4 \mathrm{~Hz}, 1 \mathrm{H}), 6.58(\mathrm{~d}, J=1.4 \mathrm{~Hz}, 1 \mathrm{H}), 6.47(\mathrm{~d}, J=15.8 \mathrm{~Hz}, 1 \mathrm{H}), 6.07(\mathrm{dd}, J=15.8,7.5 \mathrm{~Hz}, 1 \mathrm{H}), 3.56-$ $3.46(\mathrm{~m}, 1 \mathrm{H}), 3.44-3.24(\mathrm{~m}, 2 \mathrm{H}), 2.05(\mathrm{~d}, J=1.0 \mathrm{~Hz}, 3 \mathrm{H}), 1.89(\mathrm{~d}, J=1.0 \mathrm{~Hz}, 3 \mathrm{H}) .{ }^{13} \mathrm{C}$ NMR $(100 \mathrm{MHz}$, $\left.\mathrm{CDCl}_{3}\right): \delta 187.0,149.9,147.8,145.9,145.4,142.2,137.9,136.4,135.0,132.6,128.6,127.8,126.3,126.2$, 125.8, 123.7, 119.5, 56.8, 55.0, 36.8, 16.4, 16.3. IR (KBr): 3062, 3029, 2923, 2853, 1637, 1520, 1495, 1447, $1375,1345,1262,964,899,842,749,695 \mathrm{~cm}^{-1}$. HRMS (ESI) $\mathrm{m} / \mathrm{z}$ calculated for $\mathrm{C}_{24} \mathrm{H}_{21} \mathrm{NO}_{3} \mathrm{Na}[\mathrm{M}+\mathrm{Na}]^{+}$ 394.1419, found 394.1408 . 


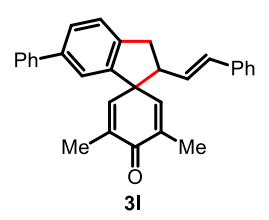

(E)-3,5-Dimethyl-6'-phenyl-2'-styryl-2',3'-dihydrospiro[cyclohexa[2,5]diene-1,1'-inden]-4-one (3I)

Yellow solid $\left(66.7 \mathrm{mg}, 83 \%\right.$ yield). PE/EA $=20: 1, \mathrm{R}_{\mathrm{f}}=0.47 .{ }^{1} \mathrm{H}$ NMR $\left(400 \mathrm{MHz}, \mathrm{CDCl}_{3}\right): \delta 7.56-$ $7.49(\mathrm{~m}, 3 \mathrm{H}), 7.46-7.40(\mathrm{~m}, 3 \mathrm{H}), 7.35(\mathrm{~d}, J=7.1 \mathrm{~Hz}, 1 \mathrm{H}), 7.33-7.29(\mathrm{~m}, 4 \mathrm{H}), 7.25-7.18(\mathrm{~m}, 1 \mathrm{H}), 7.12(\mathrm{~s}$, $1 \mathrm{H}), 6.78(\mathrm{~d}, J=1.3 \mathrm{~Hz}, 1 \mathrm{H}), 6.72(\mathrm{~s}, 1 \mathrm{H}), 6.48(\mathrm{~d}, J=15.8 \mathrm{~Hz}, 1 \mathrm{H}), 6.14(\mathrm{dd}, J=15.8,7.5 \mathrm{~Hz}, 1 \mathrm{H}), 3.54-$ $3.45(\mathrm{~m}, 1 \mathrm{H}), 3.39-3.23(\mathrm{~m}, 2 \mathrm{H}), 2.04(\mathrm{~s}, 3 \mathrm{H}), 1.90(\mathrm{~s}, 3 \mathrm{H}) .{ }^{13} \mathrm{C}$ NMR $\left(100 \mathrm{MHz}, \mathrm{CDCl}_{3}\right): \delta 187.7,147.5$, $144.4,144.3,141.7,140.8,140.7,136.9,136.8,133.9,131.9,128.8,128.6,127.6,127.5,127.4,127.2$, 127.1, 126.3, 125.6, 122.8, 57.4, 55.1, 36.6, 16.5, 16.3. IR (KBr): 3058, 3027, 2924, 2853, 1634, 1495, 1477, 1448, 1374, 1264, 964, 890, 814, 750, $697 \mathrm{~cm}^{-1}$. HRMS (ESI) $\mathrm{m} / \mathrm{z}$ calculated for $\mathrm{C}_{30} \mathrm{H}_{26} \mathrm{ONa}$ $[\mathrm{M}+\mathrm{Na}]^{+} 425.1881$, found 425.1866 .

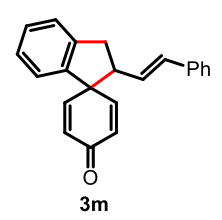

(E)-2'-Styryl-2',3'-dihydrospiro[cyclohexa[2,5]diene-1,1'-inden]-4-one (3m)

Yellow solid (39.9 mg, 67\% yield). PE/EA $=20: 1, \mathrm{R}_{\mathrm{f}}=0.33 .{ }^{1} \mathrm{H}$ NMR $\left(400 \mathrm{MHz}, \mathrm{CDCl}_{3}\right): \delta 7.37(\mathrm{~d}, J$ $=7.4 \mathrm{~Hz}, 1 \mathrm{H}), 7.32-7.27(\mathrm{~m}, 5 \mathrm{H}), 7.25-7.18(\mathrm{~m}, 2 \mathrm{H}), 7.01-6.94(\mathrm{~m}, 2 \mathrm{H}), 6.91-6.86(\mathrm{~m}, 1 \mathrm{H}), 6.55-6.45(\mathrm{~m}$, $2 \mathrm{H}), 6.26-6.21(\mathrm{~m}, 1 \mathrm{H}), 6.13(\mathrm{dd}, J=15.8,7.6 \mathrm{~Hz}, 1 \mathrm{H}), 3.55-3.46(\mathrm{~m}, 1 \mathrm{H}), 3.38-3.23(\mathrm{~m}, 2 \mathrm{H}) .{ }^{13} \mathrm{C} \mathrm{NMR}$ $\left(100 \mathrm{MHz}, \mathrm{CDCl}_{3}\right): \delta 186.6,152.3,149.5,142.7,142.3,136.5,132.4,130.7,128.6,128.5,127.8,127.7$, 127.4, 126.8, 126.3, 125.5, 124.3, 58.1, 54.9, 37.0. IR (KBr): 3060, 3026, 2925, 2852, 1663, 1611, 1494 , $1475,1449,1263,965,753,745,693 \mathrm{~cm}^{-1}$. HRMS (ESI) $\mathrm{m} / \mathrm{z}$ calculated for $\mathrm{C}_{22} \mathrm{H}_{18} \mathrm{ONa}[\mathrm{M}+\mathrm{Na}]^{+} 321.1255$, found 321.1243 .

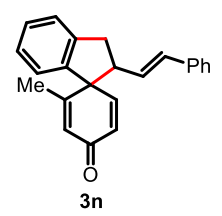

\section{(E)-2-methyl-2'-styryl-2',3'-dihydrospiro[cyclohexane-1,1'-indene]-2,5-dien-4-one (3n)}

Compound 3n was isolated by silica gel column chromatography as a diastereomeric mixture $(\mathrm{dr}=$ 2.6:1) in 70\% yield (43.7 mg). The pure major diastereomer was obtained by preparative TLC on silica gel.

White solid. PE/EA $=20: 1, \mathrm{R}_{\mathrm{f}}=0.31 .{ }^{1} \mathrm{H}$ NMR $\left(400 \mathrm{MHz}, \mathrm{CDCl}_{3}\right): \delta 7.29(\mathrm{~d}, J=7.4 \mathrm{~Hz}, 1 \mathrm{H}), 7.23-$ $7.10(\mathrm{~m}, 7 \mathrm{H}), 6.80-6.74(\mathrm{~m}, 2 \mathrm{H}), 6.41-6.33(\mathrm{~m}, 2 \mathrm{H}), 6.15-6.10(\mathrm{~m}, 1 \mathrm{H}), 6.02(\mathrm{dd}, J=15.8,7.5 \mathrm{~Hz}, 1 \mathrm{H})$, 3.67-3.54 (m, 1H), 3.31-3.16 (m, 2H), $1.89(\mathrm{~d}, J=1.0 \mathrm{~Hz}, 3 \mathrm{H}) .{ }^{13} \mathrm{C}$ NMR $\left(100 \mathrm{MHz}, \mathrm{CDCl}_{3}\right): \delta 186.6$, $159.8,149.9,143.2,142.9,136.6,132.2,131.2,128.6,128.3,127.7,127.6,127.1,126.9,126.3,125.4$, 123.8, 61.1, 52.9, 36.7, 20.3. IR (KBr): 3060, 3026, 2923, 2851, 1663, 1625, 1494, 1475, 1456, 1351, 965, $753,731,694 \mathrm{~cm}^{-1}$. HRMS (ESI) $\mathrm{m} / \mathrm{z}$ calculated for $\mathrm{C}_{23} \mathrm{H}_{20} \mathrm{ONa}[\mathrm{M}+\mathrm{Na}]^{+} 335.1412$, found 335.1404.

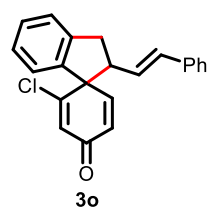




\section{(E)-2-Chloro-2'-styryl-2',3'-dihydrospiro[cyclohexa[2,5]diene-1,1'-inden]-4-one (3o)}

Compound 3o was isolated by silica gel column chromatography as a diastereomeric mixture $(\mathrm{dr}=$ 1.8:1) in $45 \%$ yield $(30.0 \mathrm{mg})$. The pure major diastereomer was obtained by preparative TLC on silica gel.

White solid. PE/EA $=20: 1, \mathrm{R}_{\mathrm{f}}=0.36 .{ }^{1} \mathrm{H}$ NMR $\left(400 \mathrm{MHz}, \mathrm{CDCl}_{3}\right): \delta 7.32(\mathrm{~d}, J=7.4 \mathrm{~Hz}, 1 \mathrm{H}), 7.29-$ $7.26(\mathrm{~m}, 1 \mathrm{H}), 7.26-7.16(\mathrm{~m}, 6 \mathrm{H}), 6.89(\mathrm{~d}, J=7.6 \mathrm{~Hz}, 1 \mathrm{H}), 6.83(\mathrm{~d}, J=10.0 \mathrm{~Hz}, 1 \mathrm{H}), 6.72(\mathrm{~d}, J=1.7 \mathrm{~Hz}$, $1 \mathrm{H}), 6.49-6.43(\mathrm{~m}, 1 \mathrm{H}), 6.20-6.15(\mathrm{~m}, 1 \mathrm{H}), 6.04(\mathrm{dd}, J=15.8,8.0 \mathrm{~Hz}, 1 \mathrm{H}), 4.03-3.90(\mathrm{~m}, 1 \mathrm{H}), 3.37-3.18$ (m, 2H). ${ }^{13} \mathrm{C}$ NMR $\left(100 \mathrm{MHz}, \mathrm{CDCl}_{3}\right): \delta 184.9,157.7,148.9,142.6,141.7,136.4,133.2,132.2,128.8$, 128.6, 127.9, 127.7, 126.4, 126.2, 125.4, 124.0, 63.3, 54.2, 36.7. IR (KBr): 3061, 3026, 2924, 2852, 1699, $1657,1595,1521,1495,753,729,694 \mathrm{~cm}^{-1}$. HRMS (ESI) $\mathrm{m} / \mathrm{z}$ calculated for $\mathrm{C}_{22} \mathrm{H}_{17} \mathrm{ClONa}[\mathrm{M}+\mathrm{Na}]^{+}$ 355.0866 , found 355.0870 .
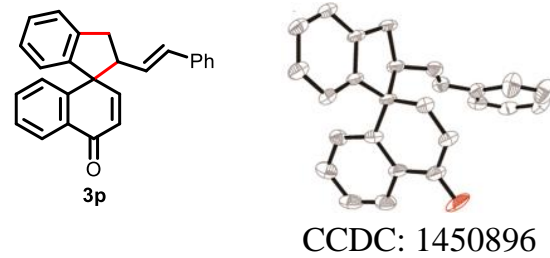

(E)-2-styryl-2,3-dihydro-4'H-spiro[indene-1,1'-naphthalen]-4'-one (3p)

Compound 3p was isolated by silica gel column chromatography as a diastereomeric mixture $(\mathrm{dr}=$ 4.5:1) in $92 \%$ yield $(64.1 \mathrm{mg})$. The pure major diastereomer was obtained by preparative TLC on silica gel and unambiguously assigned by $\mathrm{X}$-ray studies.

Yellow solid. PE/EA $=20: 1, \mathrm{R}_{\mathrm{f}}=0.43 .{ }^{1} \mathrm{H}$ NMR $\left(400 \mathrm{MHz}, \mathrm{CDCl}_{3}\right): \delta 8.25-8.21(\mathrm{~m}, 1 \mathrm{H}), 7.59-7.52$ $(\mathrm{m}, 1 \mathrm{H}), 7.47-7.39(\mathrm{~m}, 2 \mathrm{H}), 7.32(\mathrm{~d}, J=7.9 \mathrm{~Hz}, 1 \mathrm{H}), 7.30-7.26(\mathrm{~m}, 1 \mathrm{H}), 7.25-7.11(\mathrm{~m}, 6 \mathrm{H}), 6.92(\mathrm{~d}, J=$ $10.2 \mathrm{~Hz}, 1 \mathrm{H}), 6.66(\mathrm{~d}, J=7.6 \mathrm{~Hz}, 1 \mathrm{H}), 6.43(\mathrm{~d}, J=10.2 \mathrm{~Hz}, 1 \mathrm{H}), 6.20(\mathrm{~d}, J=15.9 \mathrm{~Hz}, 1 \mathrm{H}), 6.06(\mathrm{dd}, J=$ 15.8, 7.2 Hz, $1 \mathrm{H}), 3.85-3.79(\mathrm{~m}, 1 \mathrm{H}), 3.44-3.28(\mathrm{~m}, 2 \mathrm{H}) .{ }^{13} \mathrm{C} \mathrm{NMR}\left(100 \mathrm{MHz}, \mathrm{CDCl}_{3}\right): \delta$ 185.0, 149.7, 147.0, 146.4, 142.7, 136.7, 133.1, 132.8, 132.0, 128.5, 128.3, 128.0, 127.6, 127.5, 127.4, 127.2, 127.0, 126.4, 126.3, 125.1, 125.0, 59.7, 58.6, 36.7. IR (KBr): 3061, 3027, 2924, 2852, 1662, 1621, 1495, 1475, $1455,1265,965,756,737,696 \mathrm{~cm}^{-1}$. HRMS (ESI) $\mathrm{m} / \mathrm{z}$ calculated for $\mathrm{C}_{26} \mathrm{H}_{20} \mathrm{ONa}[\mathrm{M}+\mathrm{Na}]^{+} 371.1412$, found 371.1403 .

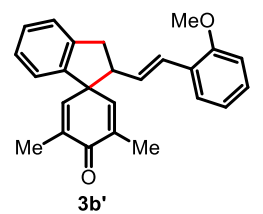

(E)-2'-(2-Methoxystyryl)-3,5-dimethyl-2',3'-dihydrospiro[cyclohexa[2,5]diene-1,1'-inden]-4-one (3b')

Light yellow solid (49.9 mg, 70\% yield). PE/EA $=10: 1, \mathrm{R}_{\mathrm{f}}=0.37 .{ }^{1} \mathrm{H}$ NMR (400 MHz, $\left.\mathrm{CDCl}_{3}\right): \delta 7.35$ (d, $J=7.4 \mathrm{~Hz}, 1 \mathrm{H}), 7.29(\mathrm{~d}, J=8.6 \mathrm{~Hz}, 2 \mathrm{H}), 7.25-7.15(\mathrm{~m}, 2 \mathrm{H}), 6.95-6.88(\mathrm{~m}, 2 \mathrm{H}), 6.88-6.83(\mathrm{~m}, 1 \mathrm{H}), 6.76$ $(\mathrm{d}, J=15.9 \mathrm{~Hz}, 2 \mathrm{H}), 6.67(\mathrm{~d}, J=1.1 \mathrm{~Hz}, 1 \mathrm{H}), 6.09(\mathrm{dd}, J=15.9,7.4 \mathrm{~Hz}, 1 \mathrm{H}), 3.83(\mathrm{~s}, 3 \mathrm{H}), 3.52-3.42(\mathrm{~m}$, $1 \mathrm{H}), 3.37-3.21(\mathrm{~m}, 2 \mathrm{H}), 2.02(\mathrm{~s}, 3 \mathrm{H}), 1.88(\mathrm{~s}, 3 \mathrm{H}) .{ }^{13} \mathrm{C} \mathrm{NMR}\left(100 \mathrm{MHz}, \mathrm{CDCl}_{3}\right): \delta 187.8,156.5,147.8$, 144.6, 143.7, 142.8, 136.6, 133.6, 128.6, 128.2, 128.0, 127.1, 126.7, 126.4, 126.0, 125.3, 124.1, 120.7, 110.9, 57.5, 55.5, 55.2, 37.0, 16.4, 16.2. IR (KBr): 3058, 3027, 2923, 2838, 1633, 1599, 1489, 1463, 1374, $1275,972,751 \mathrm{~cm}^{-1}$. HRMS (ESI) m/z calculated for $\mathrm{C}_{25} \mathrm{H}_{24} \mathrm{O}_{2} \mathrm{Na}[\mathrm{M}+\mathrm{Na}]^{+} 379.1674$, found 379.1664. 


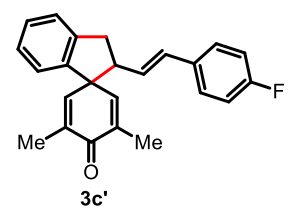

(E)-2'-(4-Fluorostyryl)-3,5-dimethyl-2',3'-dihydrospiro[cyclohexa[2,5]diene-1,1'-inden]-4-one (3c')

Light yellow solid (59.8 mg, 87\% yield). PE/EA $=20: 1, \mathrm{R}_{\mathrm{f}}=0.36 .{ }^{1} \mathrm{H} \mathrm{NMR}\left(400 \mathrm{MHz}, \mathrm{CDCl}_{3}\right): \delta$ $7.34(\mathrm{~d}, J=7.4 \mathrm{~Hz}, 1 \mathrm{H}), 7.28-7.22(\mathrm{~m}, 3 \mathrm{H}), 7.18(\mathrm{t}, J=7.4 \mathrm{~Hz}, 1 \mathrm{H}), 7.01-6.94(\mathrm{~m}, 2 \mathrm{H}), 6.91(\mathrm{~d}, J=7.4 \mathrm{~Hz}$, $1 \mathrm{H}), 6.75-6.71(\mathrm{~m}, 1 \mathrm{H}), 6.66-6.62(\mathrm{~m}, 1 \mathrm{H}), 6.40(\mathrm{~d}, J=15.7 \mathrm{~Hz}, 1 \mathrm{H}), 6.01(\mathrm{dd}, J=15.8,7.5 \mathrm{~Hz}, 1 \mathrm{H}), 3.46-$ $3.37(\mathrm{~m}, 1 \mathrm{H}), 3.33-3.18(\mathrm{~m}, 2 \mathrm{H}), 2.01(\mathrm{~d}, J=1.3 \mathrm{~Hz}, 3 \mathrm{H}), 1.87(\mathrm{~d}, J=1.3 \mathrm{~Hz}, 3 \mathrm{H}) .{ }^{13} \mathrm{C}$ NMR $(100 \mathrm{MHz}$, $\left.\mathrm{CDCl}_{3}\right): \delta 187.7,162.3(\mathrm{~d}, J=249.0 \mathrm{~Hz}), 147.5,144.3,143.5,142.5,136.8,133.8,133.0(\mathrm{~d}, J=3.3 \mathrm{~Hz})$, 130.6, 128.1, $127.8(\mathrm{~d}, J=8.0 \mathrm{~Hz}), 127.4(\mathrm{~d}, J=2.1 \mathrm{~Hz}), 127.2,125.3,124.1,115.5(\mathrm{~d}, J=21.5 \mathrm{~Hz}), 57.4$, 54.8, 36.9, 16.4, 16.3. IR (KBr): 3054, 3028, 2923, 2851, 1636, 1601, 1508, 1457, 1374, 1264, 966, 815, $749 \mathrm{~cm}^{-1}$. HRMS (ESI) $\mathrm{m} / \mathrm{z}$ calculated for $\mathrm{C}_{24} \mathrm{H}_{21} \mathrm{FONa}[\mathrm{M}+\mathrm{Na}]^{+} 367.1474$, found 367.1462.

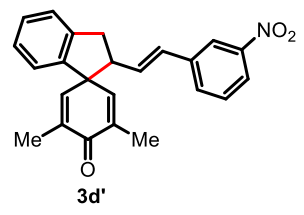

(E)-3,5-Dimethyl-2'-(3-nitrostyryl)-2',3'-dihydrospiro[cyclohexa[2,5]diene-1,1'-inden]-4-one (3d')

Yellow solid (37.1 mg, 50\% yield). PE/EA $=20: 1, \mathrm{R}_{\mathrm{f}}=0.29 .{ }^{1} \mathrm{H} \mathrm{NMR}\left(400 \mathrm{MHz}, \mathrm{CDCl}_{3}\right): \delta 8.13(\mathrm{~s}$, $1 \mathrm{H}), 8.09-8.04(\mathrm{~m}, 1 \mathrm{H}), 7.59(\mathrm{~d}, J=7.7 \mathrm{~Hz}, 1 \mathrm{H}), 7.46(\mathrm{t}, J=7.9 \mathrm{~Hz}, 1 \mathrm{H}), 7.36(\mathrm{~d}, J=7.4 \mathrm{~Hz}, 1 \mathrm{H}), 7.29(\mathrm{~d}$, $J=7.4 \mathrm{~Hz}, 1 \mathrm{H}), 7.20(\mathrm{t}, J=7.5 \mathrm{~Hz}, 1 \mathrm{H}), 6.92(\mathrm{~d}, J=7.5 \mathrm{~Hz}, 1 \mathrm{H}), 6.74(\mathrm{~s}, 1 \mathrm{H}), 6.66(\mathrm{~s}, 1 \mathrm{H}), 6.50(\mathrm{~d}, J=$ $15.8 \mathrm{~Hz}, 1 \mathrm{H}), 6.25(\mathrm{dd}, J=15.8,7.7 \mathrm{~Hz}, 1 \mathrm{H}), 3.52-3.41(\mathrm{~m}, 1 \mathrm{H}), 3.35-3.23(\mathrm{~m}, 2 \mathrm{H}), 2.02(\mathrm{~s}, 3 \mathrm{H}), 1.87(\mathrm{~s}$, $3 \mathrm{H}) .{ }^{13} \mathrm{C}$ NMR $\left(100 \mathrm{MHz}, \mathrm{CDCl}_{3}\right): \delta 187.5,148.6,147.2,143.8,143.3,142.2,138.6,137.0,134.0,132.0$, 131.1, 129.8, 129.5, 128.2, 127.3, 125.4, 124.1, 122.2, 121.0, 57.4, 54.8, 36.8, 16.4, 16.3. IR (KBr): 3061, 3030, 2924, 2853, 1636, 1528, 1475, 1457, 1374, 1265, 965, 875, 803, $761 \mathrm{~cm}^{-1}$. HRMS (ESI) m/z calculated for $\mathrm{C}_{24} \mathrm{H}_{21} \mathrm{NO}_{3} \mathrm{Na}[\mathrm{M}+\mathrm{Na}]^{+}$394.1419, found 394.1406.

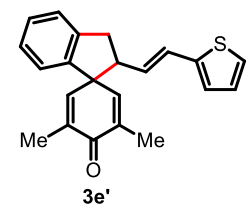

(E)-3,5-Dimethyl-2' -(2-(thiophen-2-yl)vinyl)-2',3'-dihydrospiro[cyclohexa[2,5]diene-1,1'-inden]-4-one $\left(3 \mathrm{e}^{\prime}\right)$

Yellow solid (53.8 mg, 81\% yield). PE/EA $=20: 1, \mathrm{R}_{\mathrm{f}}=0.43 .{ }^{1} \mathrm{H}$ NMR $\left(400 \mathrm{MHz}, \mathrm{CDCl}_{3}\right): \delta 7.34(\mathrm{~d}$, $J=7.4 \mathrm{~Hz}, 1 \mathrm{H}), 7.29-7.23(\mathrm{~m}, 1 \mathrm{H}), 7.18(\mathrm{t}, J=7.3 \mathrm{~Hz}, 1 \mathrm{H}), 7.12(\mathrm{~d}, J=5.0 \mathrm{~Hz}, 1 \mathrm{H}), 6.97-6.86(\mathrm{~m}, 3 \mathrm{H})$, , $6.71(\mathrm{~s}, 1 \mathrm{H}), 6.64(\mathrm{~s}, 1 \mathrm{H}), 6.56(\mathrm{~d}, J=15.6 \mathrm{~Hz}, 1 \mathrm{H}), 5.95(\mathrm{dd}, J=15.6,7.6 \mathrm{~Hz}, 1 \mathrm{H}), 3.46-3.34(\mathrm{~m}, 1 \mathrm{H})$, 3.33-3.15 (m, 2H), $2.02(\mathrm{~d}, J=0.9 \mathrm{~Hz}, 3 \mathrm{H}), 1.88(\mathrm{~d}, J=0.9 \mathrm{~Hz}, 3 \mathrm{H}) .{ }^{13} \mathrm{C}$ NMR $\left(100 \mathrm{MHz}, \mathrm{CDCl}_{3}\right): \delta 187.7$, 147.4, 144.1, 143.5, 142.5, 141.9, 136.8, 133.9, 128.1, 127.4, 127.3, 127.2, 125.4, 125.3, 124.9, 124.1, 57.4, 54.8, 36.9, 16.4, 16.2. IR (KBr): 3068, 3035, 2923, 2850, 1636, 1601, 1475, 1456, 1398, 1374, 1265, 954, 933, 874, 760, 738, $700 \mathrm{~cm}^{-1}$. HRMS (ESI) $\mathrm{m} / \mathrm{z}$ calculated for $\mathrm{C}_{22} \mathrm{H}_{20} \mathrm{SONa}[\mathrm{M}+\mathrm{Na}]^{+} 355.1133$, found 355.1119. 


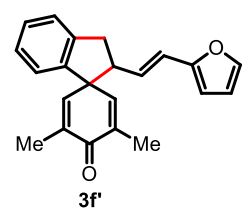

(E)-2'-(2-(Furan-2-yl)vinyl)-3,5-dimethyl-2',3'-dihydrospiro[cyclohexa[2,5]diene-1,1'-inden]-4-one (3f')

Yellow solid (50.1 mg, 79\% yield). $\mathrm{PE} / \mathrm{EA}=20: 1, \mathrm{R}_{\mathrm{f}}=0.44 .{ }^{1} \mathrm{H} \mathrm{NMR}\left(400 \mathrm{MHz}, \mathrm{CDCl}_{3}\right): \delta 7.35-7.30$ $(\mathrm{m}, 2 \mathrm{H}), 7.24(\mathrm{~d}, J=7.4 \mathrm{~Hz}, 1 \mathrm{H}), 7.17(\mathrm{t}, J=7.4 \mathrm{~Hz}, 1 \mathrm{H}), 6.90(\mathrm{~d}, J=7.5 \mathrm{~Hz}, 1 \mathrm{H}), 6.71(\mathrm{~s}, 1 \mathrm{H}), 6.64(\mathrm{~s}$, $1 \mathrm{H}), 6.37-6.32(\mathrm{~m}, 1 \mathrm{H}), 6.25(\mathrm{~d}, J=15.9 \mathrm{~Hz}, 1 \mathrm{H}), 6.17(\mathrm{~d}, J=3.2 \mathrm{~Hz}, 1 \mathrm{H}), 6.04(\mathrm{dd}, J=15.8,7.7 \mathrm{~Hz}, 1 \mathrm{H})$, 3.42-3.36 (m, 1H), 3.29-3.17 (m, 2H), $2.01(\mathrm{~d}, J=0.9 \mathrm{~Hz}, 3 \mathrm{H}), 1.87(\mathrm{~d}, J=0.9 \mathrm{~Hz}, 3 \mathrm{H}) .{ }^{13} \mathrm{C}$ NMR $(100$ $\left.\mathrm{MHz}, \mathrm{CDCl}_{3}\right): \delta 187.8,152.2,147.5,144.2,143.6,142.5,141.8,136.7,133.8,128.0,127.1,126.3,125.3$, 124.0, 120.3, 111.2, 107.6, 57.3, 54.9, 37.0, 16.4, 16.3. IR (KBr): 3065, 3033, 2923, 2851, 1635, 1489, 1474, 1457, 1398, 1374, 1261, 960, 933, 883, 763, $750 \mathrm{~cm}^{-1}$. HRMS (ESI) m/z calculated for $\mathrm{C}_{22} \mathrm{H}_{20} \mathrm{O}_{2} \mathrm{Na}$ $[\mathrm{M}+\mathrm{Na}]^{+} 339.1361$, found 339.1349 .

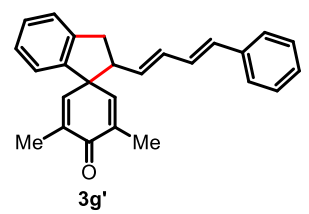

3,5-Dimethyl-2'-((1E,3E)-4-phenylbuta-1,3-dien-1-yl)-2',3'-dihydrospiro[cyclohexa[2,5]diene-1,1'inden]-4-one (3g')

Light yellow solid (46.5 mg, 66\% yield). $\mathrm{PE} / \mathrm{EA}=20: 1, \mathrm{R}_{\mathrm{f}}=0.42 .{ }^{1} \mathrm{H} \mathrm{NMR}\left(400 \mathrm{MHz}, \mathrm{CDCl}_{3}\right): \delta$ 7.39-7.28 (m, 6H), 7.24-7.14 (m, 2H), $6.90(\mathrm{~d}, J=7.5 \mathrm{~Hz}, 1 \mathrm{H}), 6.73-6.59(\mathrm{~m}, 2 \mathrm{H}), 6.62(\mathrm{~s}, 1 \mathrm{H}), 6.49(\mathrm{~d}, J$ $=15.7 \mathrm{~Hz}, 1 \mathrm{H}), 6.31-6.22(\mathrm{~m}, 1 \mathrm{H}), 5.72(\mathrm{dd}, \mathrm{J}=15.1,7.4 \mathrm{~Hz}, 1 \mathrm{H}), 3.45-3.33(\mathrm{~m}, 1 \mathrm{H}), 3.31-3.13(\mathrm{~m}, 2 \mathrm{H})$, $2.02(\mathrm{~s}, 3 \mathrm{H}), 1.88(\mathrm{~s}, 3 \mathrm{H}) .{ }^{13} \mathrm{C} \mathrm{NMR}\left(100 \mathrm{MHz} \mathrm{CDCl}_{3}\right): \delta 187.8,147.6,144.4,143.6,142.6,137.2,136.7$, 133.7, 132.3, 131.9, 131.8, 128.6, 128.5, 128.0, 127.5, 127.1, 126.3, 125.3, 124.0, 57.3, 54.8, 36.9, 16.4, 16.3. IR (KBr): 3055, 3023, 2923, 2851, 1633, 1498, 1477, 1447, 1375, 1262, 966, 753, $749,692 \mathrm{~cm}^{-1}$. HRMS (ESI) m/z calculated for $\mathrm{C}_{26} \mathrm{H}_{24} \mathrm{ONa}[\mathrm{M}+\mathrm{Na}]^{+} 375.1725$, found 375.1712 .

\section{Preliminary asymmetric studies:}

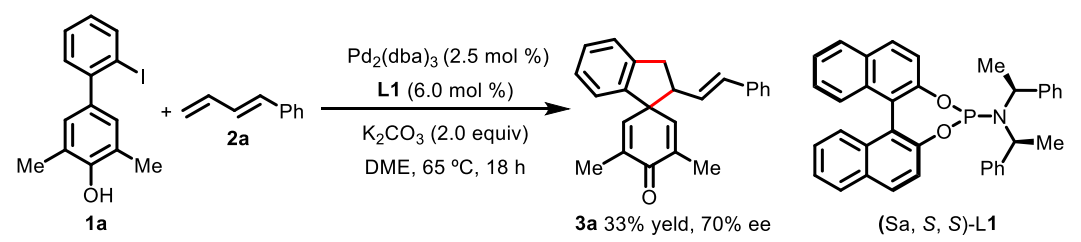

In a glovebox, a $5.0 \mathrm{~mL}$ vial equipped with a stir bar was charged with $\operatorname{Pd}_{2}(\mathrm{dba})_{3}(2.3 \mathrm{mg}, 0.0025$ mmol), phosphoramidite ligand $\mathbf{L 1}(3.2 \mathrm{mg}, 0.006 \mathrm{mmol}), \mathrm{K}_{2} \mathrm{CO}_{3}(27.6 \mathrm{mg}, 0.2 \mathrm{mmol})$ and DME (0.6 mL) was then added. After the catalyst mixture was stirred at room temperature for 10 min, substrate 1a (32.4 $\mathrm{mg}, 0.1 \mathrm{mmol})$ and 1,3-diene $\mathbf{2 a}(26.0 \mathrm{mg}, 0.2 \mathrm{mmol})$ were sequentially added. The vial was sealed with a Teflon screw cap and the reaction mixture was heated at $65^{\circ} \mathrm{C}$ for $18 \mathrm{~h}$. The crude reaction mixture was then subjected to a silica gel column to afford the desired product 3a. $33 \%$ yield. $70 \%$ ee, $[\alpha]_{\mathrm{D}}{ }^{20}=+11.9$ $\left(\mathrm{c}=0.5, \mathrm{CHCl}_{3}\right)$. The ee of compound 3a was determined by HPLC using an IB column $(n-\mathrm{hexane} / i$-PrOH $=85 / 15$, flow rate $=0.5 \mathrm{~mL} / \mathrm{min}, \mathrm{t}_{\text {major }}=10.4 \mathrm{~min}, \mathrm{t}_{\text {minor }}=12.5 \mathrm{~min}$ ). 

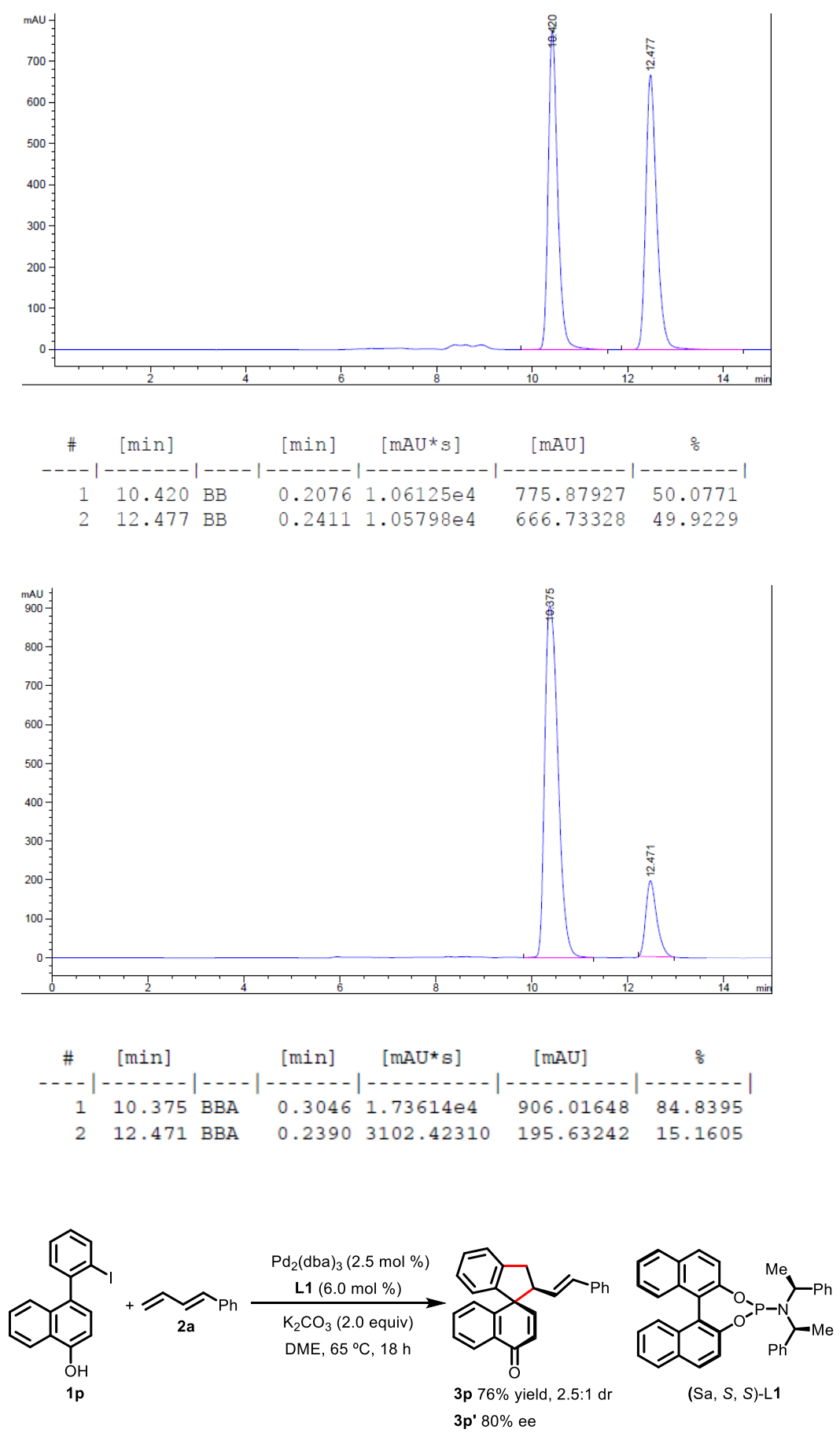

In a glovebox, a $5.0 \mathrm{~mL}$ vial equipped with a stir bar was charged with $\mathrm{Pd}_{2}(\mathrm{dba})_{3}(2.3 \mathrm{mg}, 0.0025$ mmol), phosphoramidite ligand $\mathbf{L 1}(3.2 \mathrm{mg}, 0.006 \mathrm{mmol}), \mathrm{K}_{2} \mathrm{CO}_{3}(27.6 \mathrm{mg}, 0.2 \mathrm{mmol})$ and DME $(0.6 \mathrm{~mL})$ was then added. After the catalyst mixture was stirred at room temperature for $10 \mathrm{~min}$, substrate $\mathbf{1 p}$ (34.6 $\mathrm{mg}, 0.1 \mathrm{mmol})$ and 1,3 -diene $2 \mathbf{a}(26.0 \mathrm{mg}, 0.2 \mathrm{mmol})$ were sequentially added. The vial was sealed with a Teflon screw cap and the reaction mixture was heated at $65^{\circ} \mathrm{C}$ for $18 \mathrm{~h}$. The crude reaction mixture was then subjected to a silica gel column to afford the desired product $\mathbf{3 p}$ as a diastereomeric mixture $(\mathrm{dr}=$ 2.5:1) in 76\% yield. The pure major diastereomer 3p' was obtained by preparative TLC on silica gel. 80\% ee, $[\alpha]_{\mathrm{D}}{ }^{13}=+33.1 \quad\left(\mathrm{c}=0.5, \mathrm{CHCl}_{3}\right)$. The ee of the major diastereomer $\mathbf{3} \mathbf{p}^{\prime}$ was determined by HPLC using an IB column $\left(n\right.$-hexane $/ i$-PrOH $=85 / 15$, flow rate $\left.=0.5 \mathrm{~mL} / \mathrm{min}, \mathrm{t}_{\text {major }}=16.8 \mathrm{~min}, \mathrm{t}_{\text {minor }}=14.8 \mathrm{~min}\right)$. 

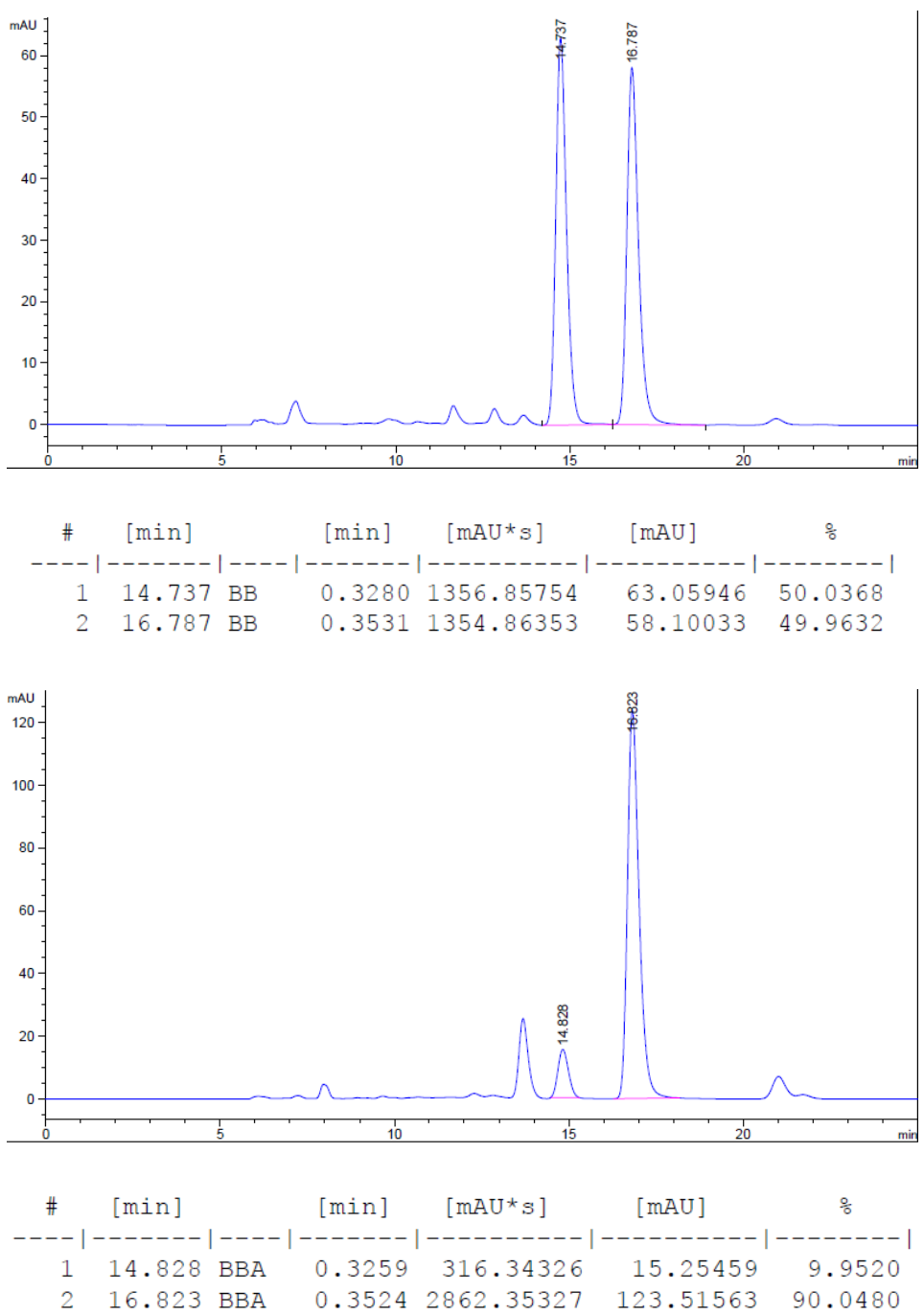

\section{E. References:}

(1) Yang, L.; Zheng, H.; Luo, L.; Nan, J.; Liu, J.; Wang, Y.; Luan, X. J. Am. Chem. Soc. 2015, 137, 4876.

(2) Anton, L.; Kilian, M. Chem. Eur. J. 2012, 18, 2212.

(3) Timsina, Y. N.; Souvagya, B.; Rajanbabu, T. V. J. Am. Chem. Soc. 2014, 136, 6215.

(4) Yildizhan, S.; Schulz, S. Synlett 2011, 2831.

(5) Lv, J.; Liu, Q.; Tang, J.; Franc, P.; Kristof, K. Tetrahedron Lett., 2012, 53, 5248. 
F. NMR spectra:
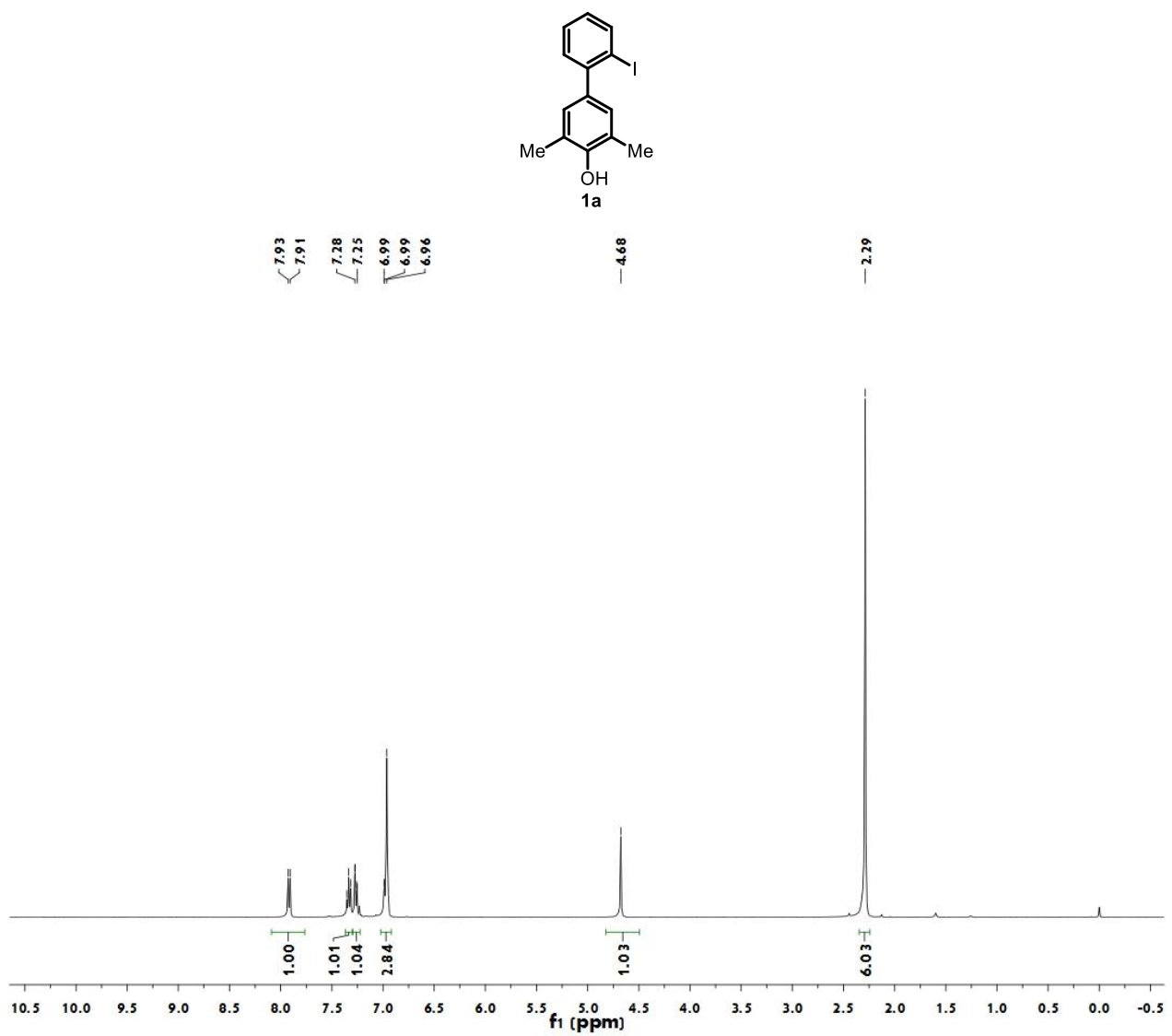

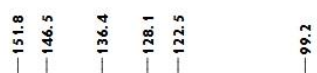

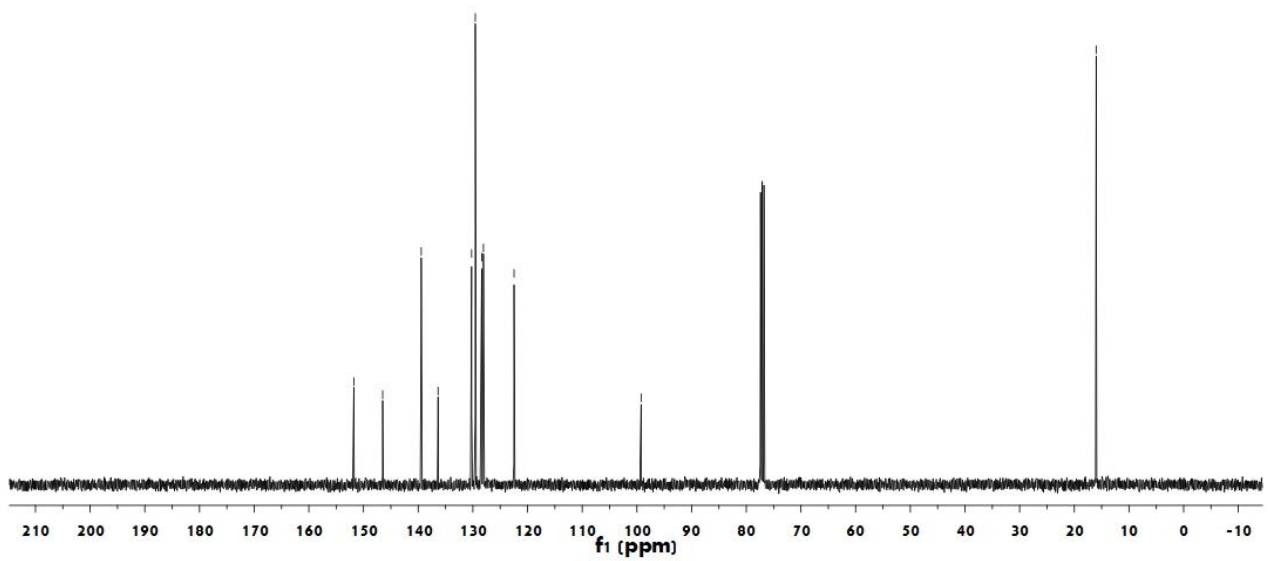




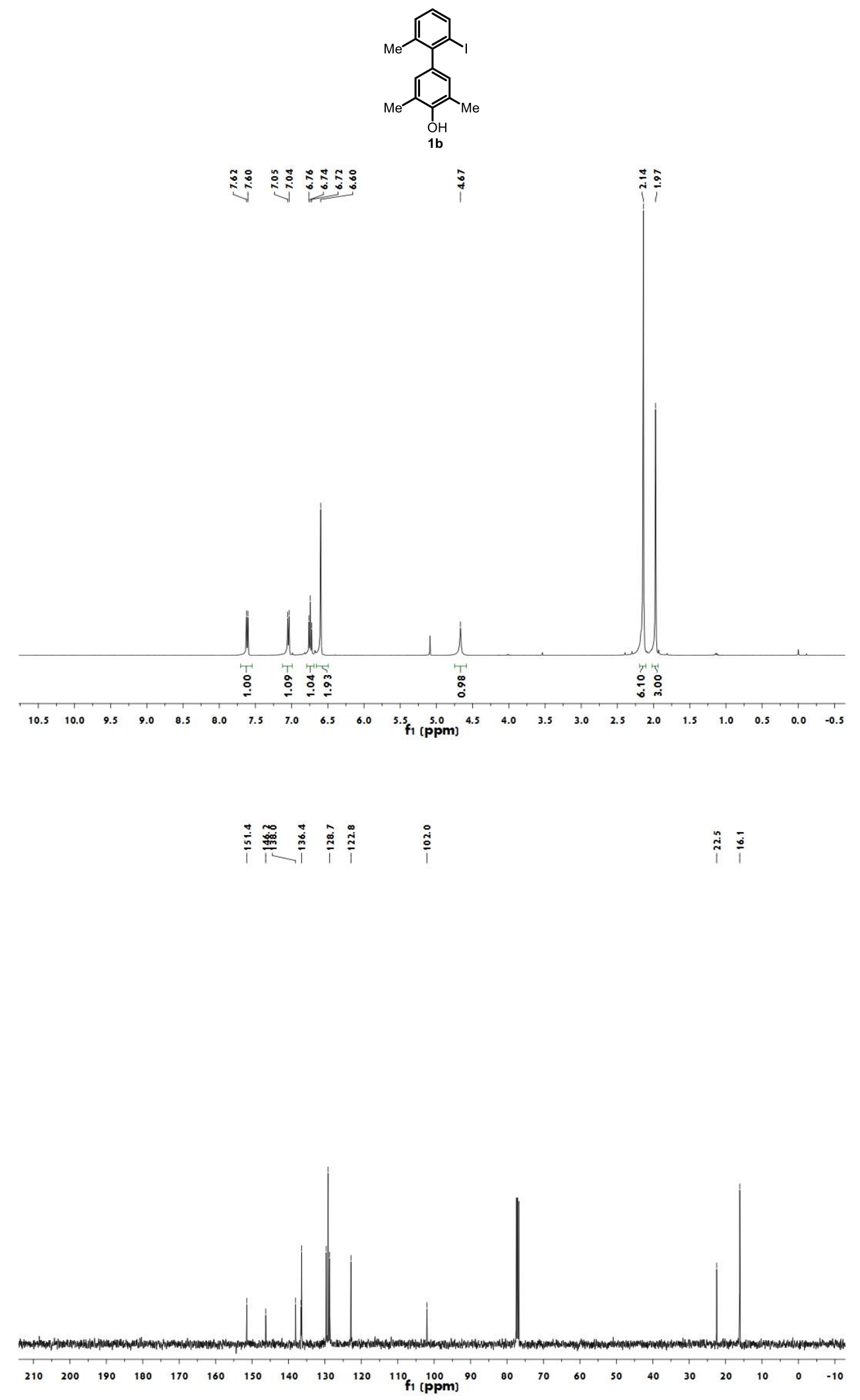




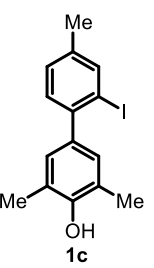

i $\quad \stackrel{n}{i} \stackrel{n}{i}$

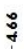

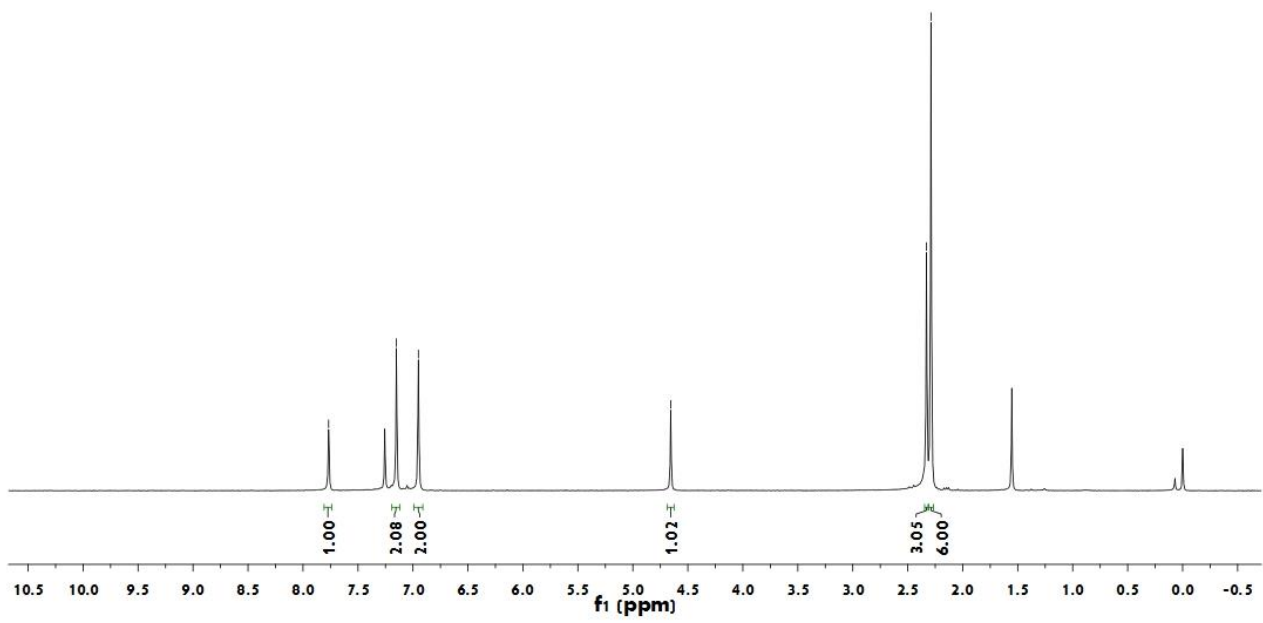

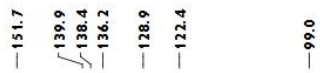

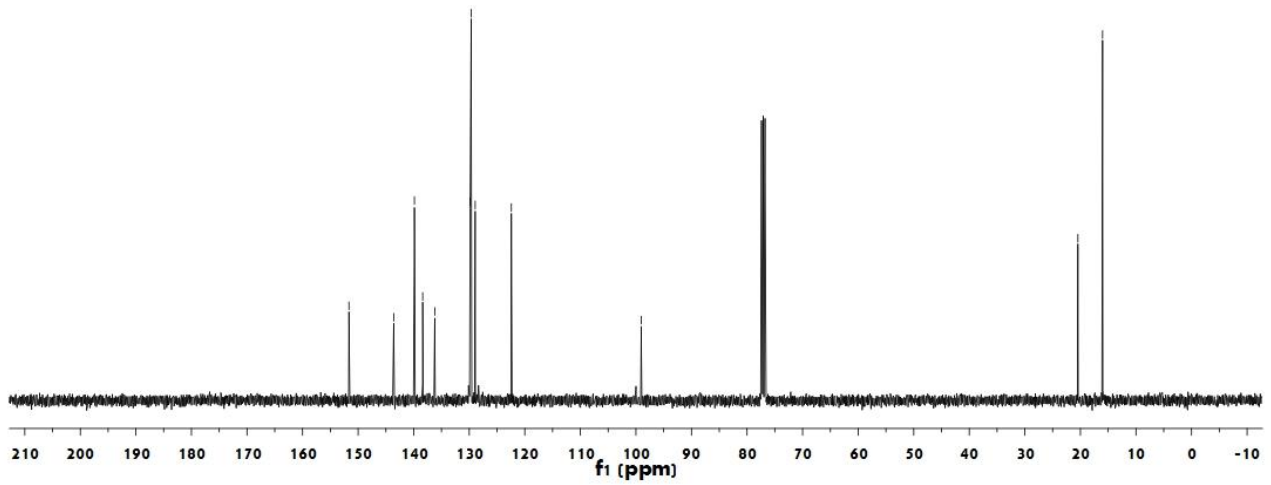



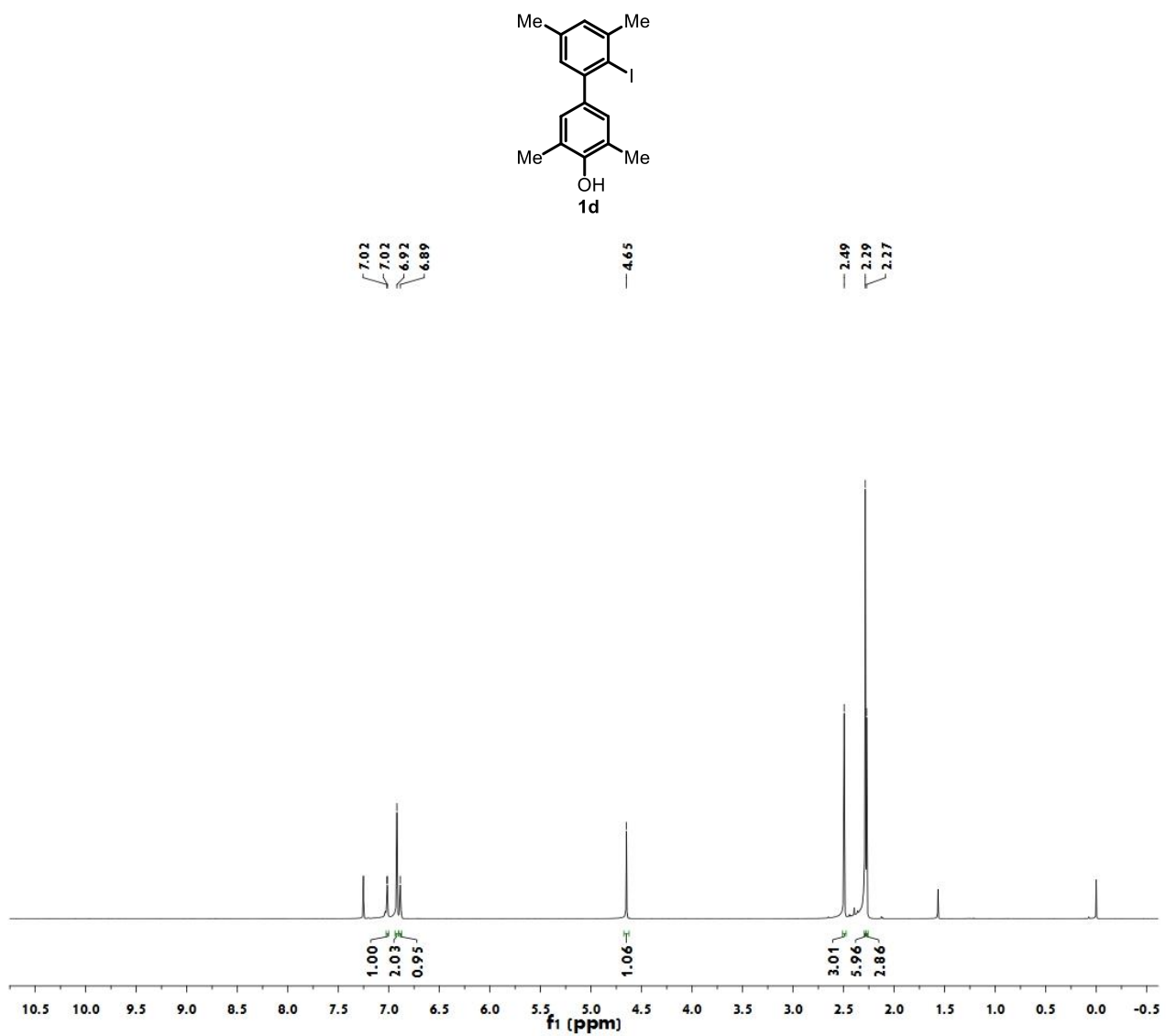

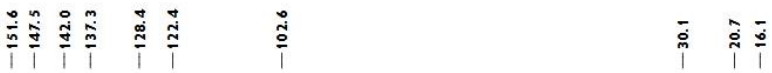

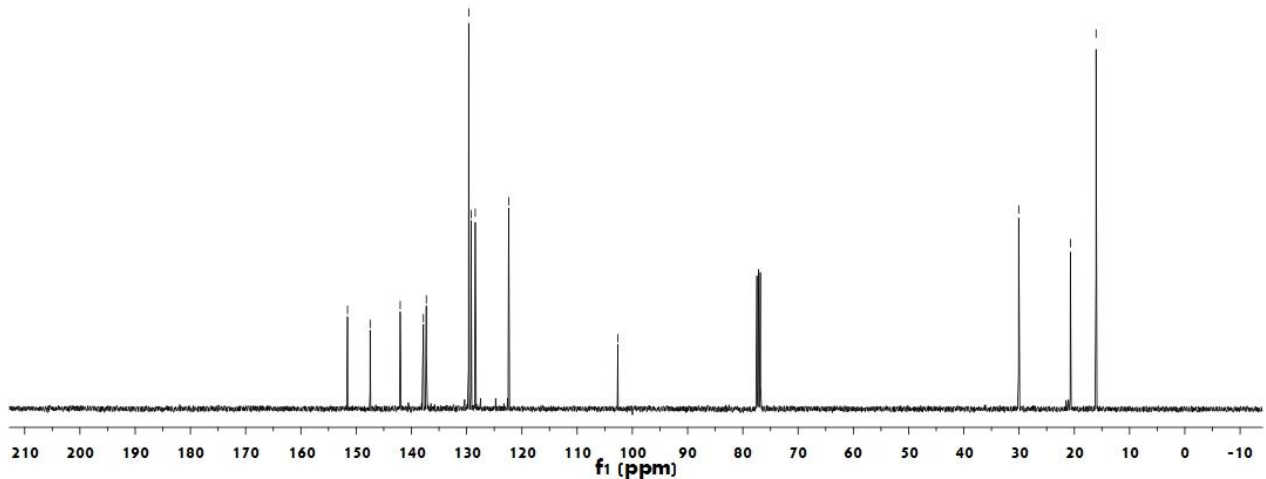



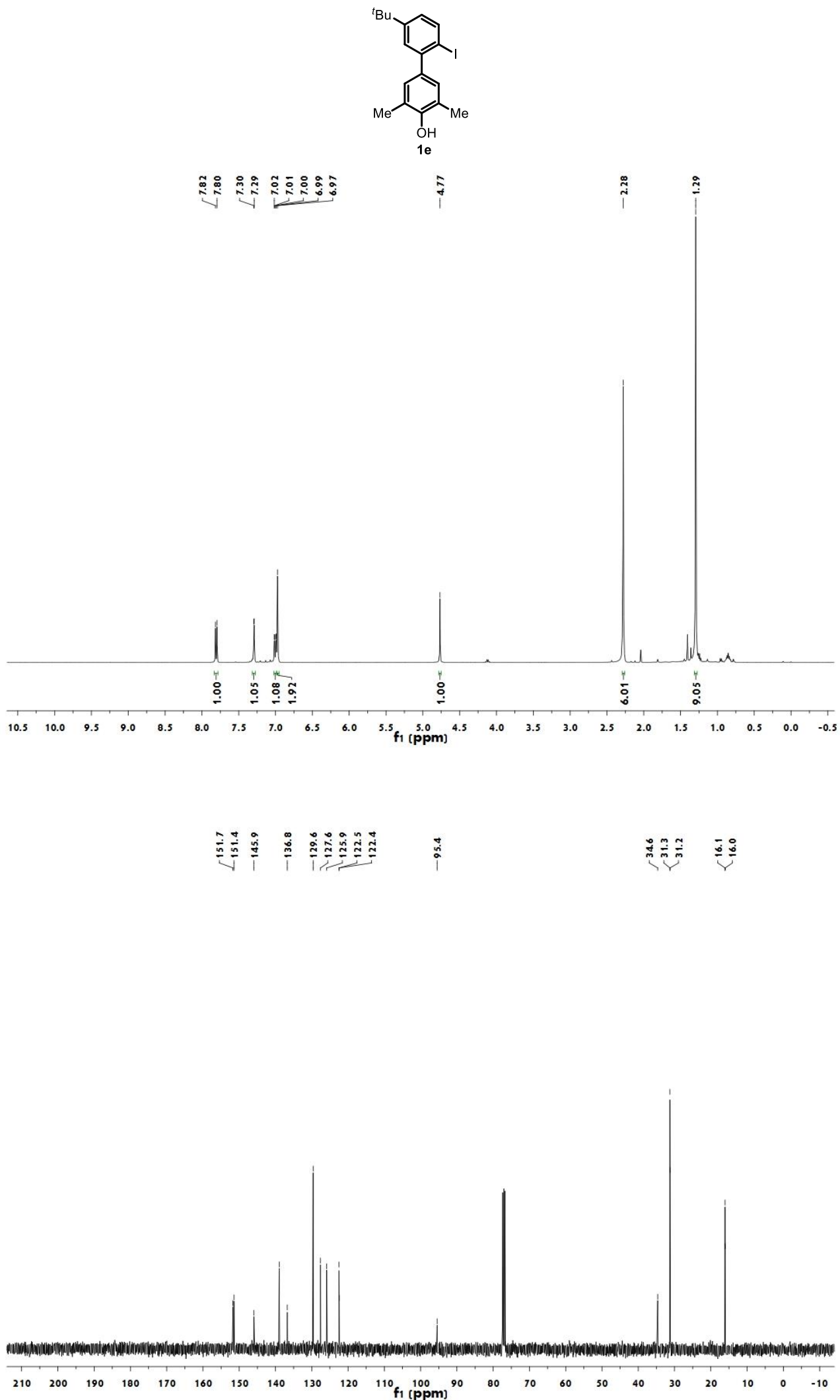


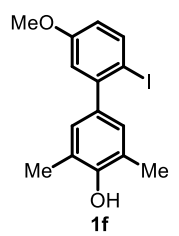

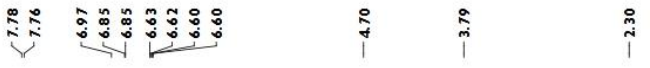

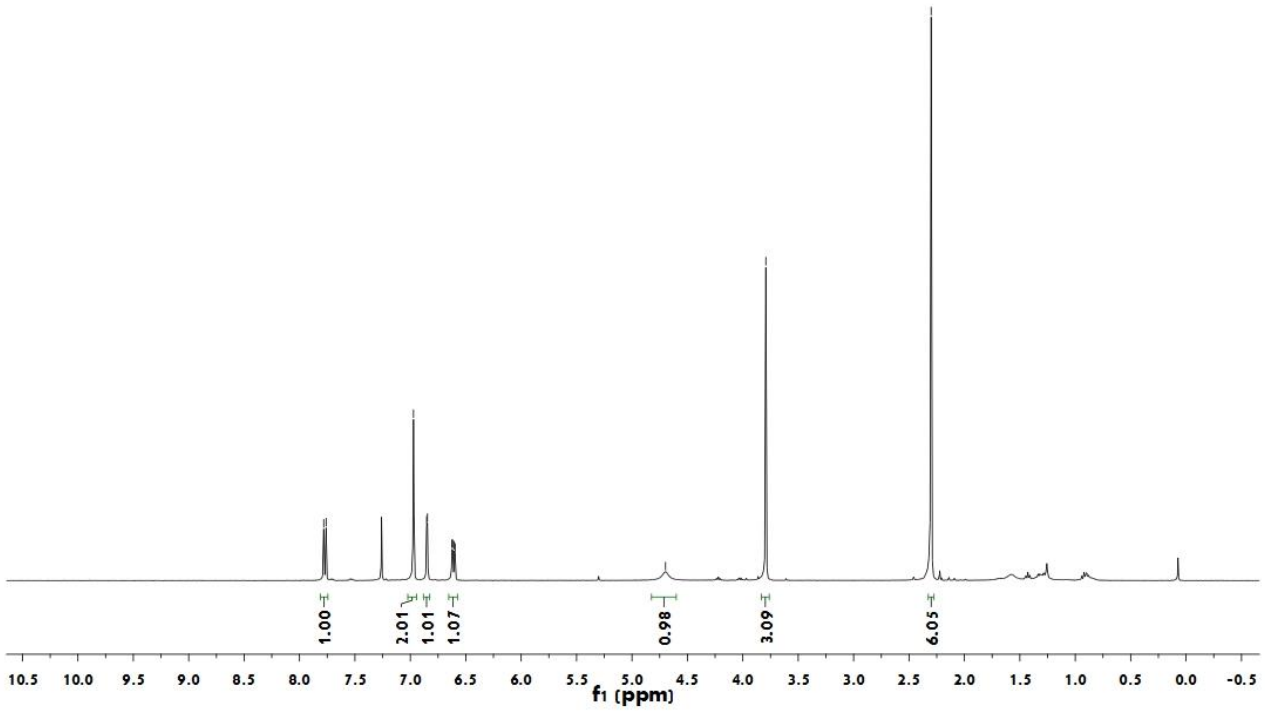

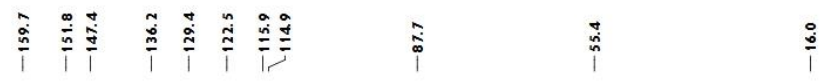

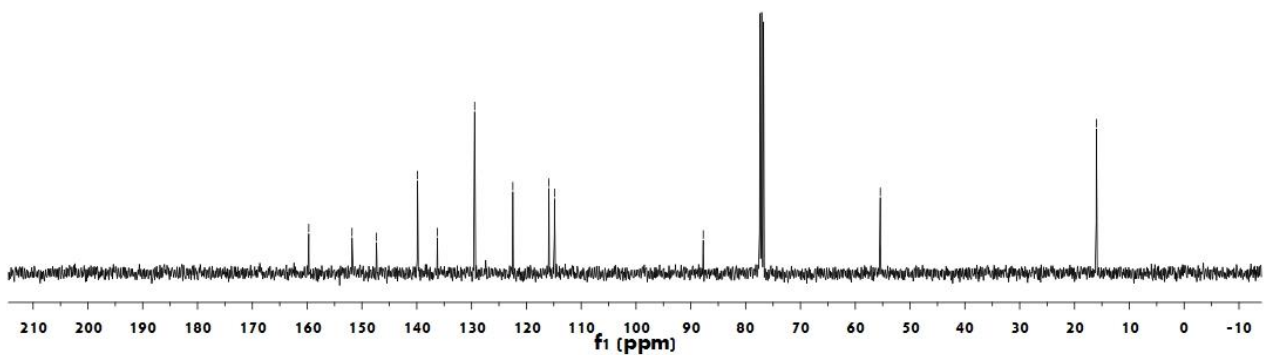




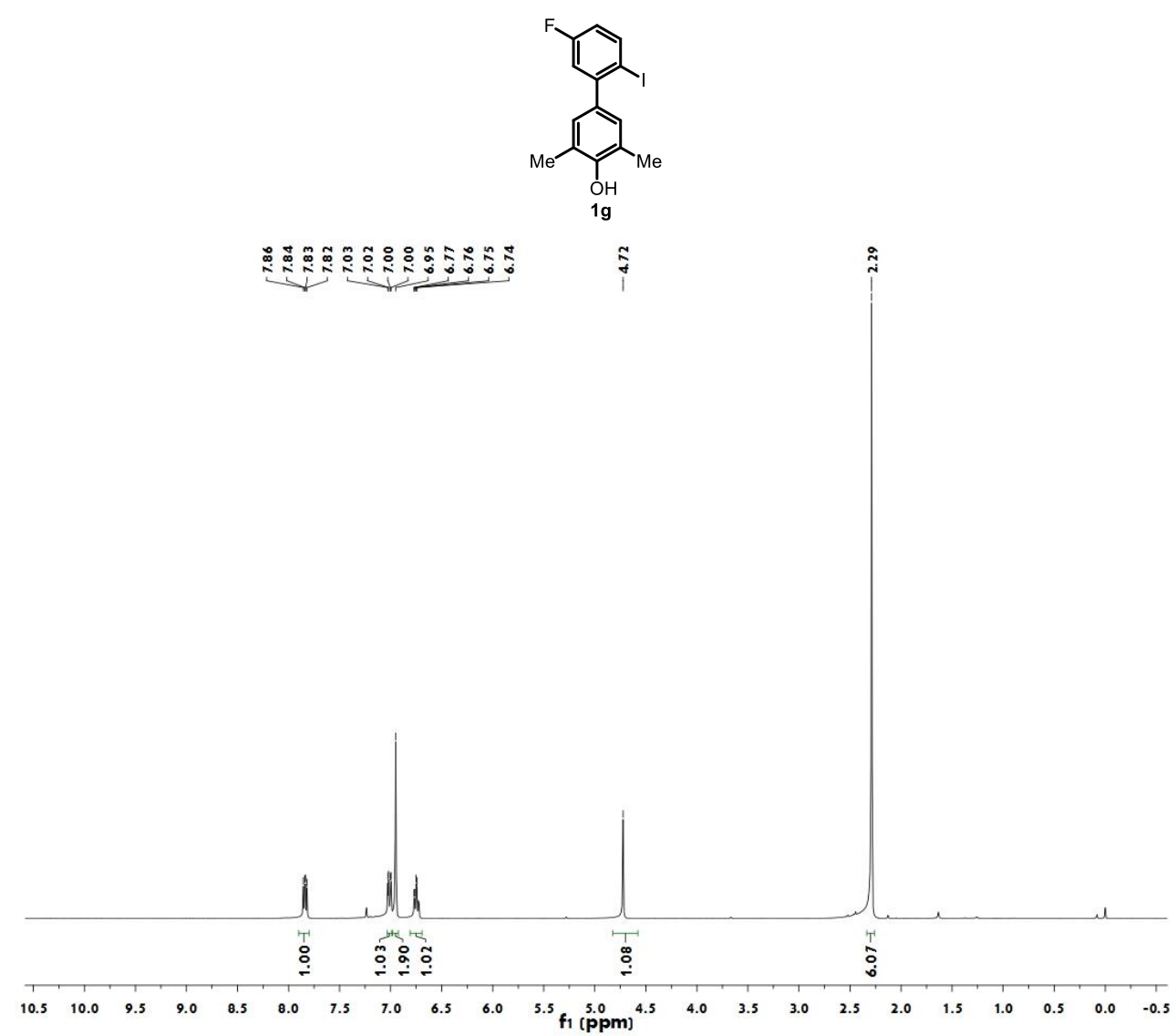

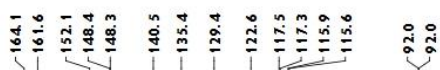

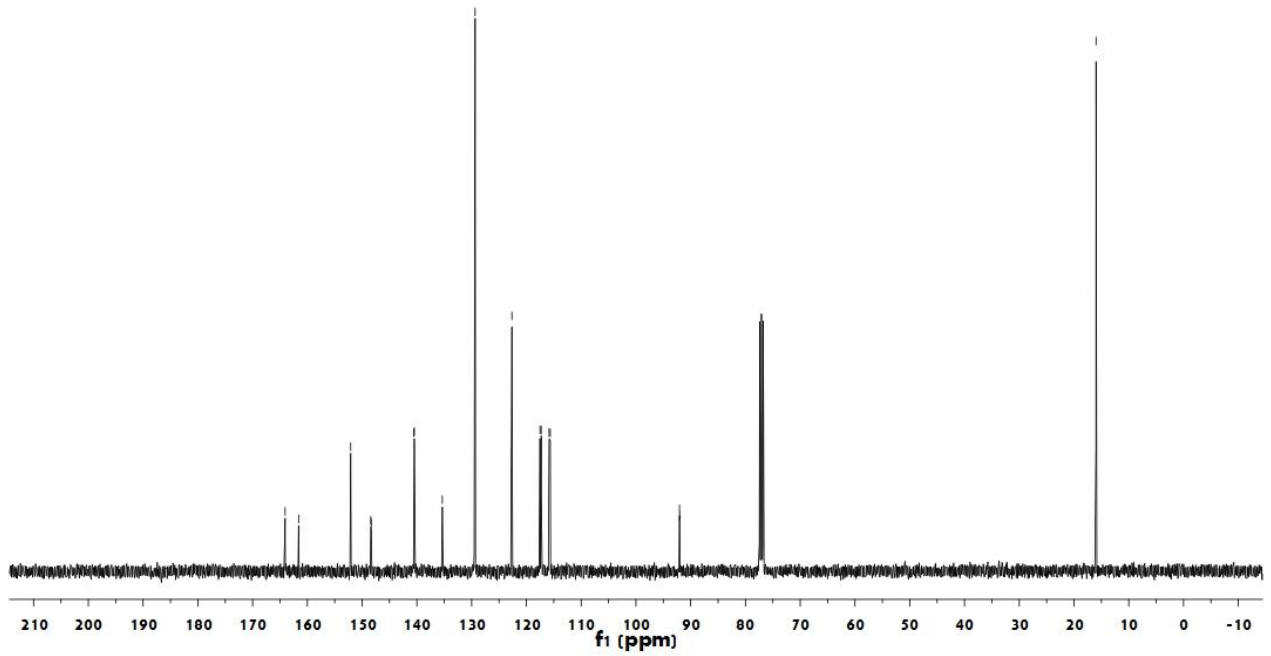



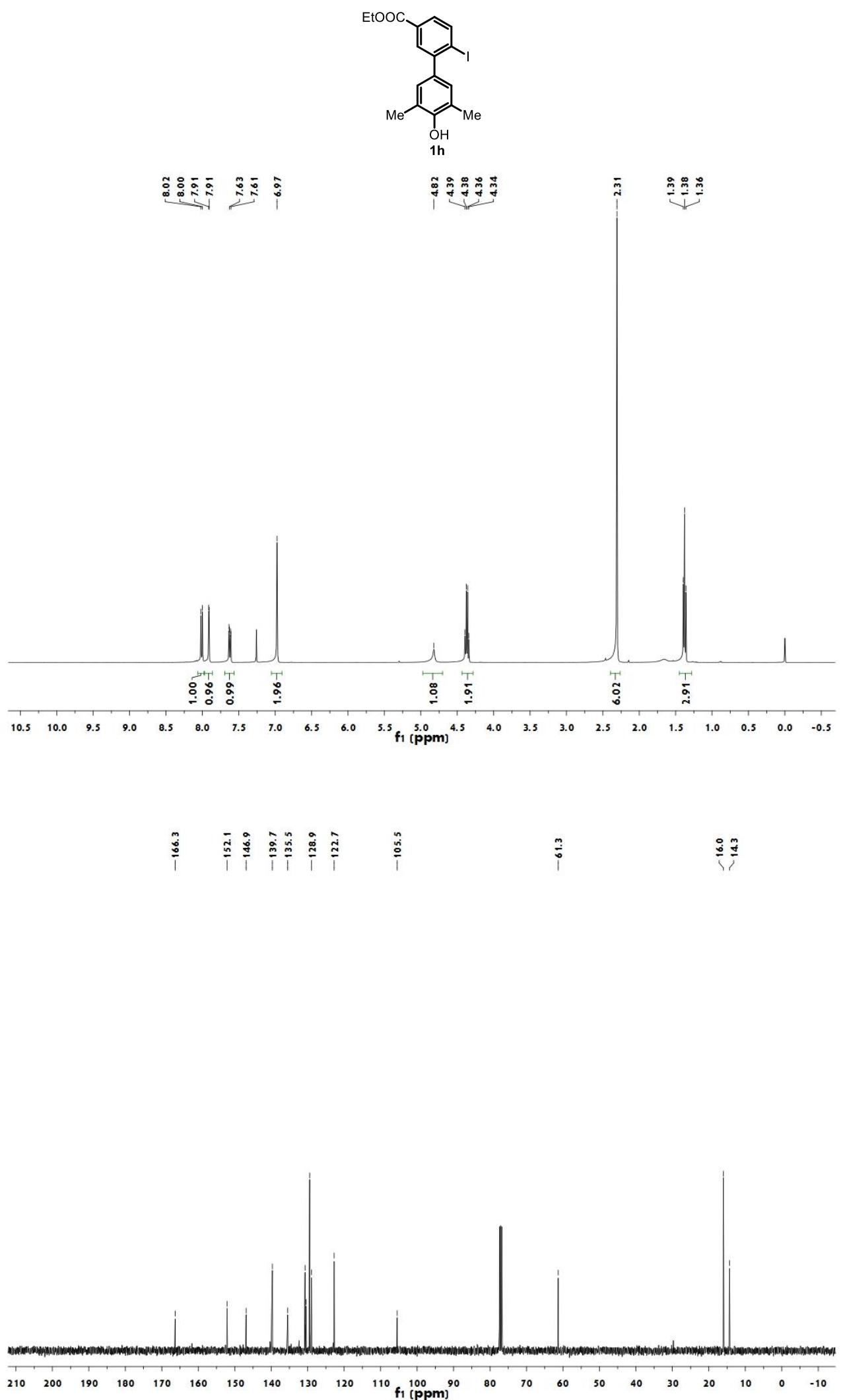

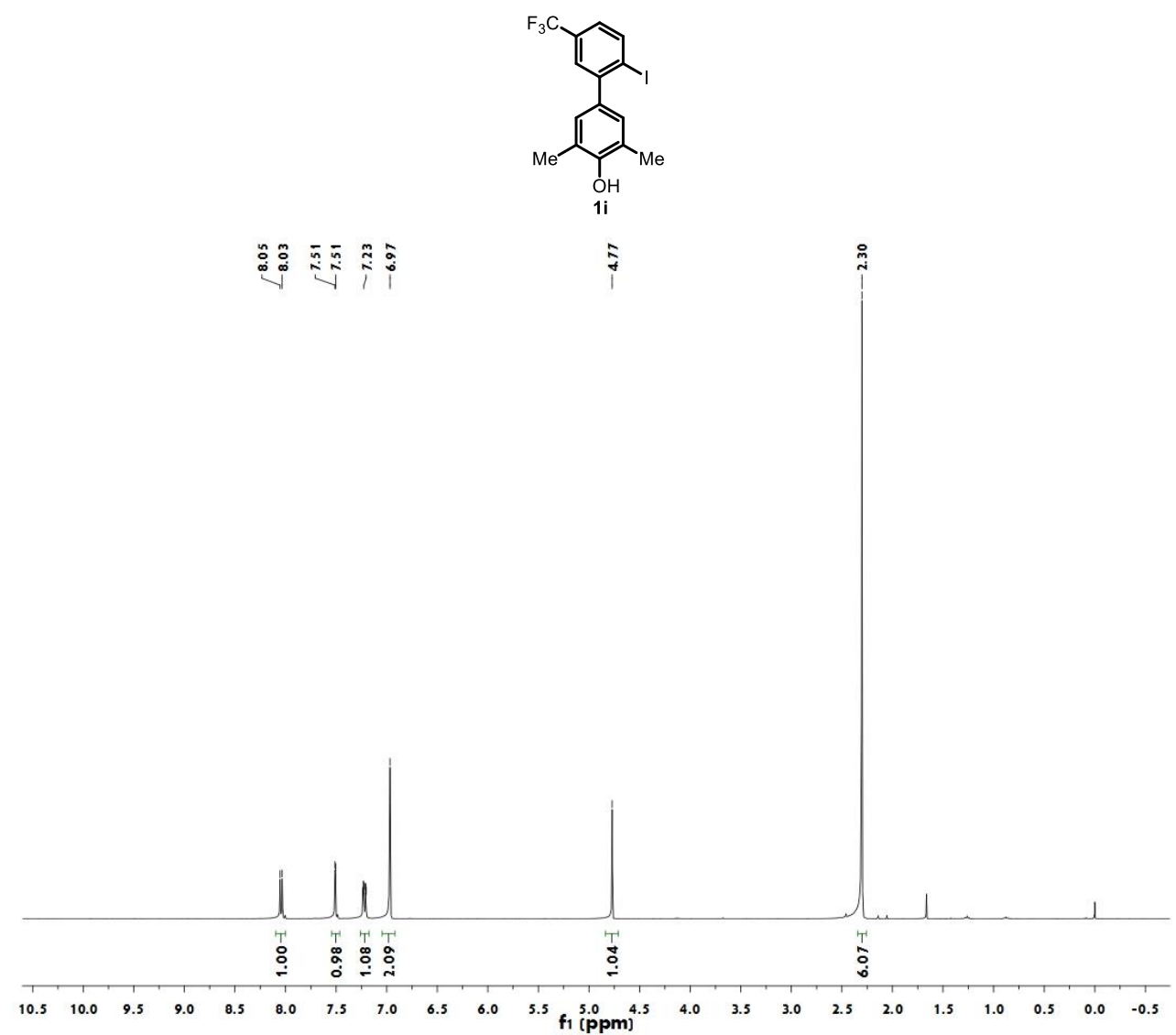

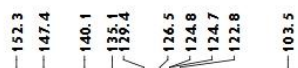

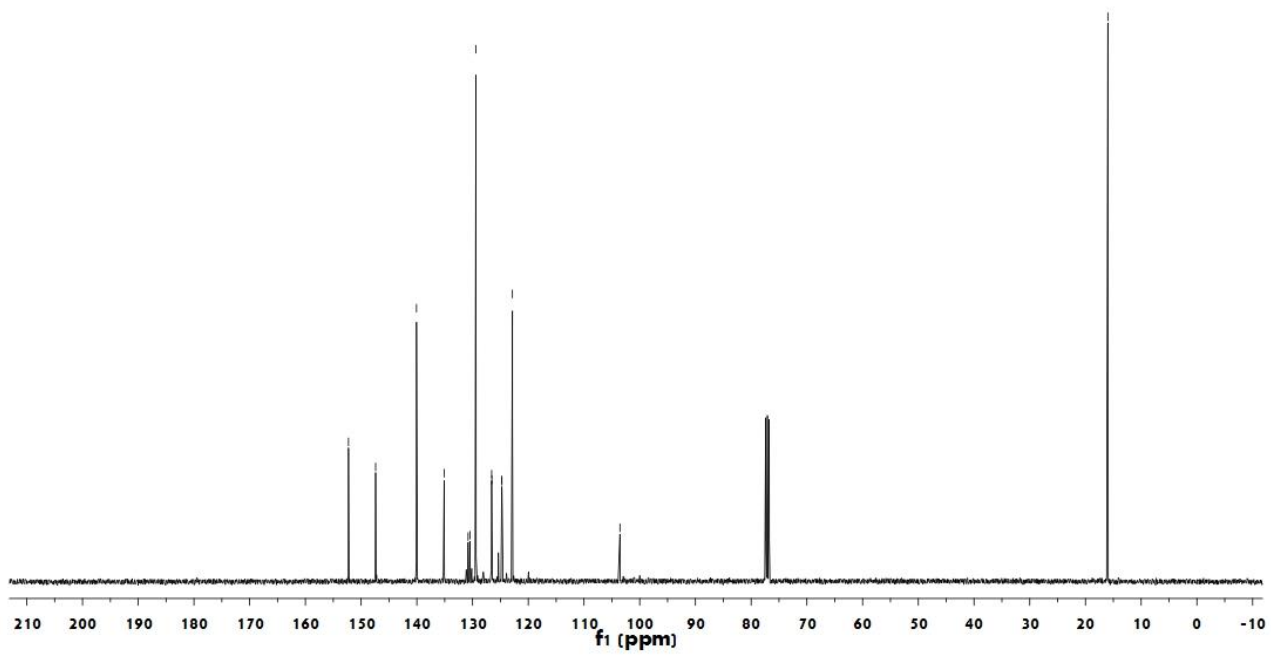




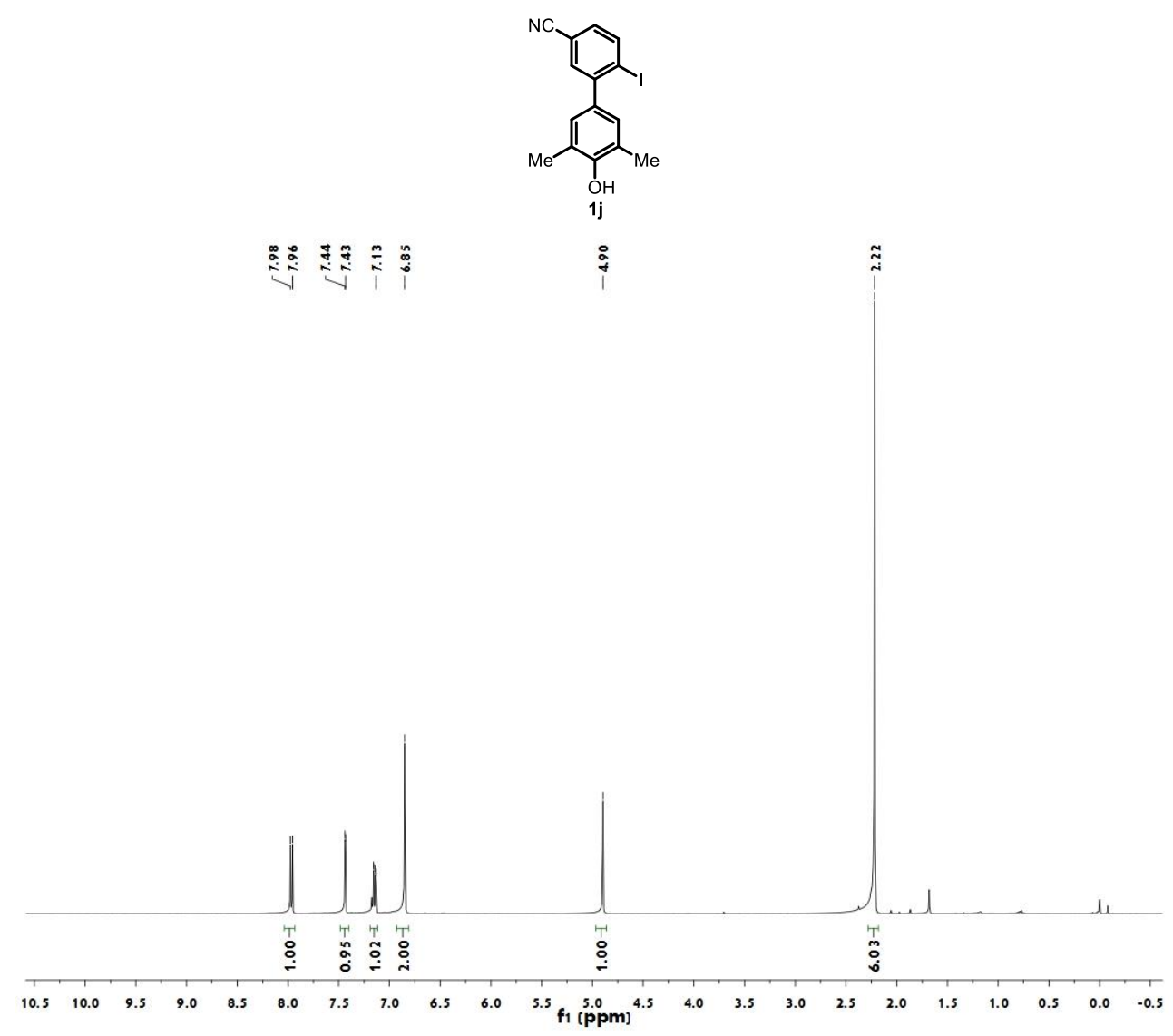

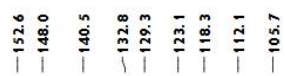

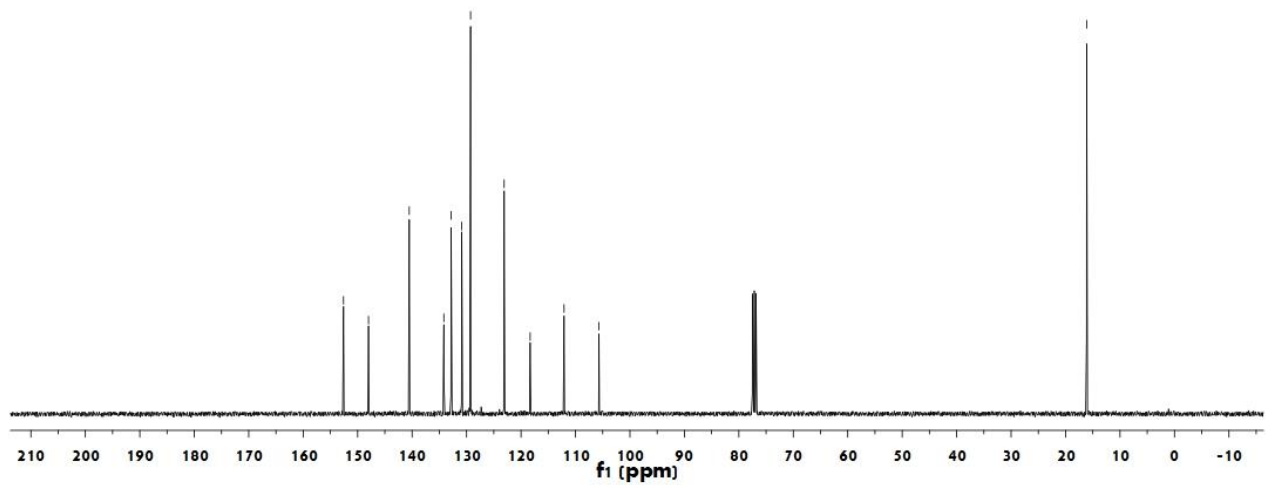




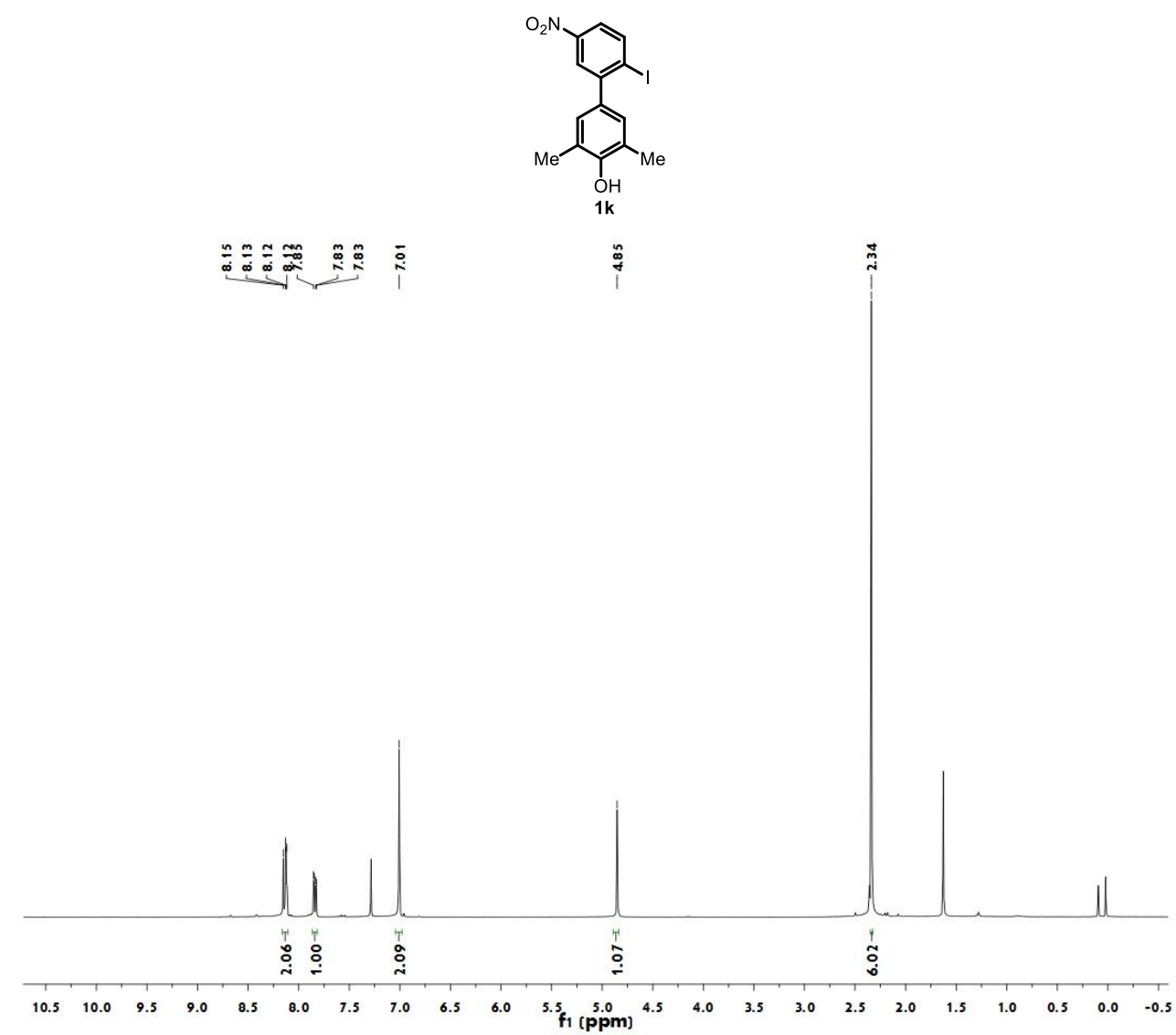

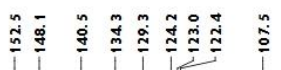

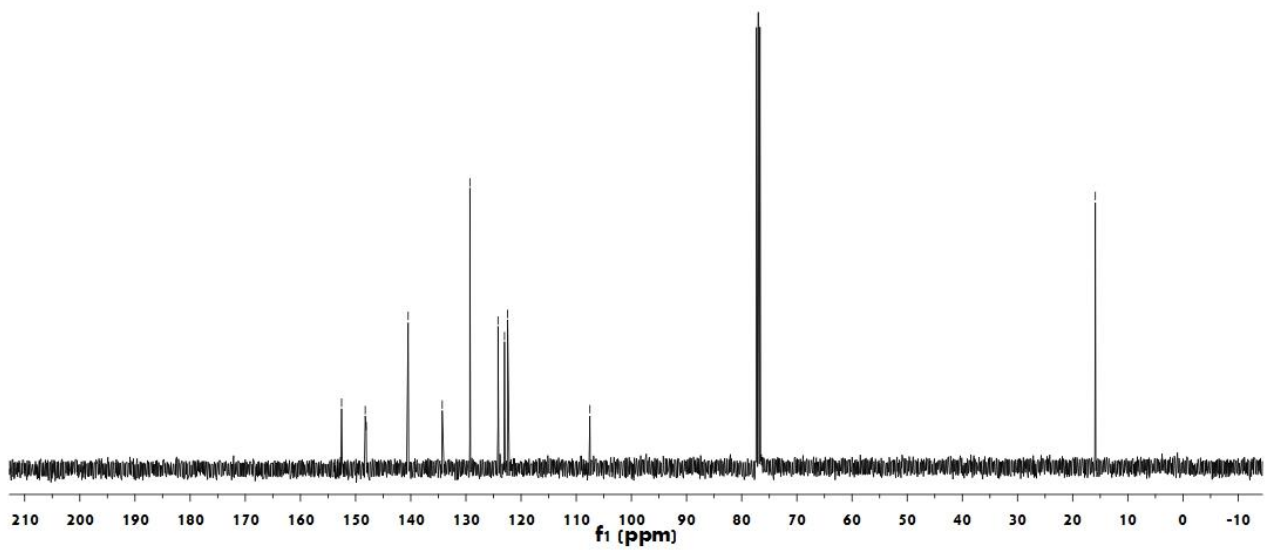



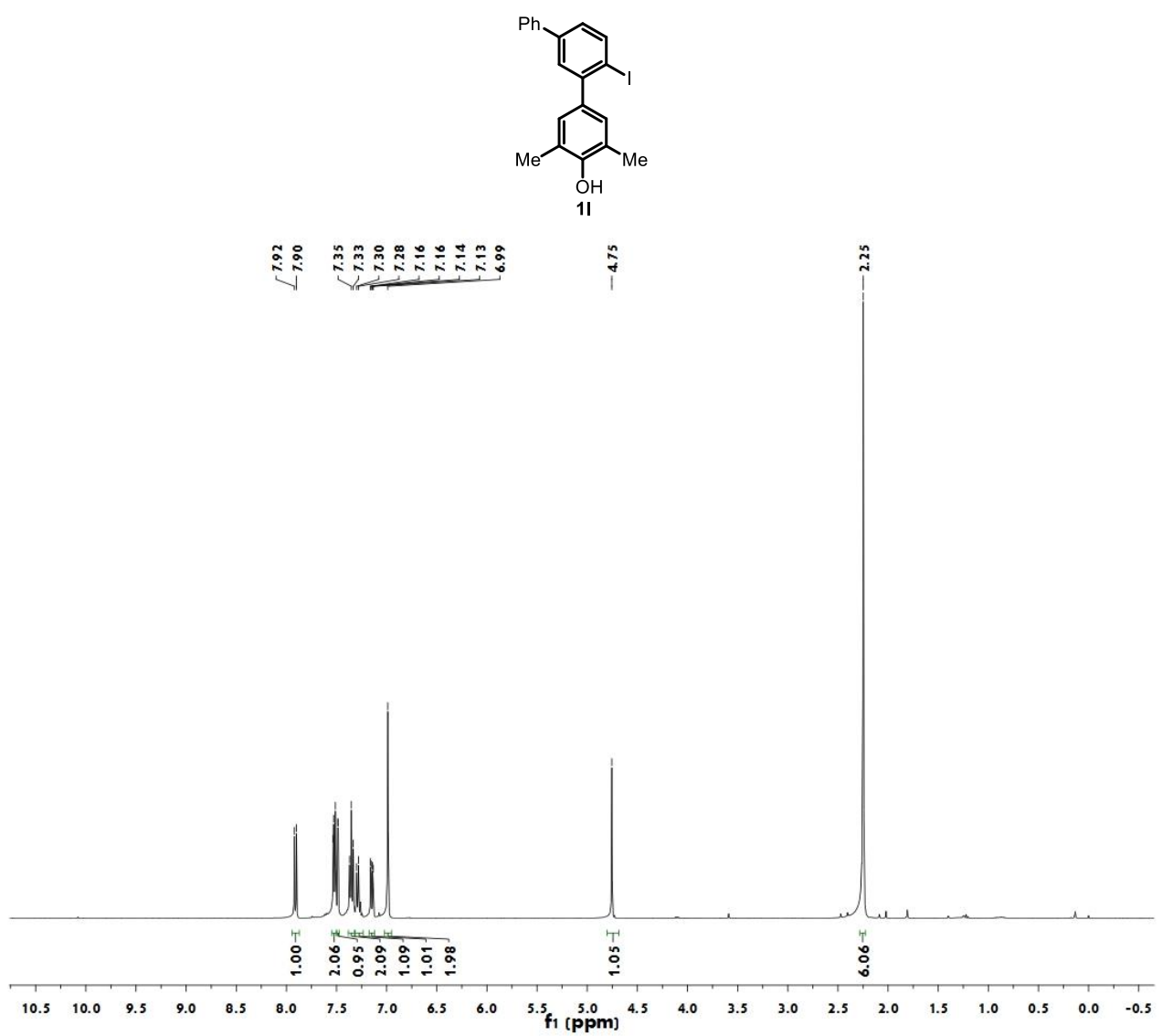

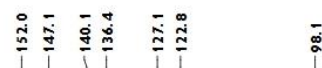

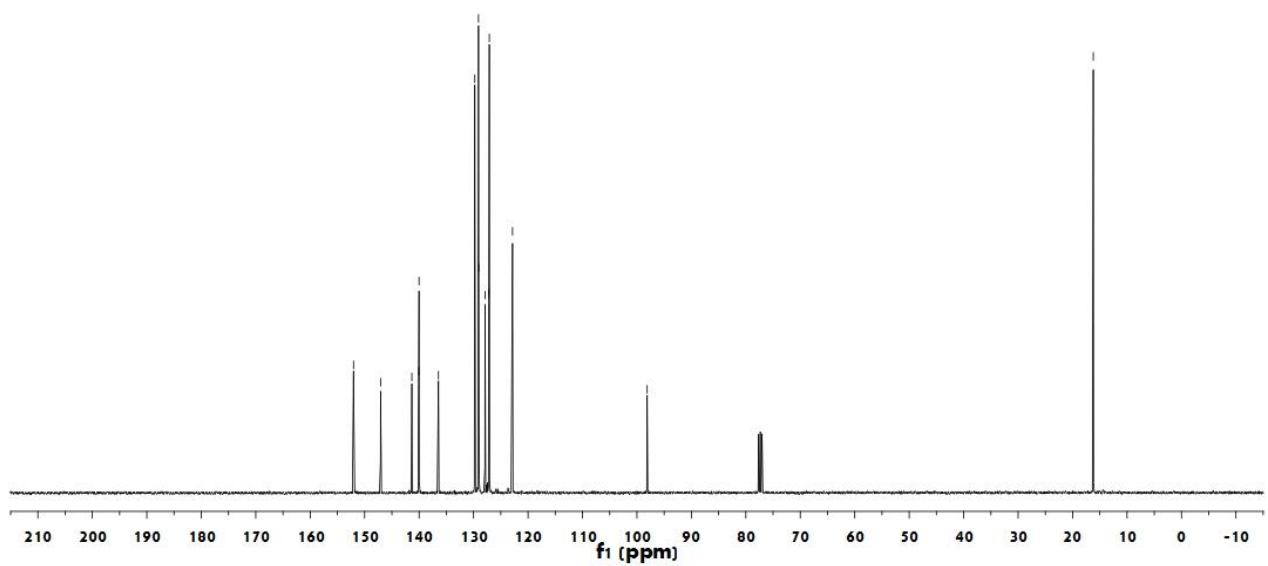



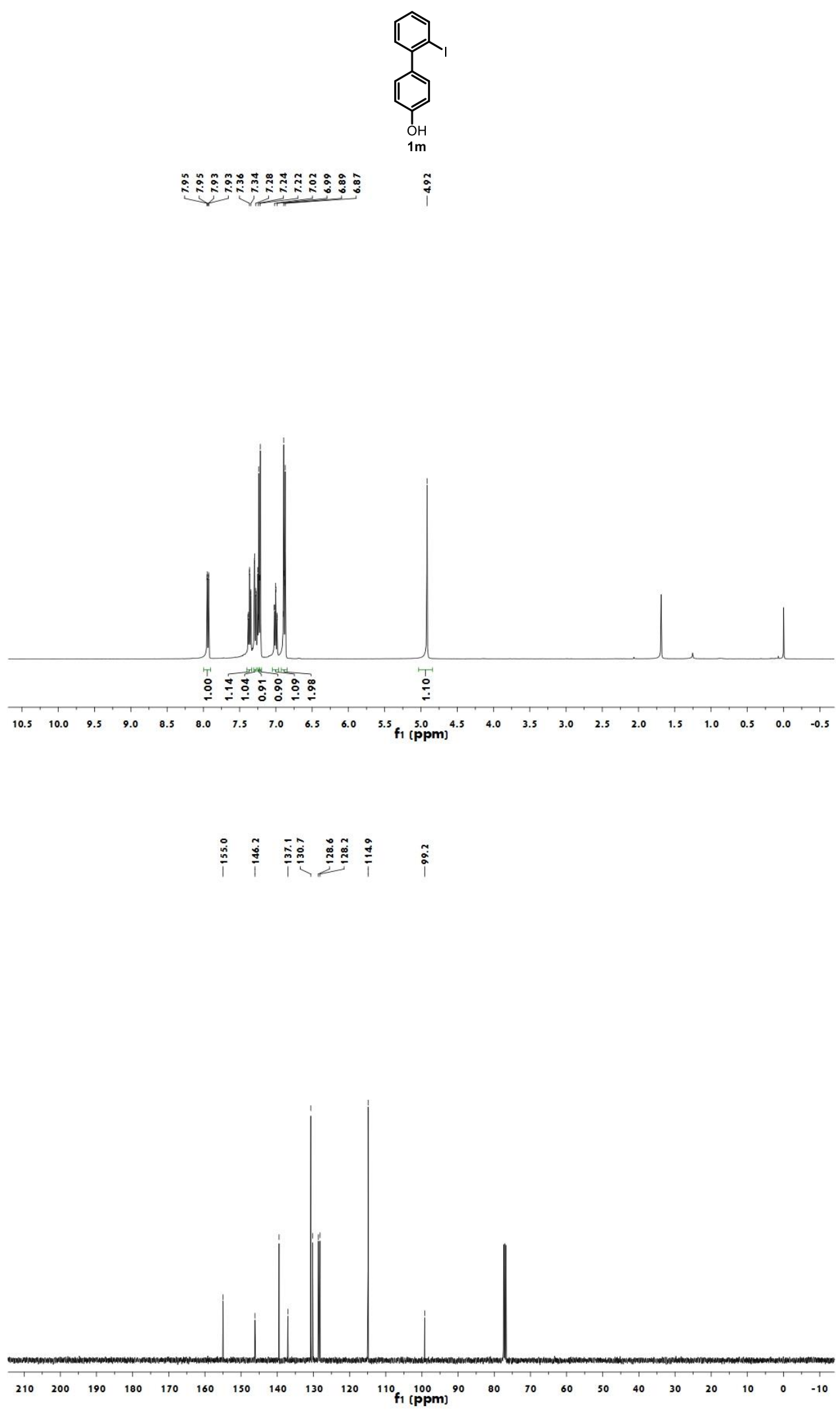


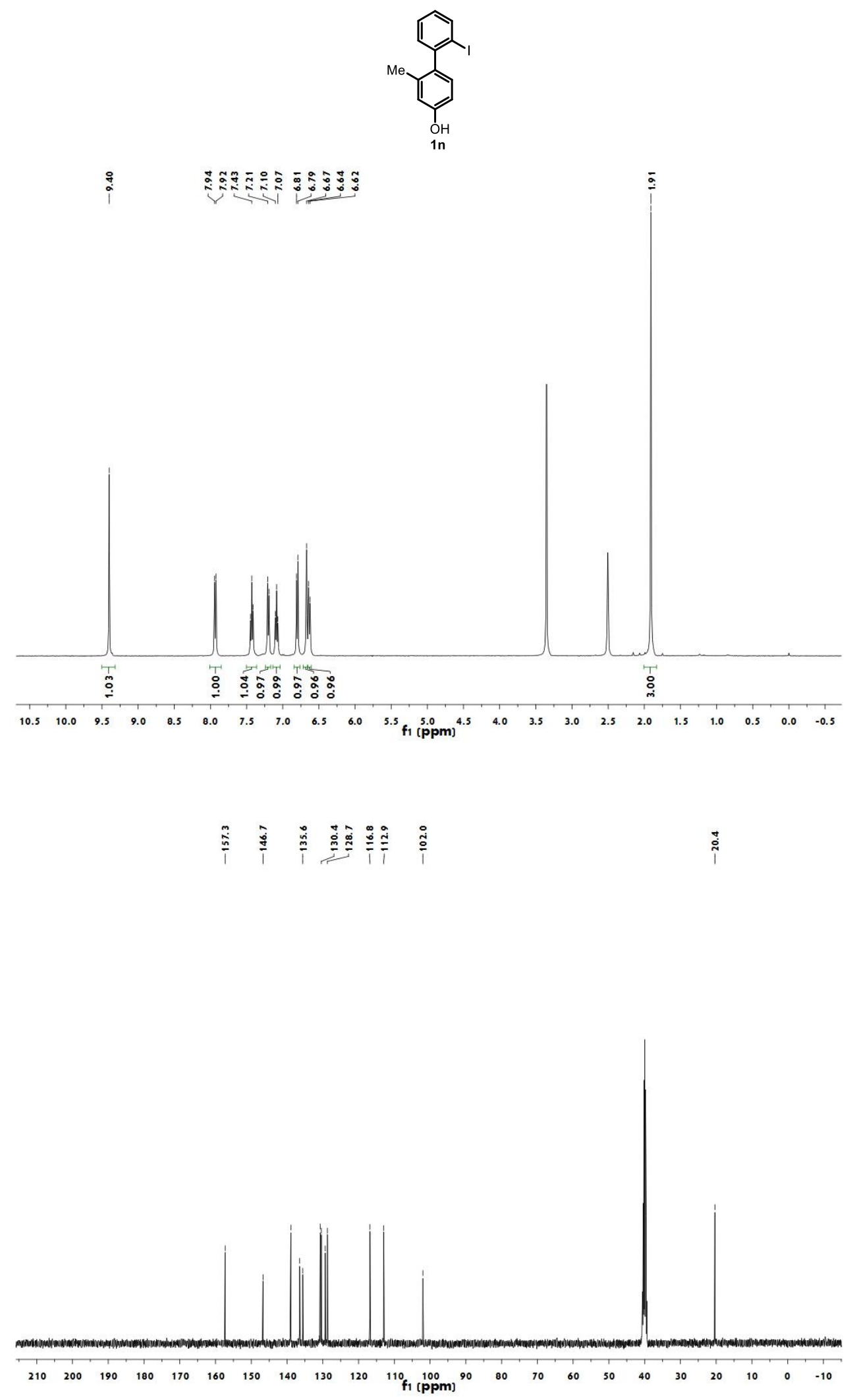



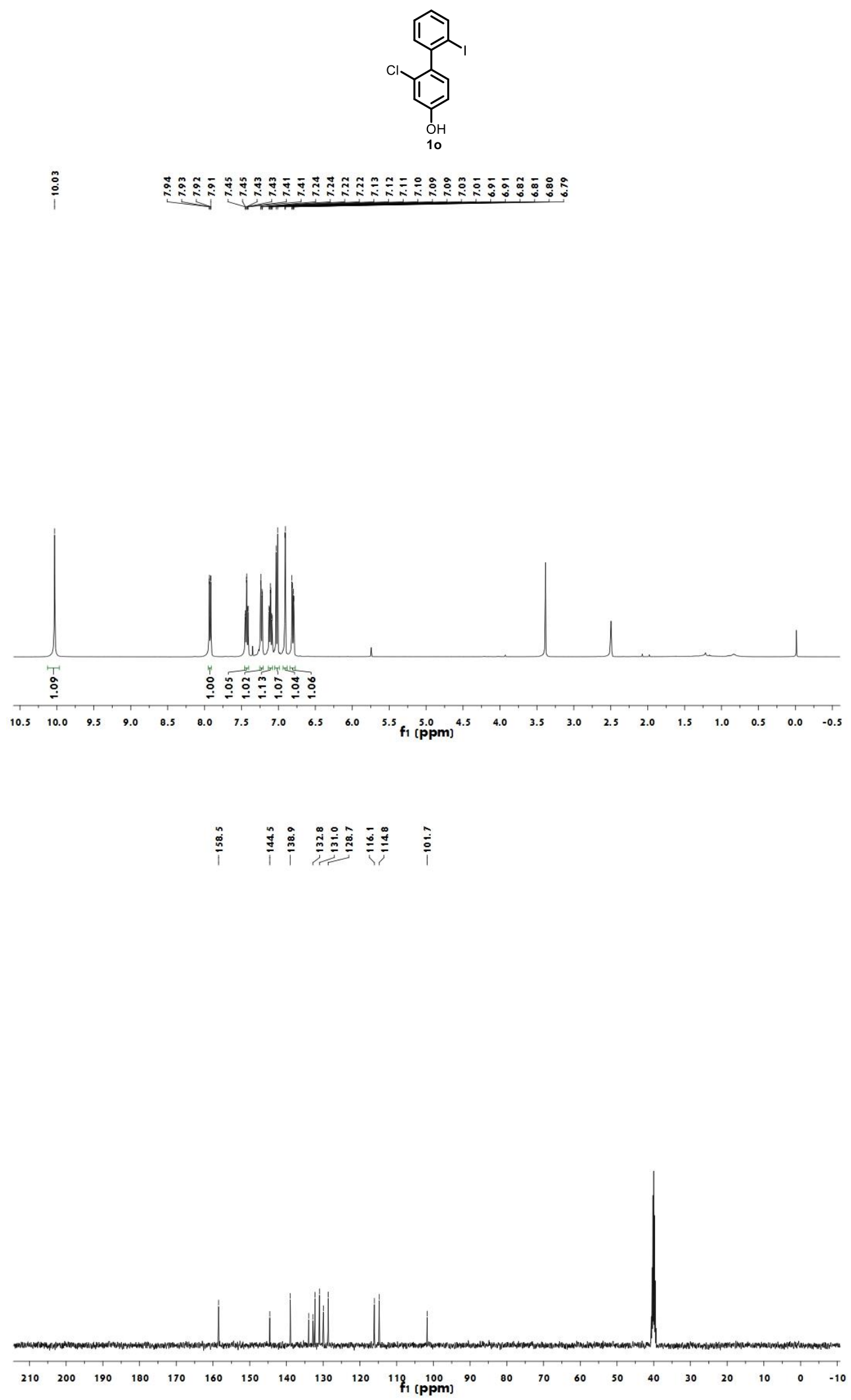

S32 


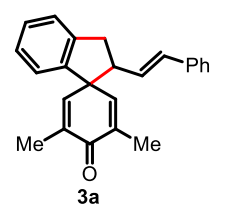

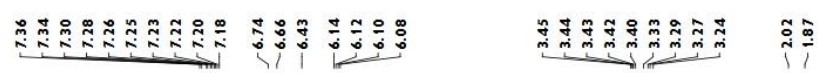

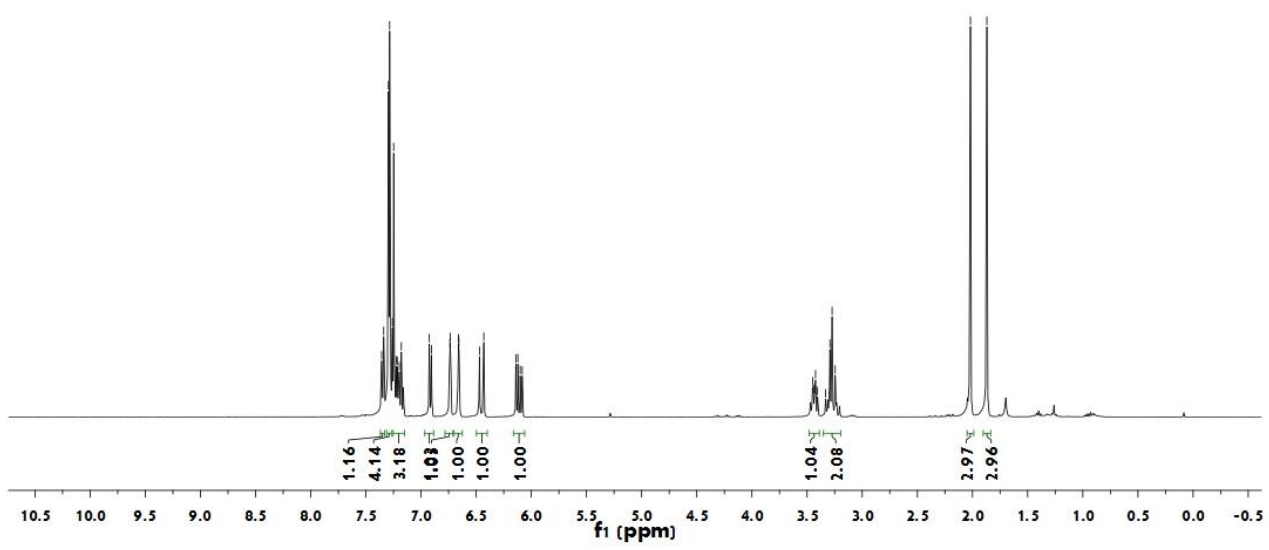

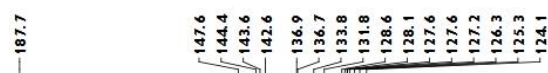

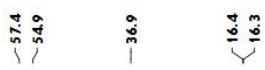

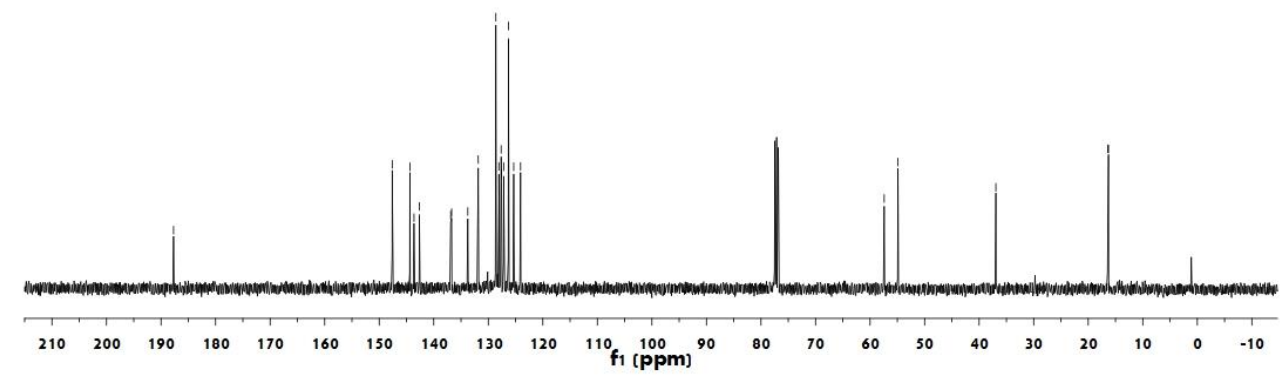




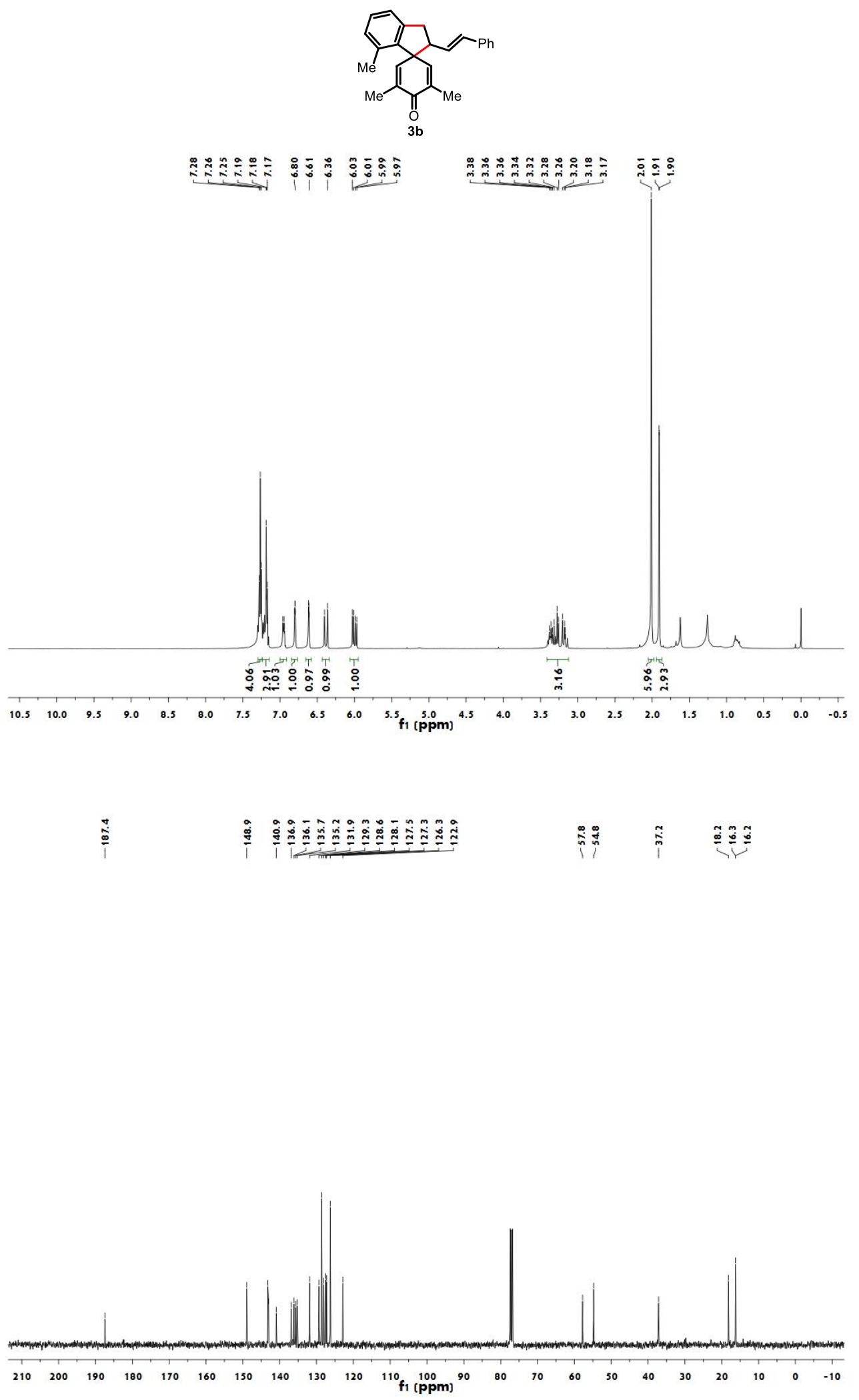




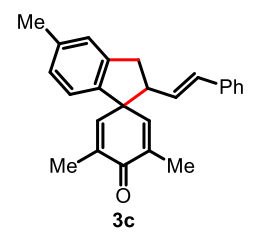

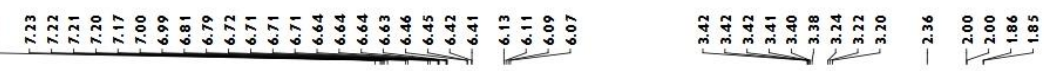

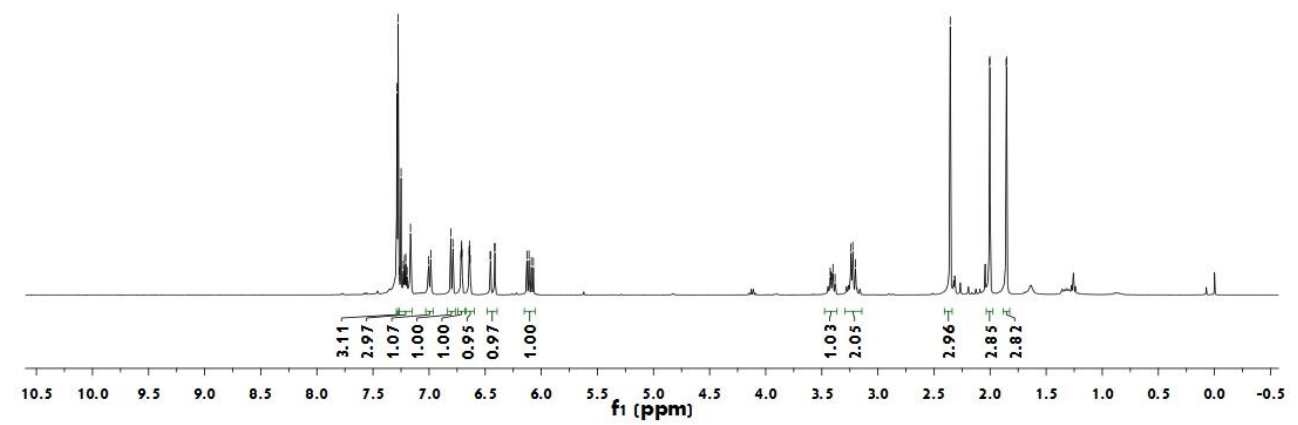

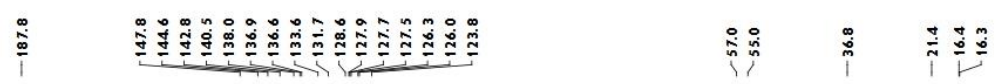

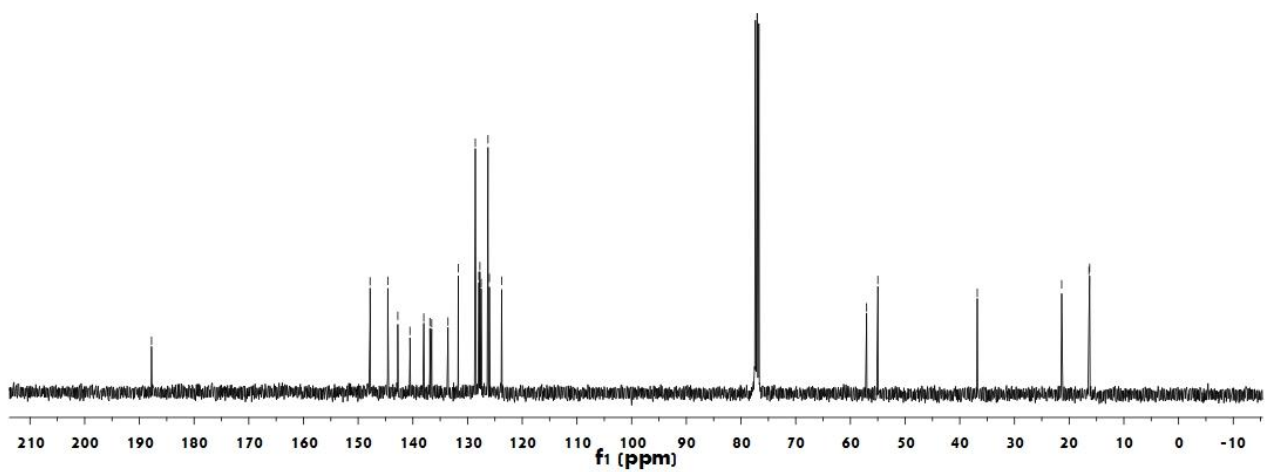




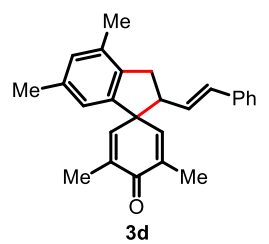

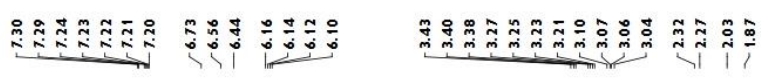
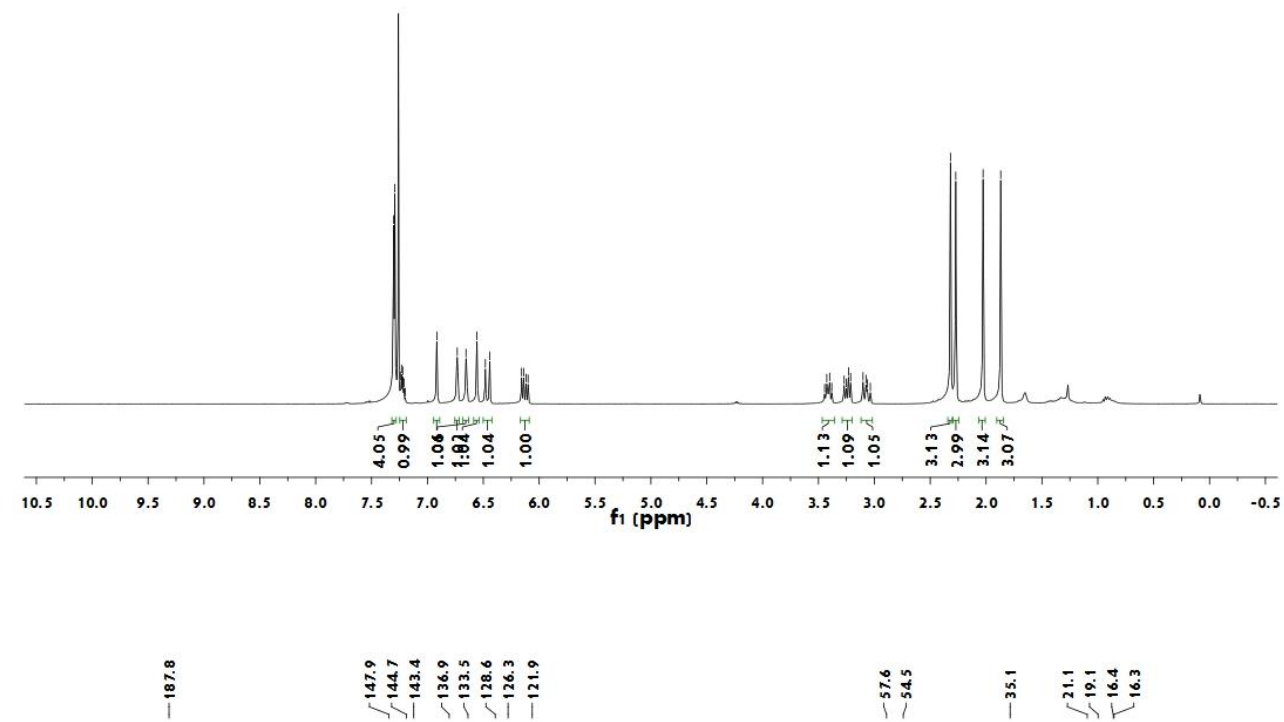

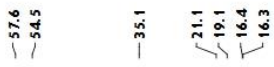

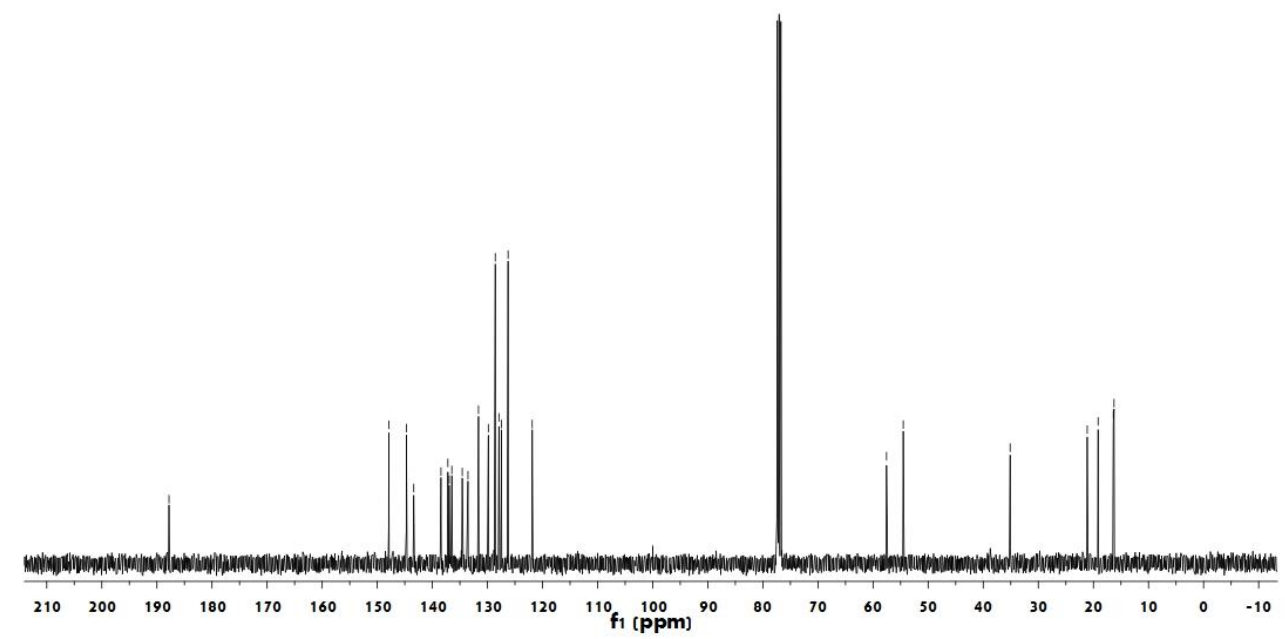




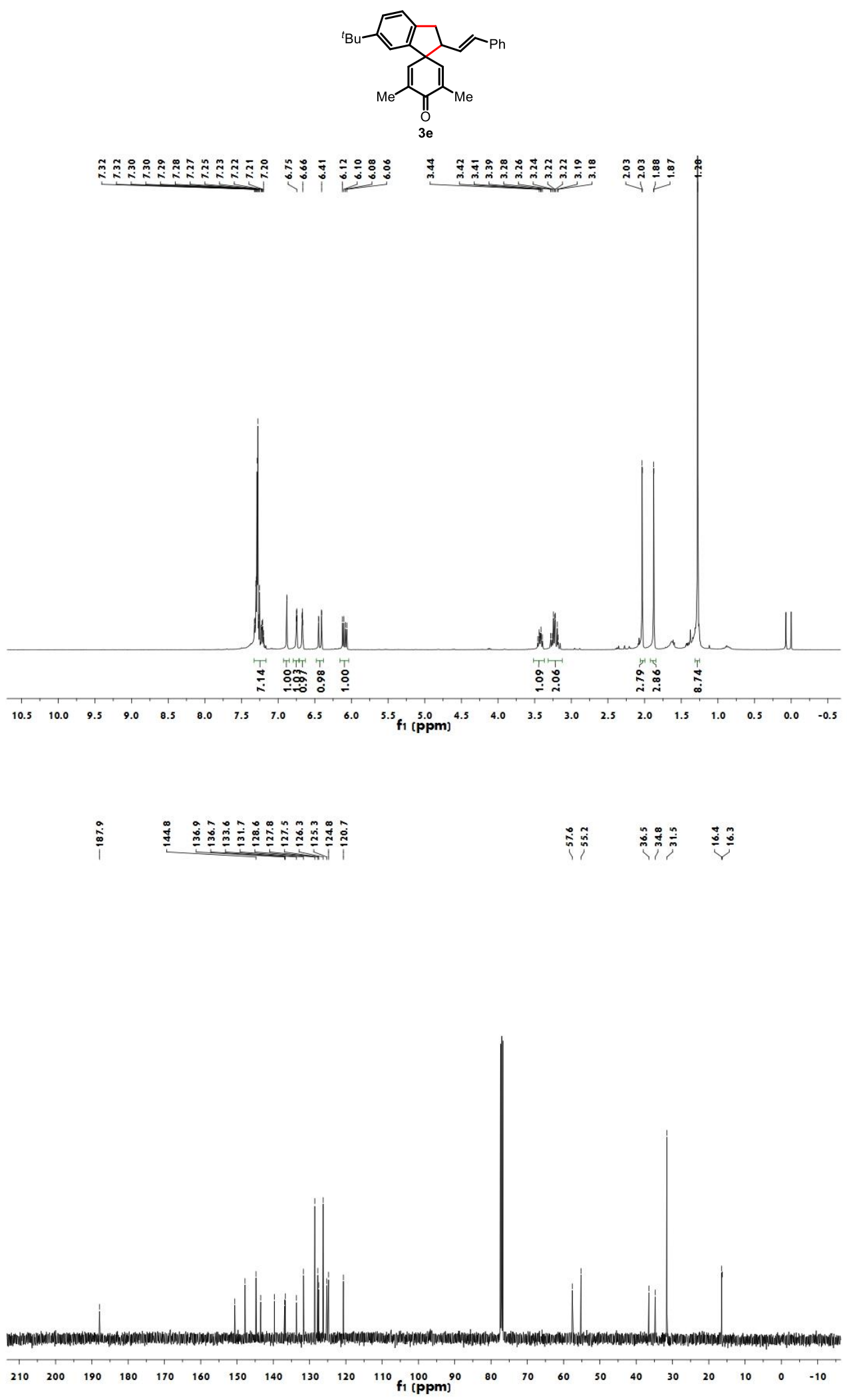



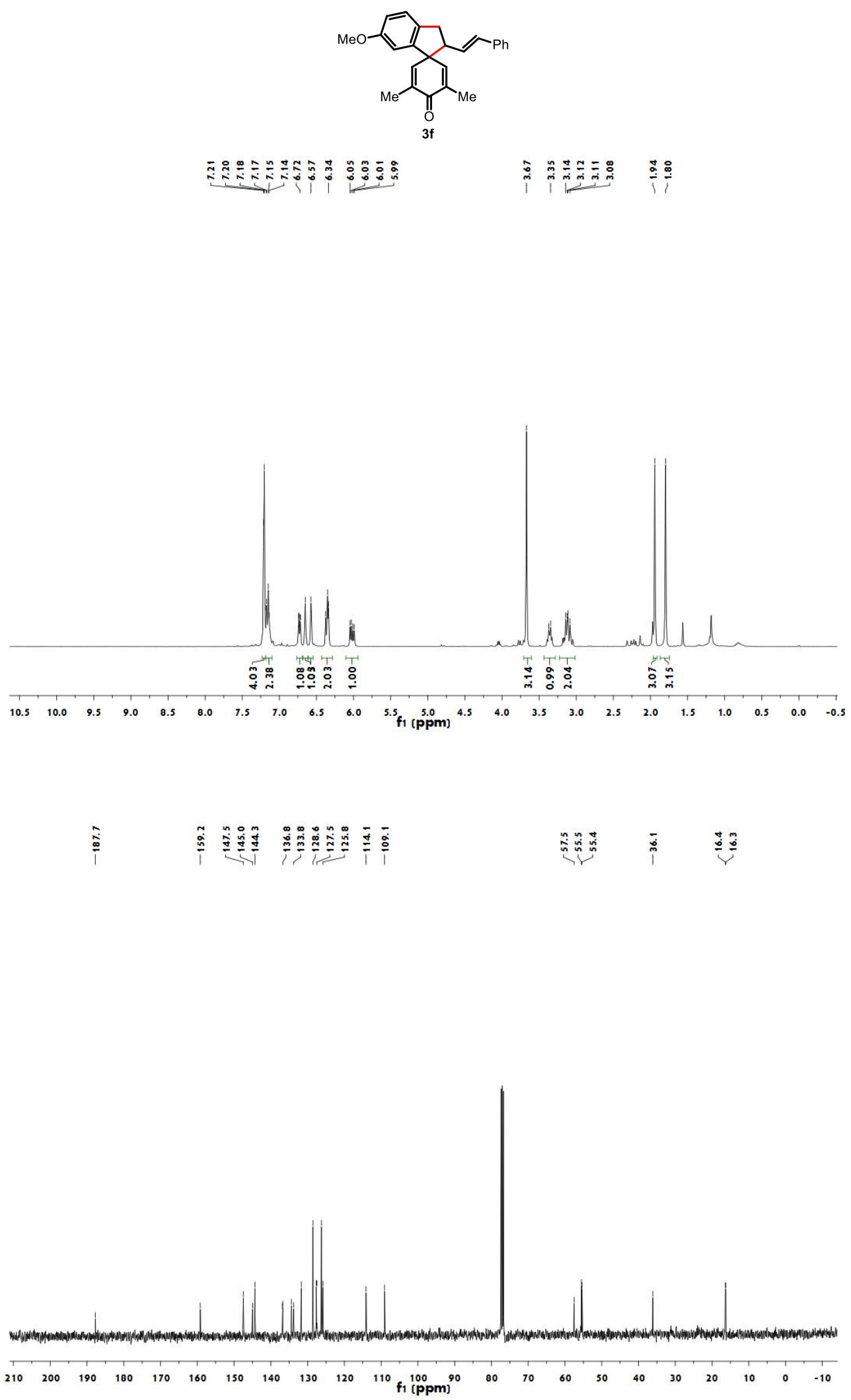


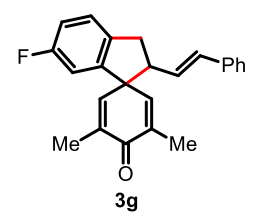

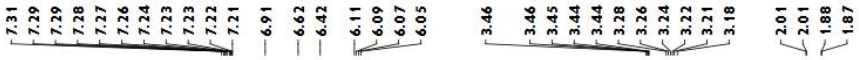
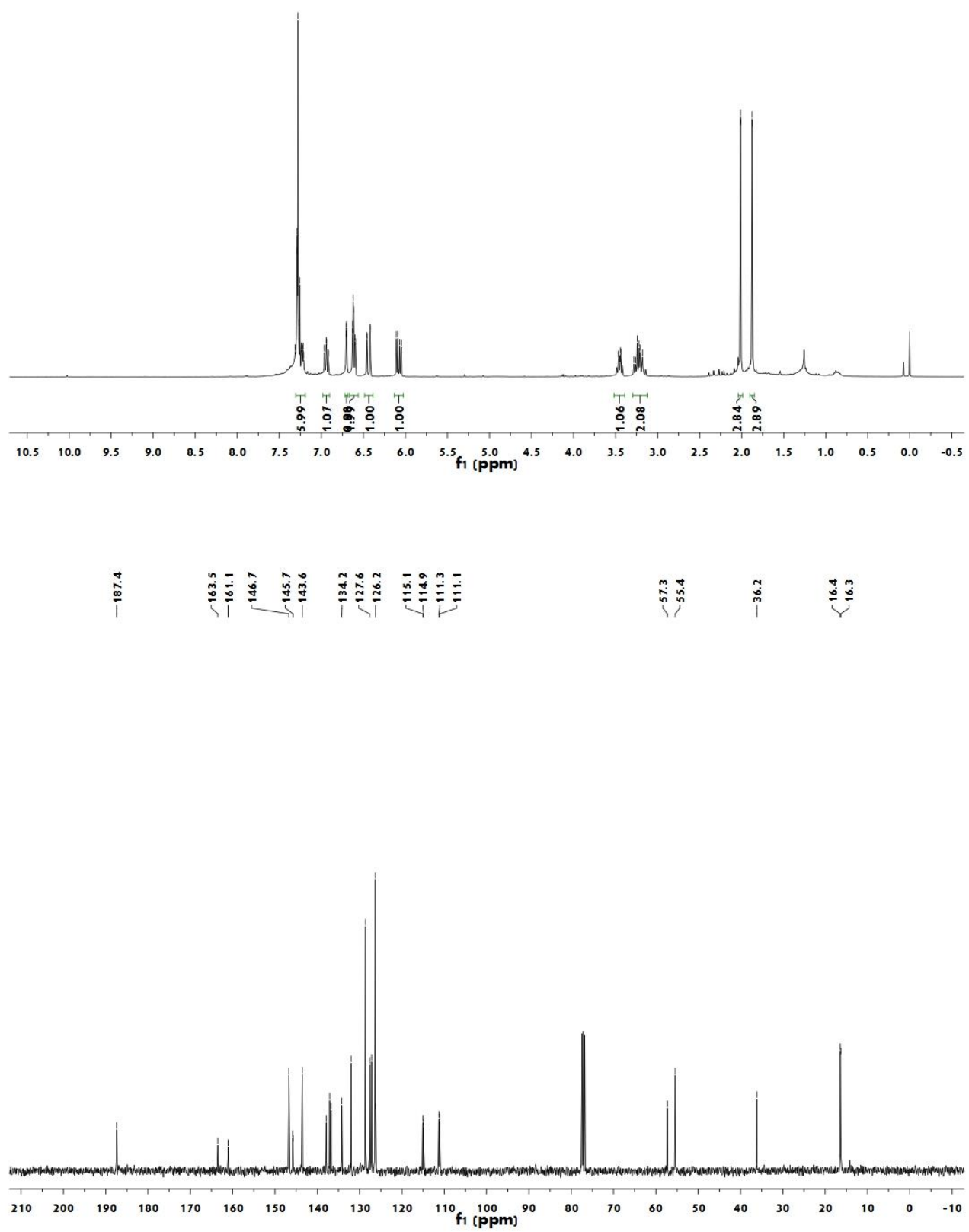

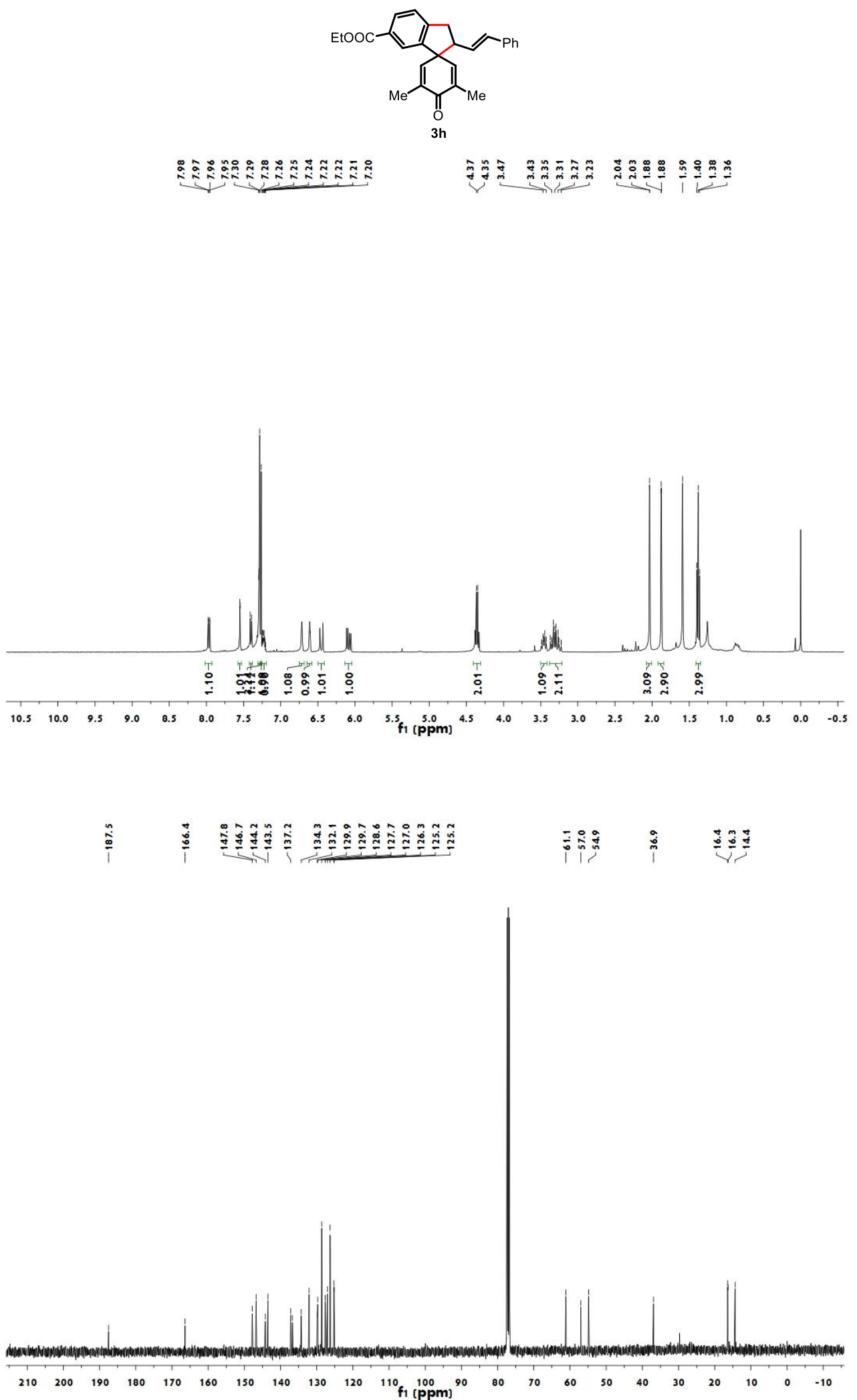


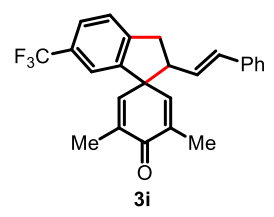

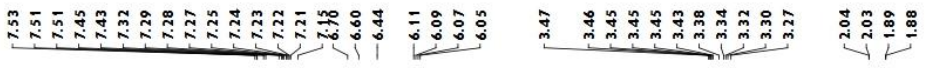
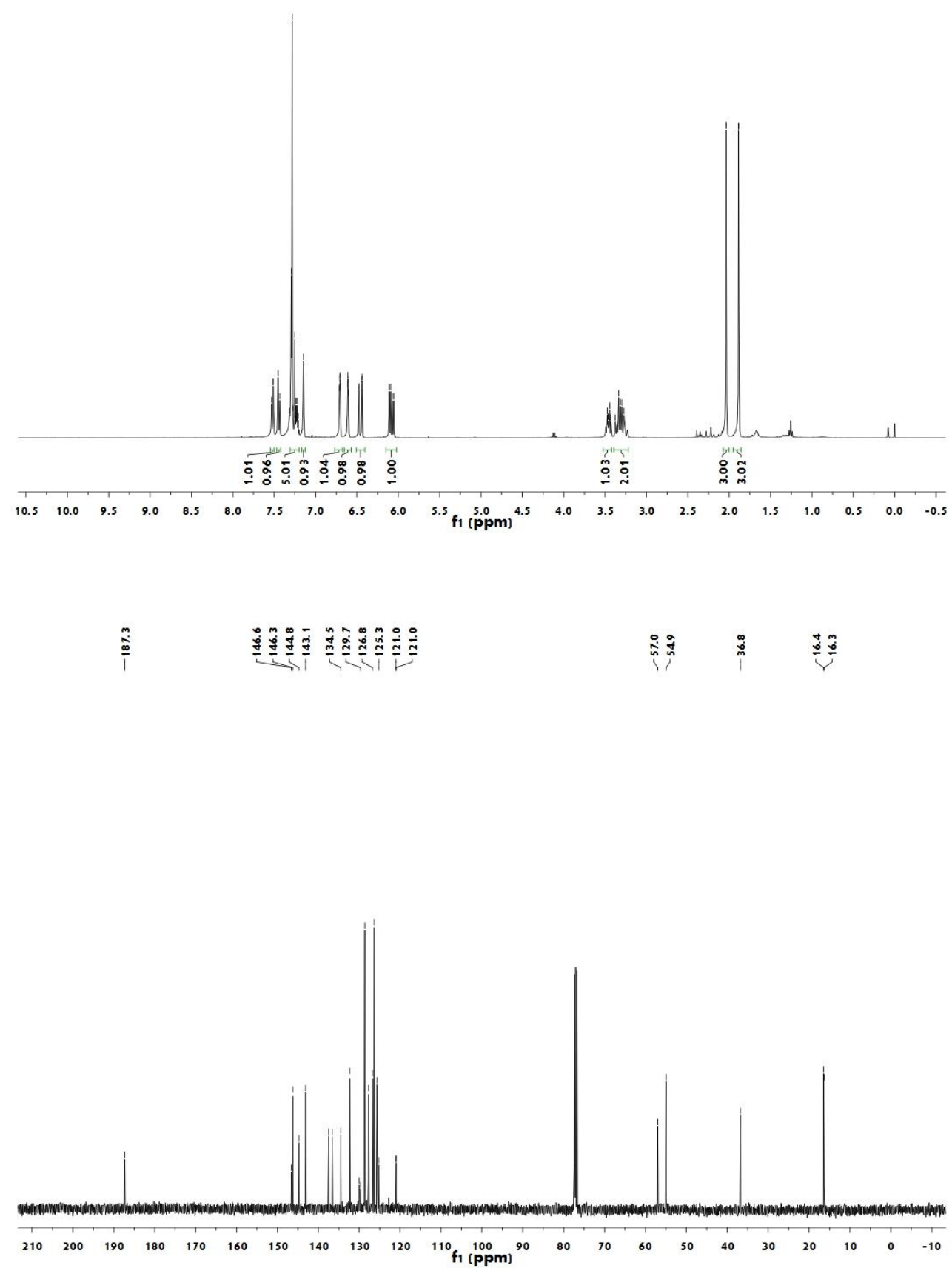


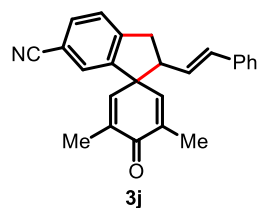

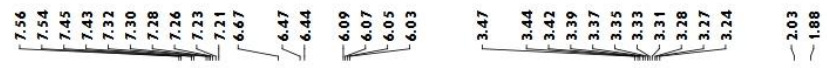
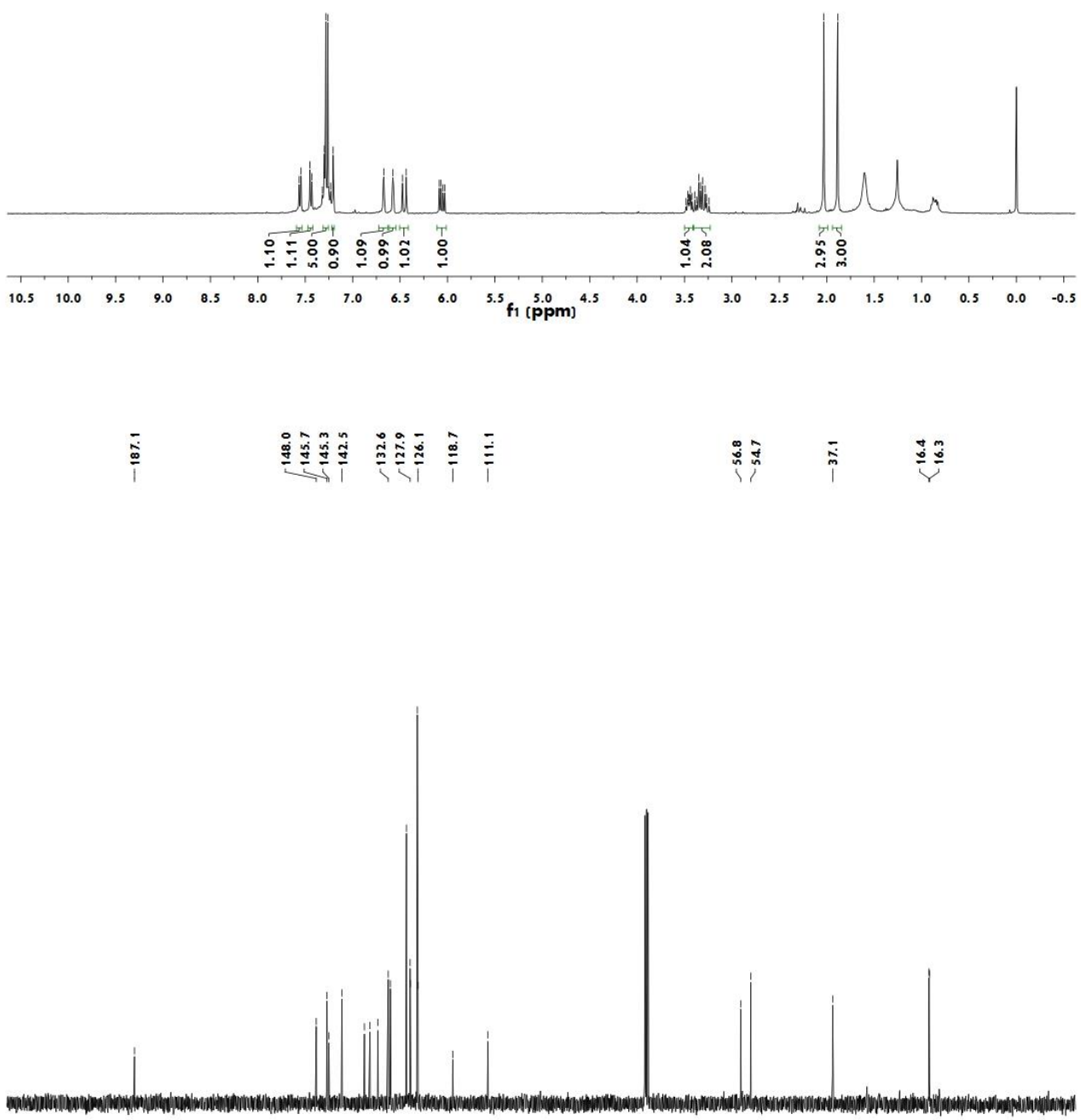

$\begin{array}{lllllllllll}210 & 200 & 190 & 180 & 170 & 160 & 150 & 140 & 130 & 120 & { }^{110} \mathrm{f}_{1}\left(\begin{array}{l}100 \\ \text { (p pm })\end{array}\right.\end{array}$ 

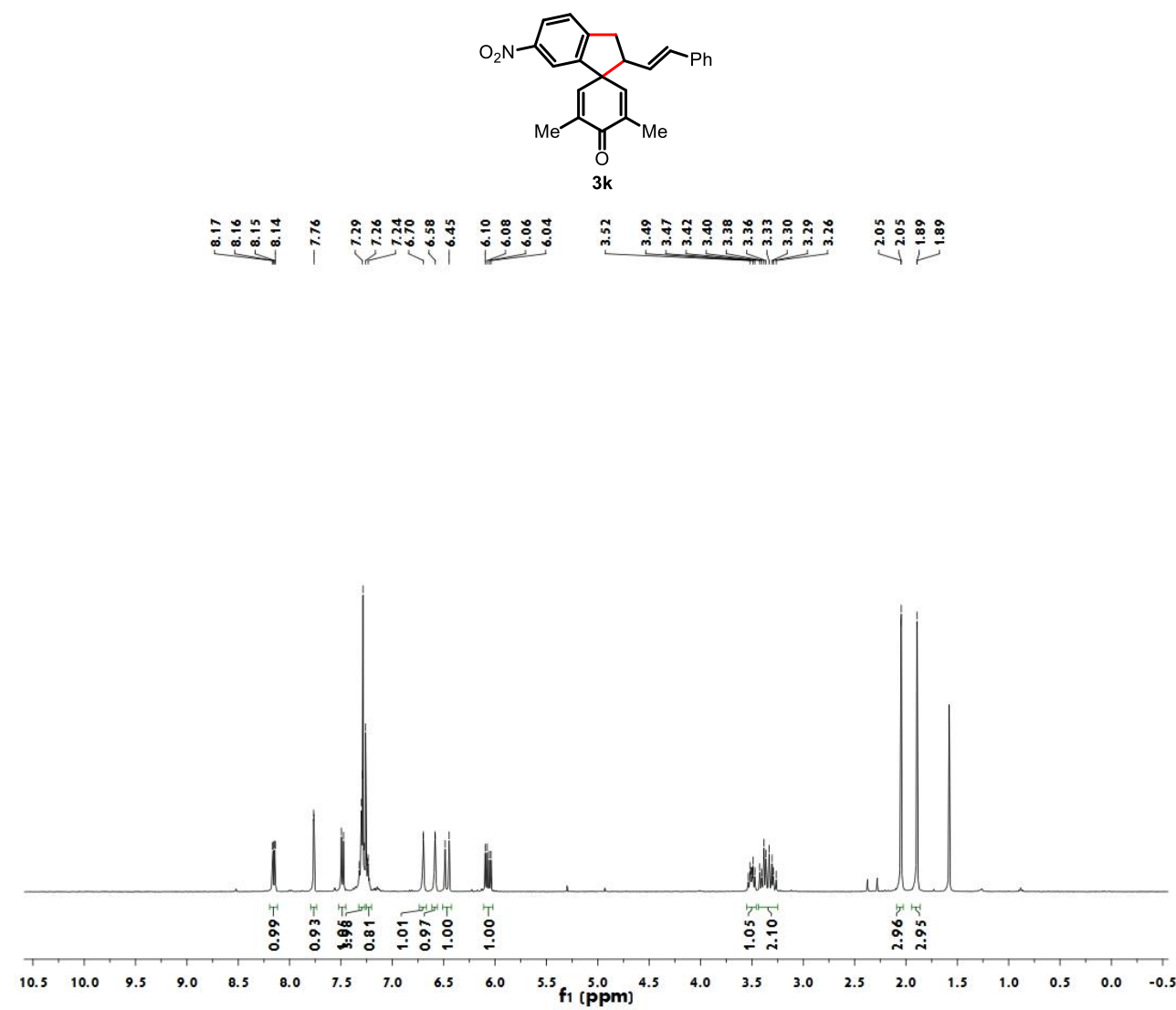

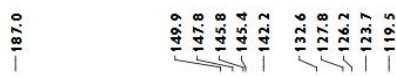

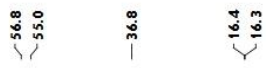

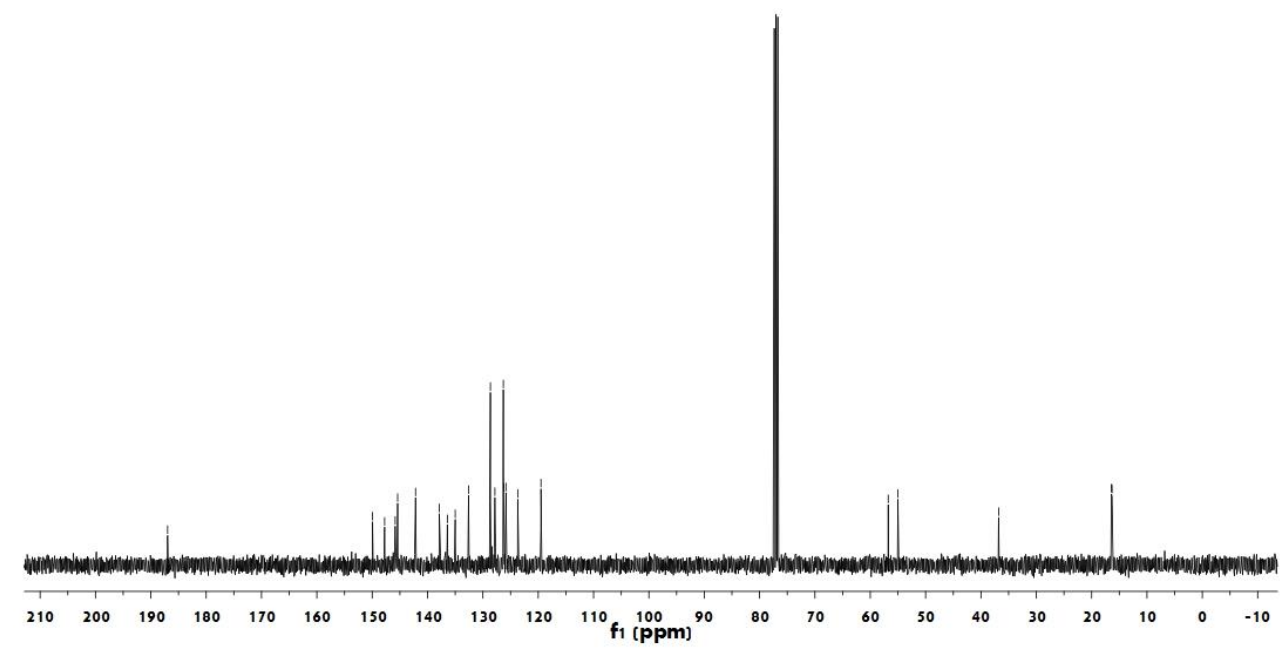




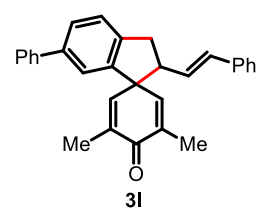

\section{象}
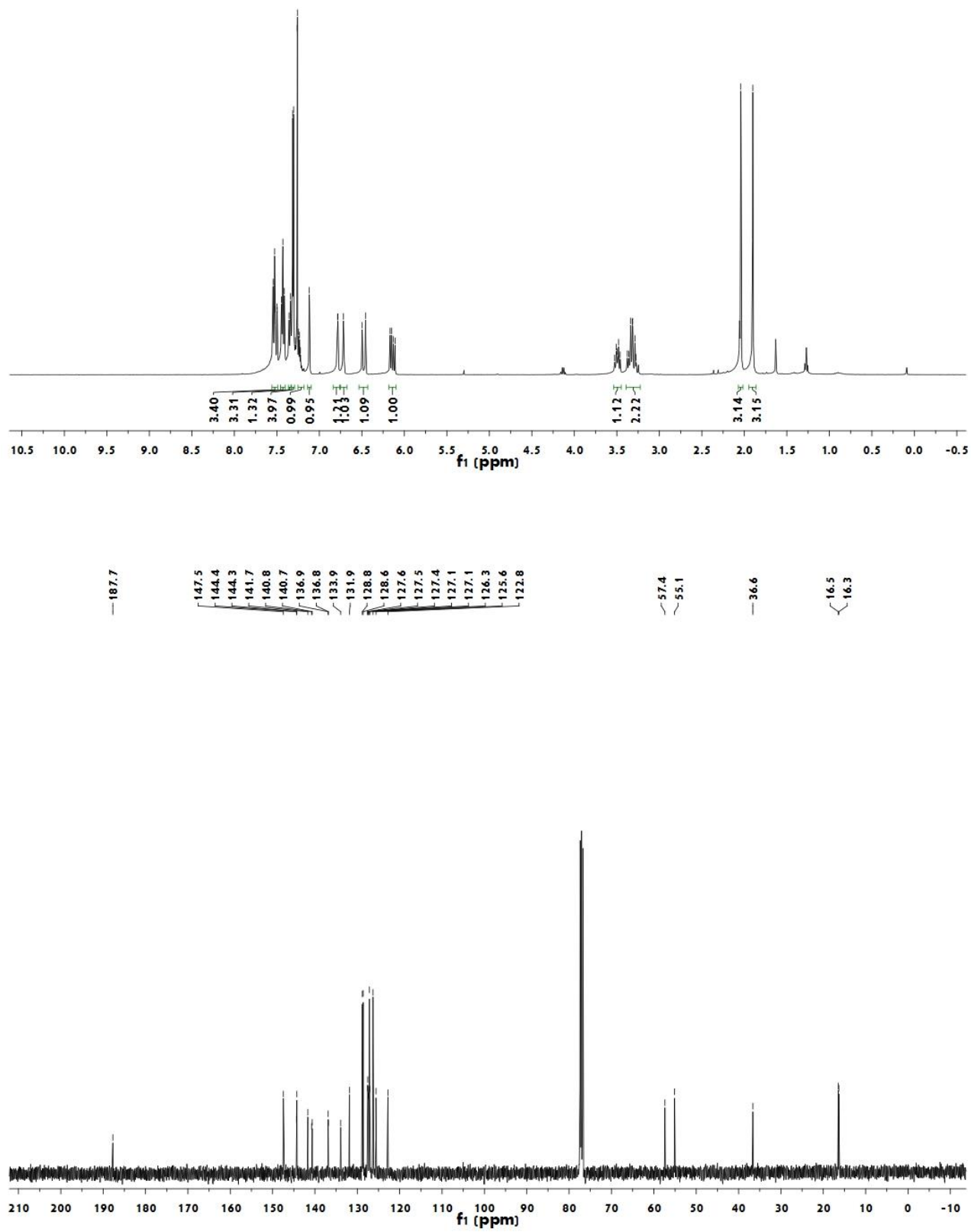


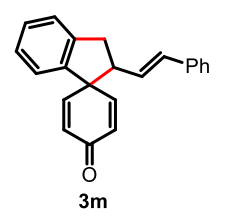

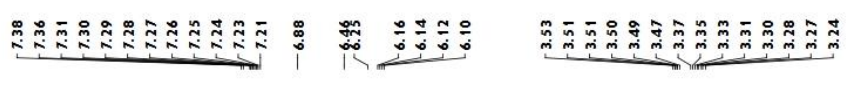
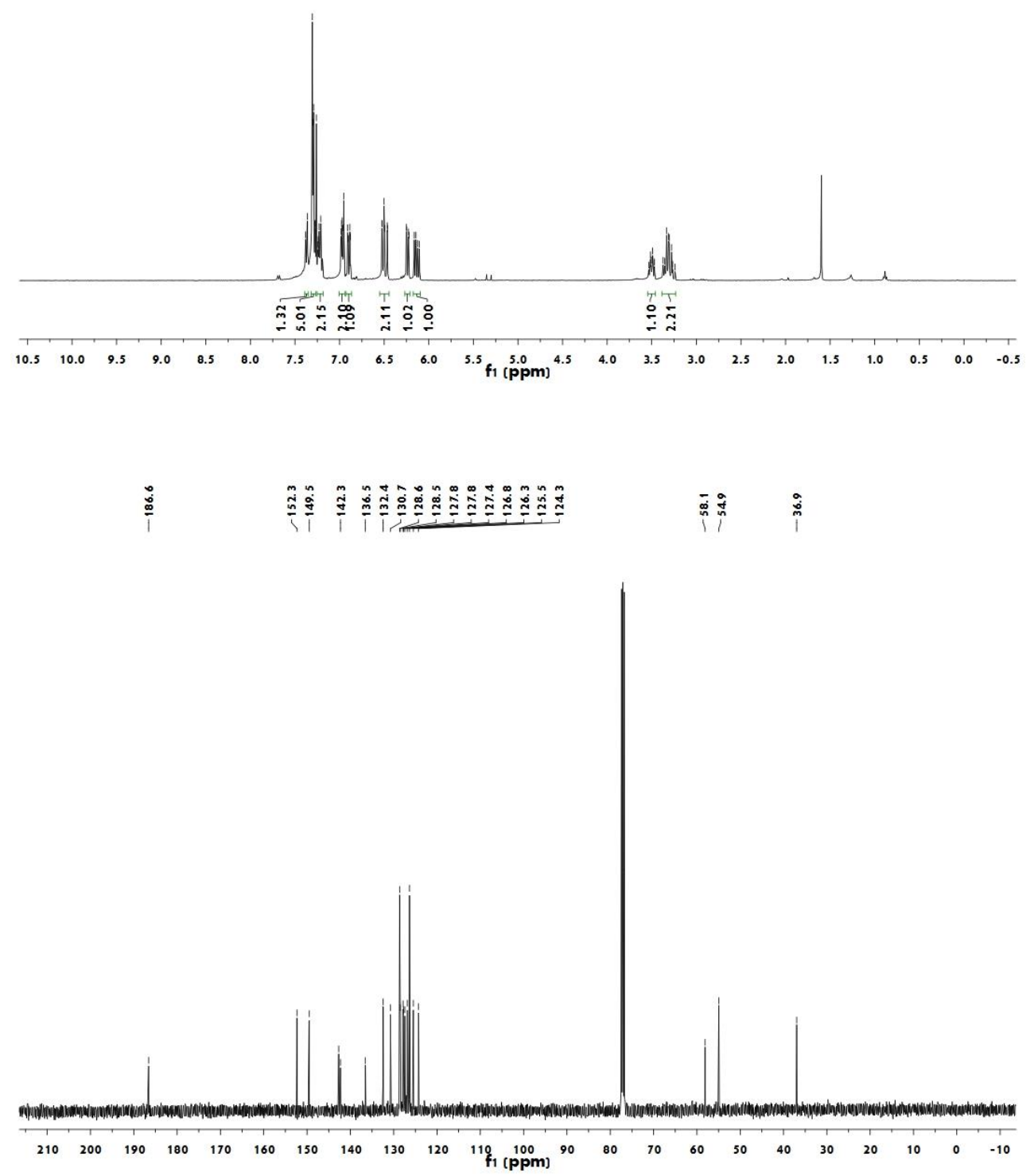


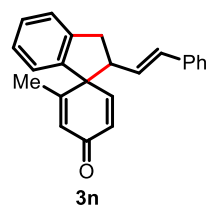

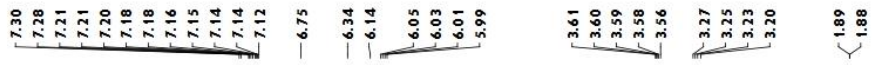

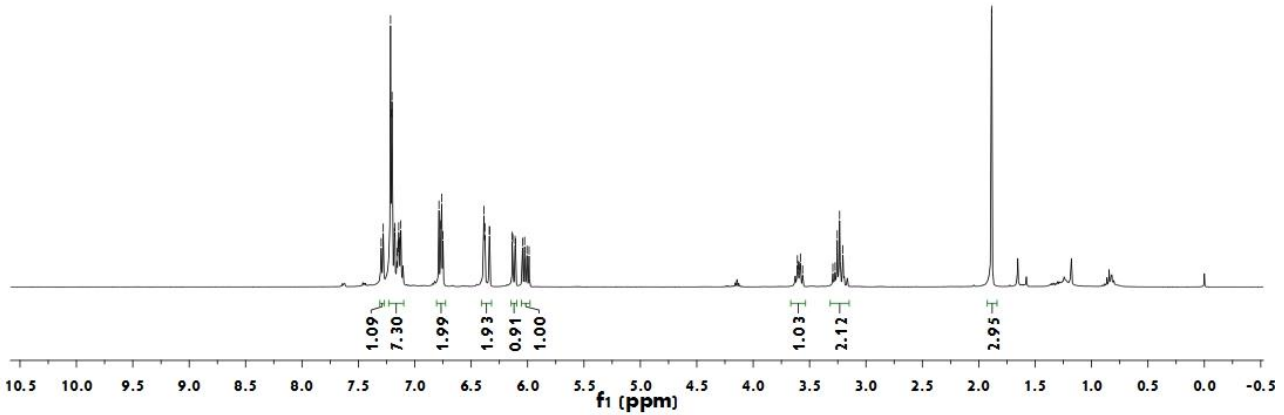

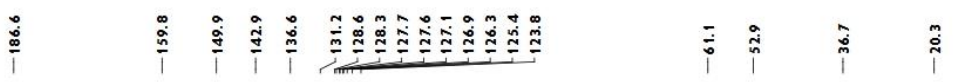

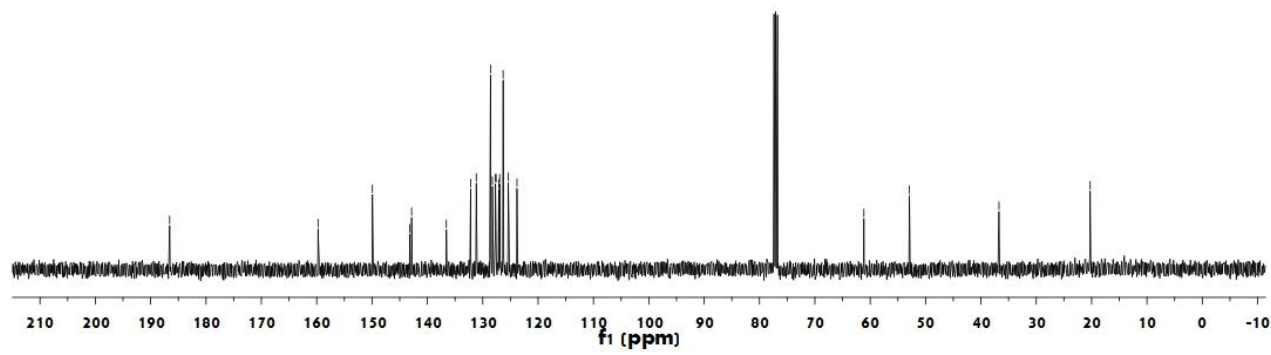




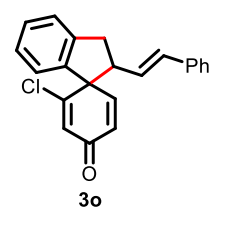

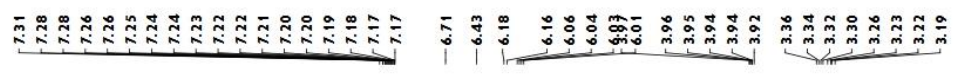

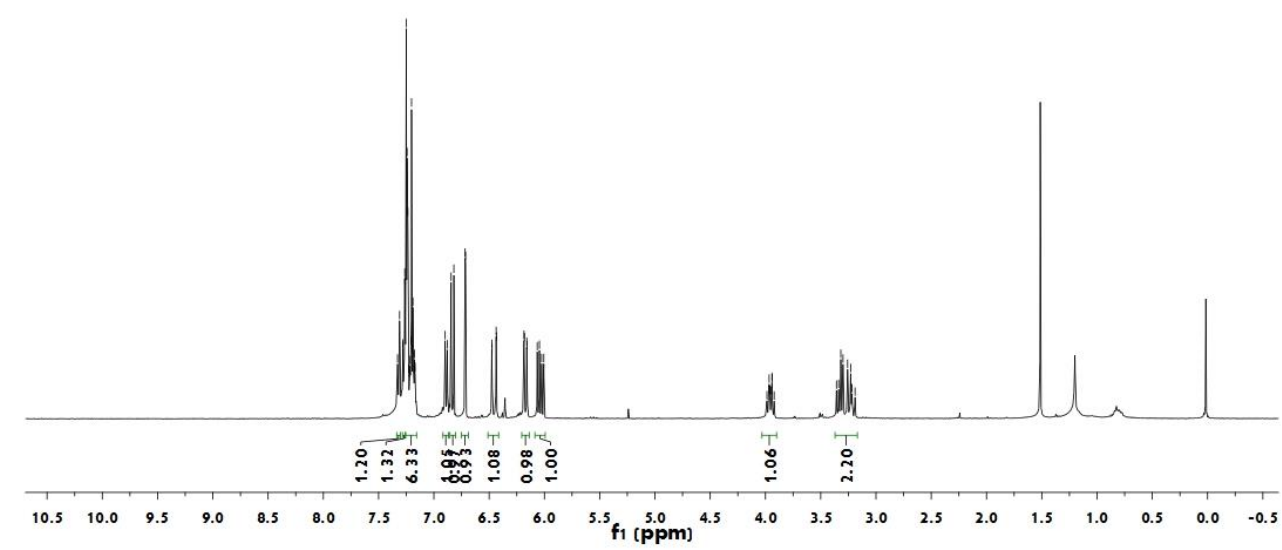

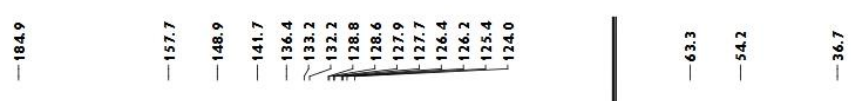

$\begin{array}{lllllllllll}210 & 200 & 190 & 180 & 170 & 160 & 150 & 140 & 130 & 120 & 110 \\ f_{1}(\mathrm{ppm}) & 100\end{array}$ 


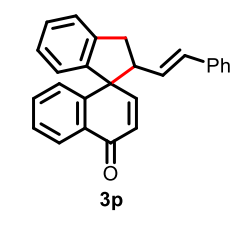

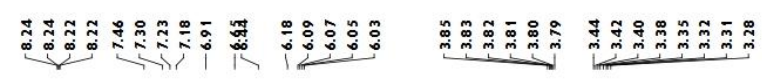
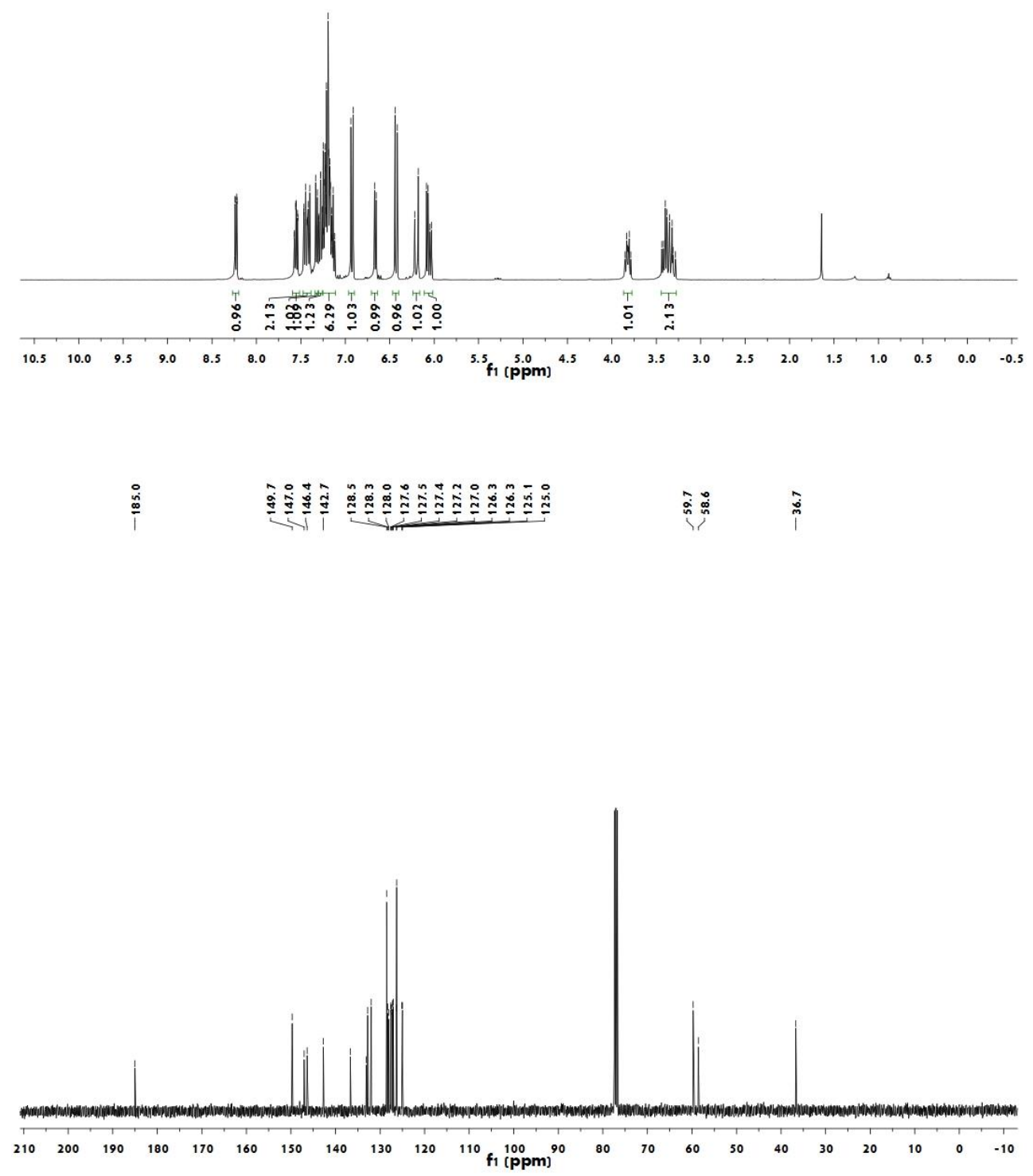


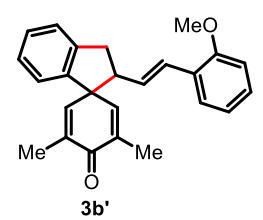

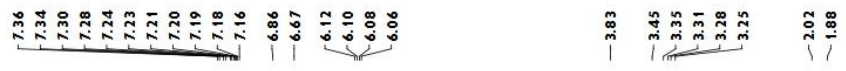

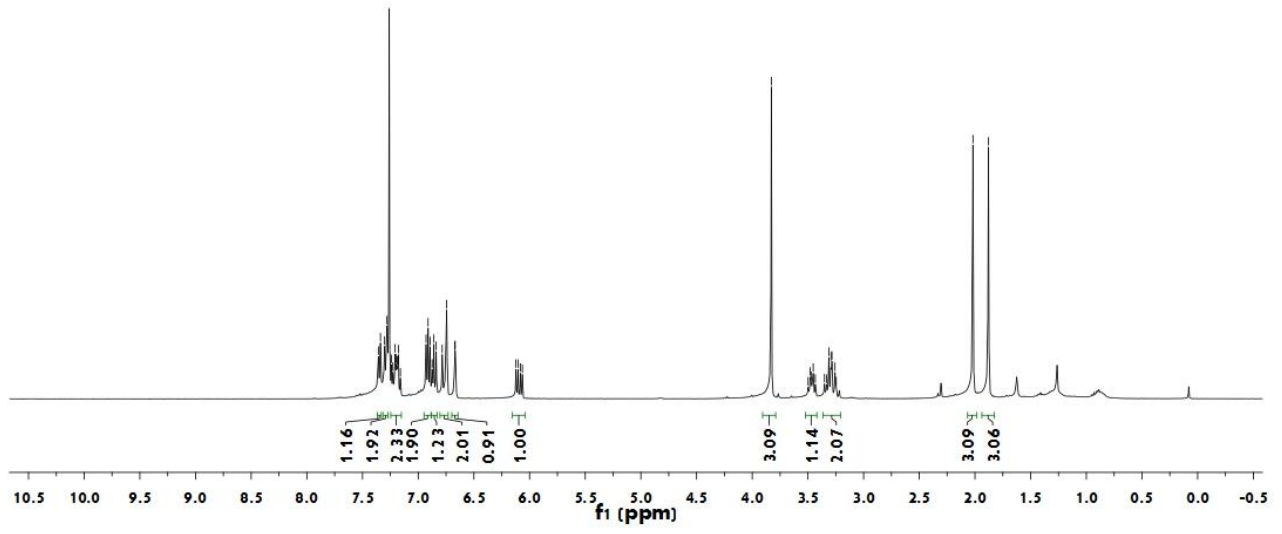

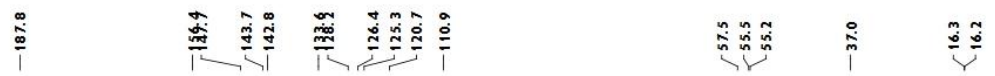

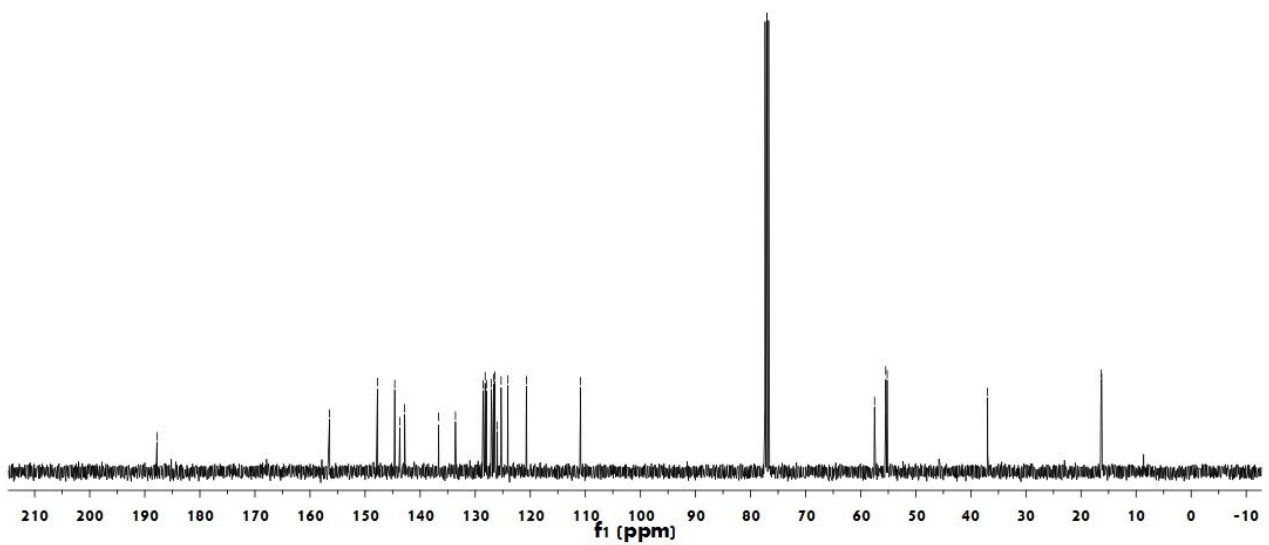




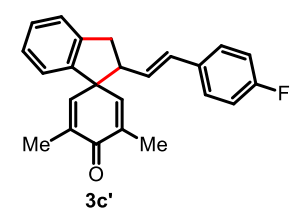

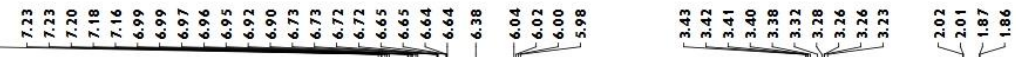
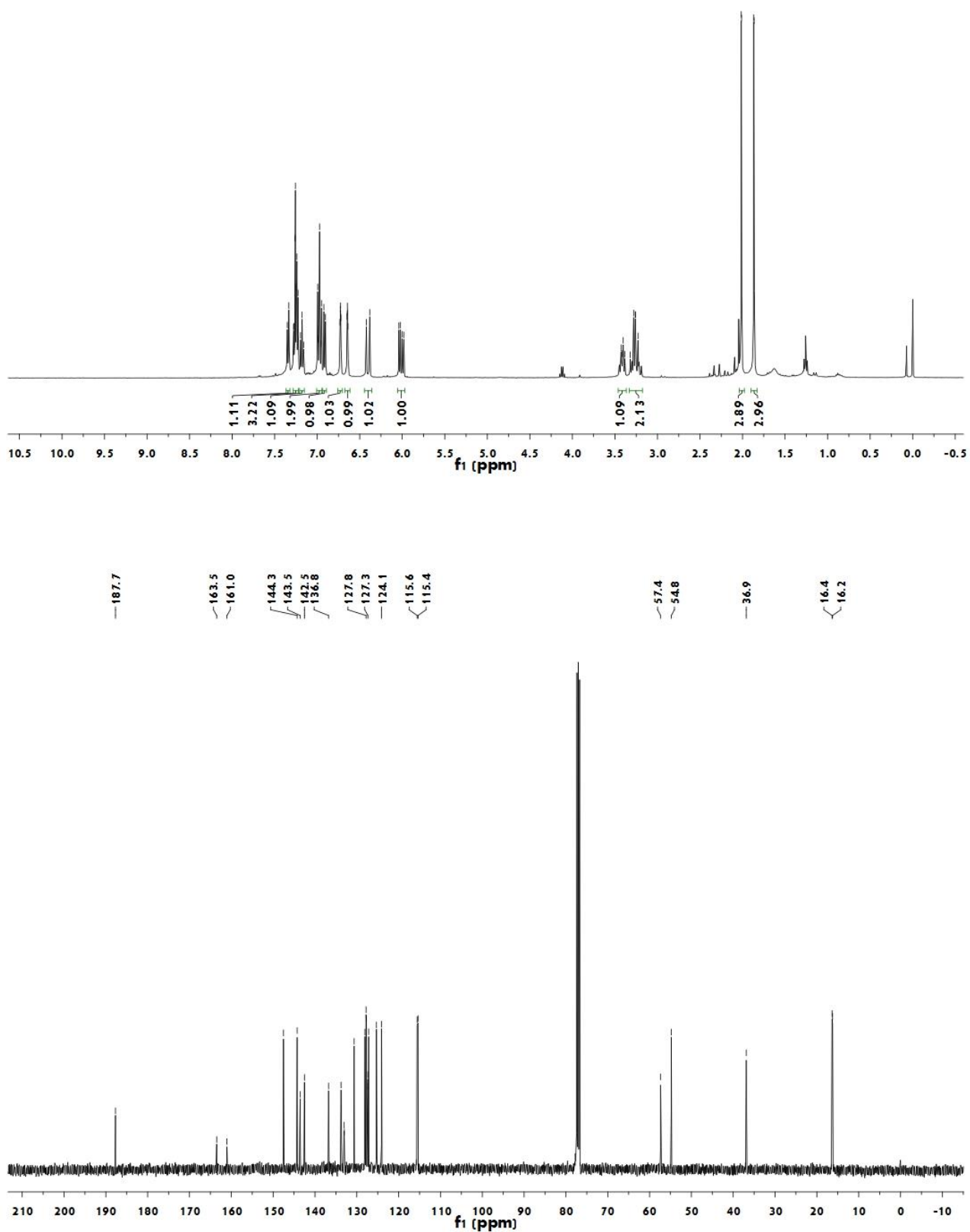

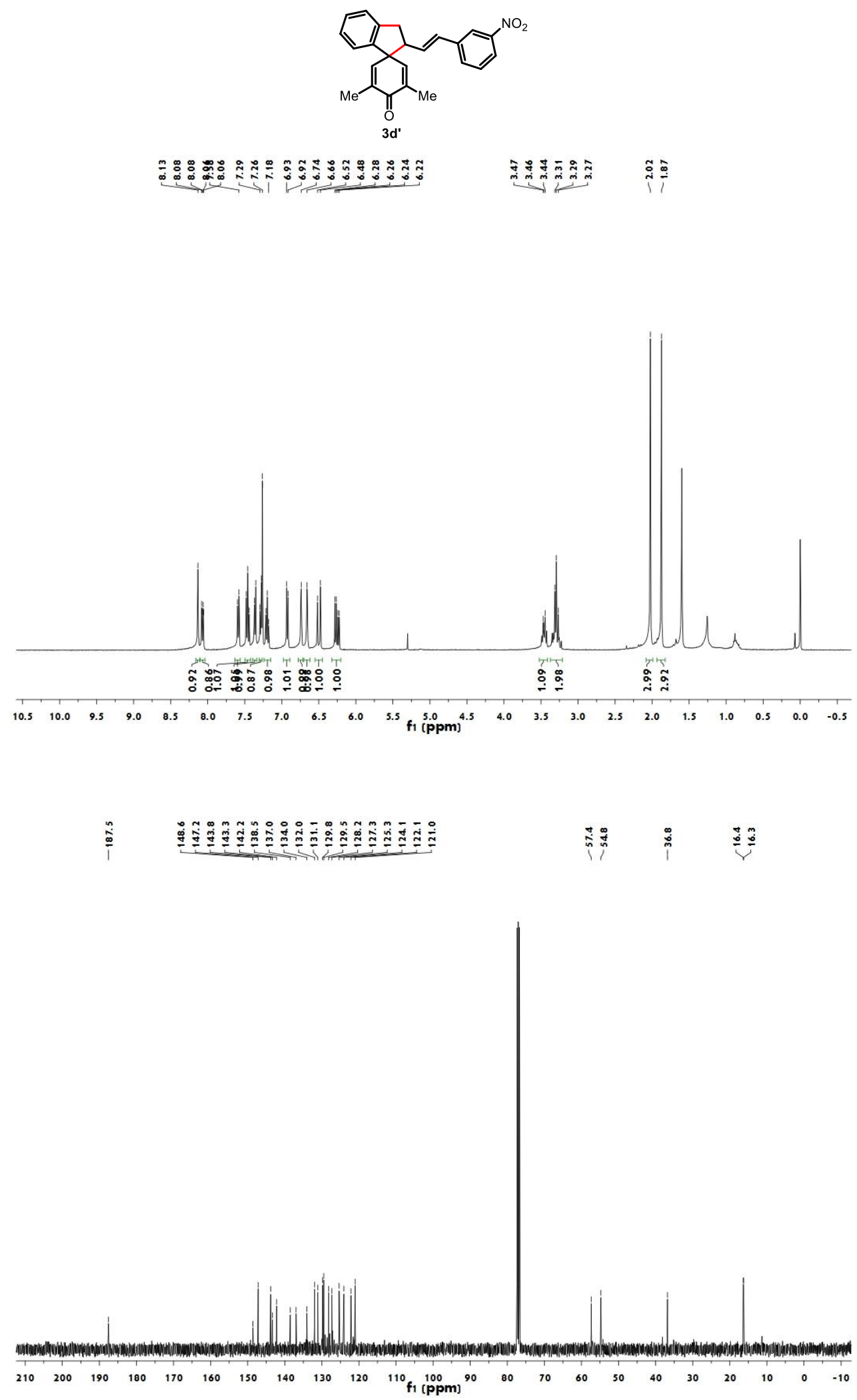

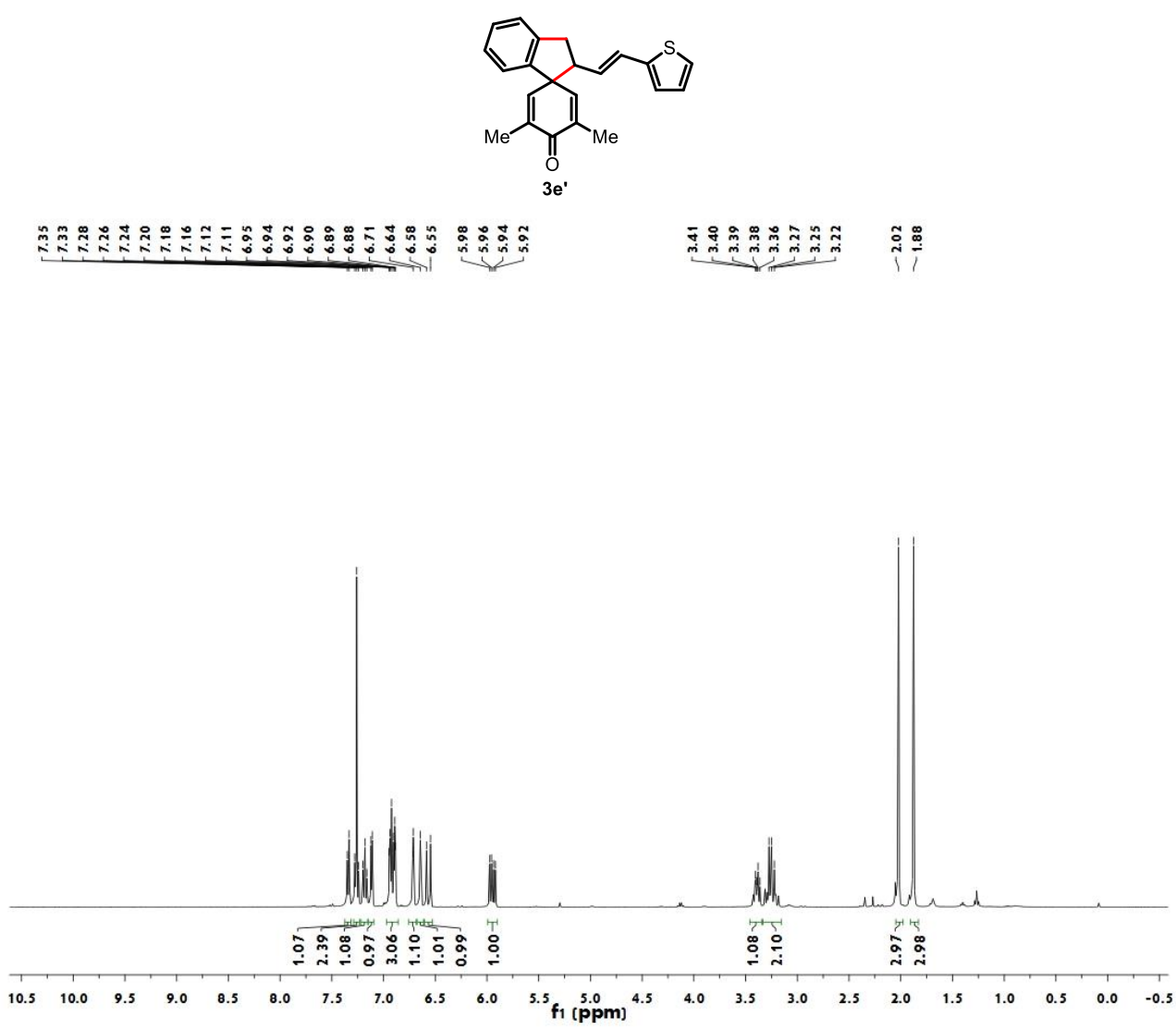

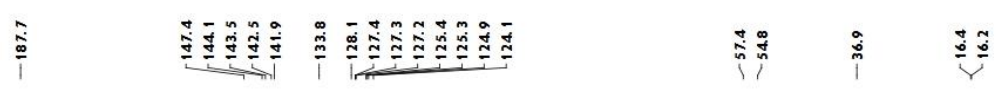

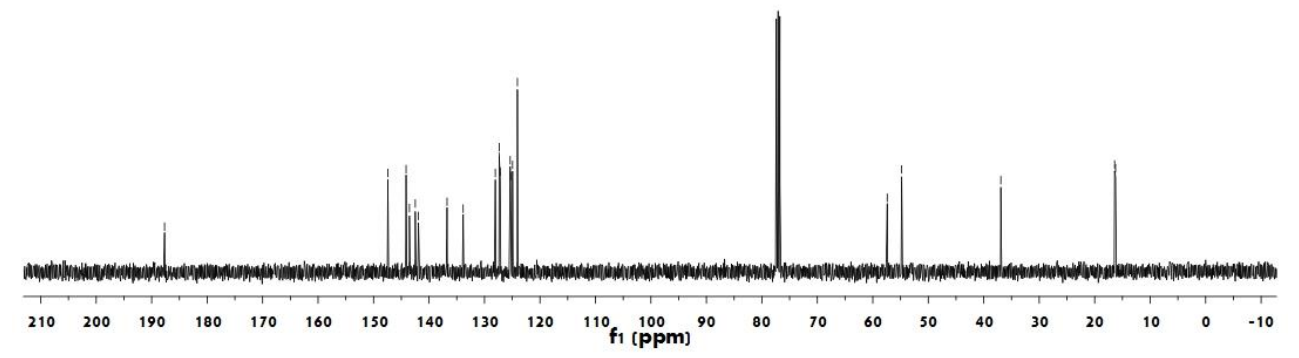




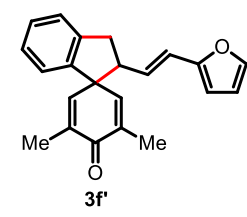

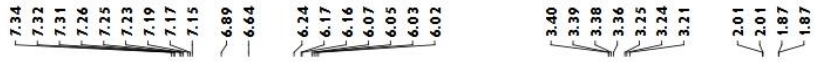
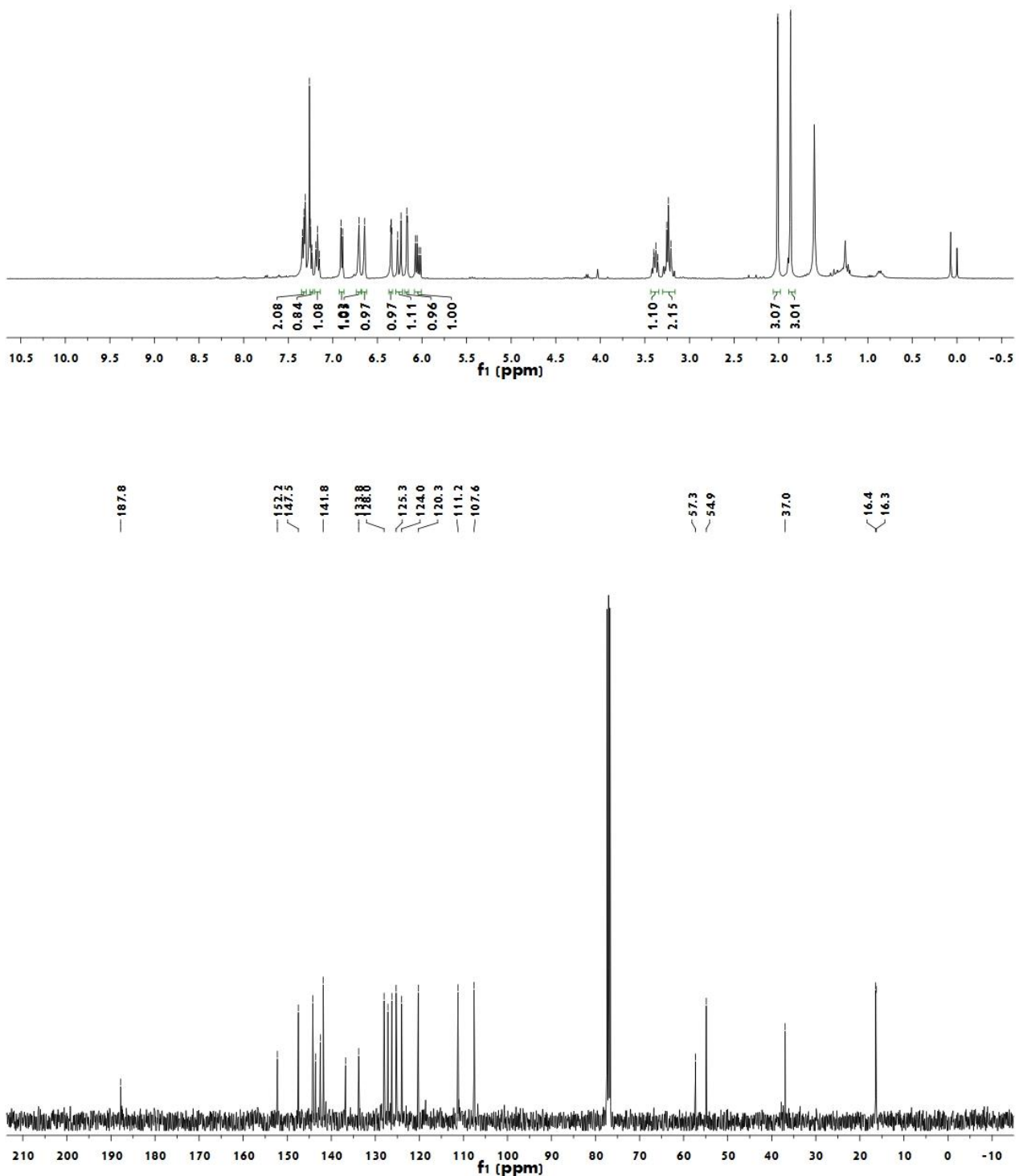

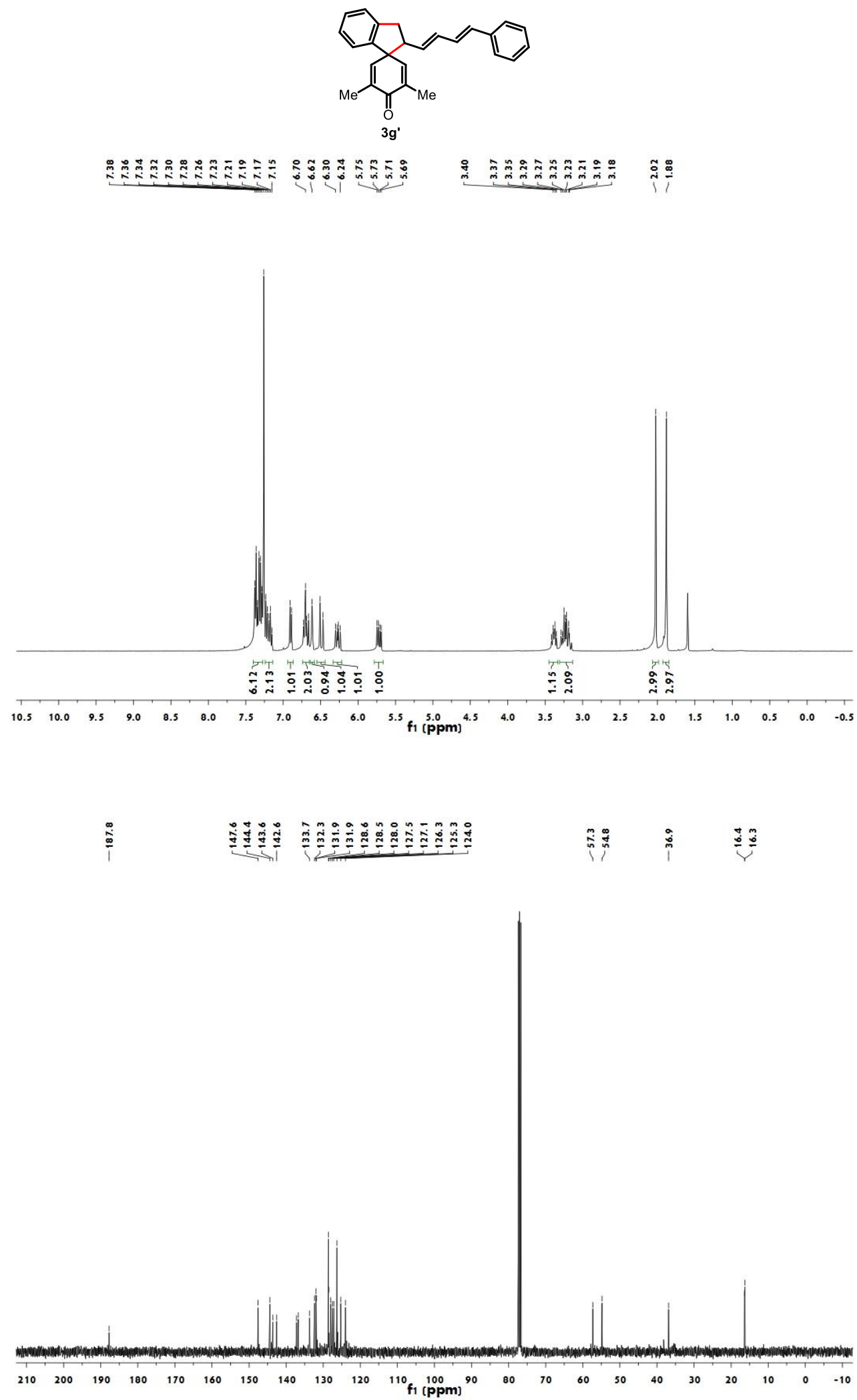